\title{
Finite-type invariants of w-knotted objects, I: w-knots and the Alexander polynomial
}

\author{
DROR BAR-NATAN \\ ZSUZSANNA DANCSO
}

This is the first in a series of papers studying w-knots, and more generally, w-knotted objects (w-braids, w-tangles, etc). These are classes of knotted objects which are wider, but weaker than their "úsual" counterparts.

The group of w-braids was studied (under the name "welded braids") by Fenn, Rimanyi and Rourke and was shown to be isomorphic to the McCool group of "basisconjugating" automorphisms of a free group $F_{n}$ : the smallest subgroup of $\operatorname{Aut}\left(F_{n}\right)$ that contains both braids and permutations. Brendle and Hatcher, in work that traces back to Goldsmith, have shown this group to be a group of movies of flying rings in $\mathbb{R}^{3}$. Satoh studied several classes of w-knotted objects (under the name "weaklyvirtual") and has shown them to be closely related to certain classes of knotted surfaces in $\mathbb{R}^{4}$. So w-knotted objects are algebraically and topologically interesting. Here we study finite-type invariants of w-braids and w-knots. Following Berceanu and Papadima, we construct homomorphic universal finite-type invariants of w-braids. The universal finite-type invariant of w-knots is essentially the Alexander polynomial. Much as the spaces $\mathcal{A}$ of chord diagrams for ordinary knotted objects are related to metrized Lie algebras, the spaces $\mathcal{A}^{w}$ of "arrow diagrams" for w-knotted objects are related to not-necessarily-metrized Lie algebras. Many questions concerning w-knotted objects turn out to be equivalent to questions about Lie algebras. Later in this paper series we re-interpret the work of Alekseev and Torossian on Drinfel'd associators and the Kashiwara-Vergne problem as a study of w-knotted trivalent graphs.

$57 \mathrm{M} 25,57 \mathrm{Q} 45$

\section{Introduction}

\subsection{Dreams}

We have a dream, ${ }^{1}$ at least partially founded on reality, that many of the difficult algebraic equations in mathematics, especially those that are written in graded spaces,

\footnotetext{
${ }^{1}$ Understanding the authors' history and psychology ought never be necessary to understand their papers, yet it may be helpful. Nothing material in the rest of this paper relies on Section 1.1.
} 
more especially those that are related in one way or another to quantum groups, and even more especially those related to the work of Etingof and Kazhdan [38], can be understood, and indeed, would appear more natural, in terms of finite-type invariants of various topological objects.

We believe this is the case for Drinfel'd's theory of associators [34], which can be interpreted as a theory of well-behaved universal finite-type invariants of parenthesized tangles $^{2}$ (see Le and Murakami [63], Bar-Natan [10]), and as a theory of universal finite-type invariants of knotted trivalent graphs (see Dancso [32]).

We believe this is the case for Drinfel'd's "Grothendieck-Teichmüller group" [35], which is better understood as a group of automorphisms of a certain algebraic structure, also related to universal finite-type invariants of parenthesized tangles (see BarNatan [11]).

And we're optimistic, indeed we believe, that sooner or later the work of Etingof and Kazhdan [38] on quantization of Lie bialgebras will be re-interpreted as a construction of a well-behaved universal finite-type invariant of virtual knots (see Kauffman [54]) or of some other class of virtually knotted objects. Some steps in that direction were taken by Haviv [49].

We have another dream, to construct a useful "algebraic knot theory". As at least a partial writeup exists (see Bar-Natan [12]), we'll only state that an important ingredient necessary to fulfil that dream would be a "closed form" 3 formula for an associator, at least in some reduced sense. Formulae for associators or reduced associators were in themselves the goal of several studies undertaken for various other reasons (see Le and Murakami [62], Lieberum [71], Kurlin [60] and Lee [65]).

\subsection{Stories}

Thus, the first named author, DBN, was absolutely delighted when in January 2008 Anton Alekseev described to him his joint work [2] with Charles Torossian: Anton told DBN that they found a relationship between the Kashiwara-Vergne conjecture [52], a cousin of the Duflo isomorphism (which DBN already knew to be knot-theoretic [21]), and associators taking values in a space called $\mathfrak{s d e r}$, which he could identify as "treelevel Jacobi diagrams", also a knot-theoretic space related to the Milnor invariants (see Bar-Natan [7], Habegger and Masbaum [45]). What's more, Anton told DBN that in certain quotient spaces the Kashiwara-Vergne conjecture can be solved explicitly; this should lead to some explicit associators!

\footnotetext{
2" q-tangles" in Le and Murakami [63], "non-associative tangles" in Bar-Natan [10].

${ }^{3}$ The phrase "closed form" in itself requires an explanation. See Section 4.2.
} 
So DBN spent the following several months trying to understand [2], which eventually led to this sequence of papers. One main thing we learned is that the Alekseev-Torossian paper, and with it the Kashiwara-Vergne (KV) conjecture, fit very nicely with our first dream described above, about interpreting algebra in terms of knot theory. Indeed much of [2] can be reformulated as a construction and a discussion of a well-behaved universal finite-type invariant ${ }^{4} Z$ of a certain class of knotted objects (which we will call w-knotted), a certain natural quotient of the space of virtual knots more precisely, virtual trivalent tangles); this will be the subject of the second paper in the series. It is also possible to provide a topological interpretation (and independent topological proof) of the formula of Alekseev, Enriquez and Torossian [1] for explicit solutions to the KV problem in terms of associators. This will be done in the third paper. And our hopes remain high that later we (or somebody else) will be able to exploit this relationship in directions compatible with our second dream described above, on the construction of an "algebraic knot theory".

The story, in fact, is prettier than we were hoping for, as it has the following additional qualities:

- w-knotted objects are quite interesting in themselves: as stated in the abstract, they are related to combinatorial group theory via "basis-conjugating" automorphisms of a free group $F_{n}$, to groups of movies of flying rings in $\mathbb{R}^{3}$, and more generally, to certain classes of knotted surfaces in $\mathbb{R}^{4}$. The references include Goldsmith [40], McCool [74], Fenn, Rimányi and Rourke [39], Satoh [81], and Brendle and Hatcher [28].

- The "chord diagrams" for w-knotted objects (really, these are "arrow diagrams") describe formulae for invariant tensors in spaces pertaining to not-necessarilymetrized Lie algebras in much the same way as ordinary chord diagrams for ordinary knotted objects describe formulae for invariant tensors in spaces pertaining to metrized Lie algebras. This observation is bound to have further implications.

- Arrow diagrams also describe the Feynman diagrams of topological BF theory (see Cattaneo, Cotta-Ramusino, Fröhlich and Martellini [31; 30]) and of a certain class of Chern-Simons theories (see Naot [76]). Thus, it is likely that our story is directly related to quantum field theory. ${ }^{5}$

\footnotetext{
${ }^{4}$ The notation $Z$ for universal finite-type invarants comes from the famous universal finite-type invariant of classical links, the Kontsevich integral.

${ }^{5}$ Some non-perturbative relations between BF theory and w-knots was discussed by Baez, Wise and Crans [5].
} 
- The main objective of this paper is to prove that when composed with the map from knots to w-knots, $Z$ becomes the Alexander polynomial. For links, it becomes an invariant stronger than the multi-variable Alexander polynomial, which contains the multi-variable Alexander polynomial as an easily identifiable reduction.

- On other w-knotted objects $Z$ has easily identifiable reductions that can be considered as "Alexander polynomials" with good behaviour relative to various knot-theoretic operations: cablings, compositions of tangles, etc. There is also a certain specific reduction of $Z$ that can be considered as an "ultimate Alexander polynomial"; in the appropriate sense, it is the minimal extension of the Alexander polynomial to other knotted objects which is well behaved under a whole slew of knot theoretic operations, including the ones named above. See Bar-Natan and Selmani [22], Bar-Natan [14].

- The true value of w-knots, though, is likely to emerge later, for we expect them to serve as a warmup example for what we expect will be even more interesting: the study of virtual knots, or v-knots. We expect v-knotted objects to provide the global context whose associated graded structure will be the Etingof-Kazhdan theory of deformation quantization of Lie bialgebras [38].

\subsection{The bigger picture}

Parallel to the w-story run the possibly more significant u-story and v-story. The u-story is about u-knots, or more generally, u-knotted objects (braids, links, tangles, etc), where " $u$ " stands for usual; hence the u-story is about classical knot theory. The v-story is about v-knots, or more generally, v-knotted objects, where "v" stands for virtual, in the sense of Kauffman [54].

The stories of u-, v- and w-knotted objects are quite different from each other. Yet they can be told along similar lines: first the knots (topology), then their finite-type invariants and their "chord diagrams" (combinatorics), then those map into certain universal enveloping algebras and similar spaces associated with various classes of Lie algebras (low algebra), and finally, in order to construct a "good" universal finite-type invariant, in each case one has to confront a certain deeper algebraic subject (high algebra). These stories are summarized in table form in Figure 1.

u-Knots map into v-knots, and v-knots map into w-knots. ${ }^{6}$ The other parts of our stories, the "combinatorics" and "low algebra" and "high algebra" rows of Figure 1,

\footnotetext{
${ }^{6}$ Though the composition " $u \rightarrow v \rightarrow w$ " is not 0 . In fact, the composed map $u \rightarrow w$ is injective. u-Knots, for example, are determined by the fundamental groups of their complements plus "peripheral systems" (or alternatively, by their "quandles" as in Joyce [50]), and this information is easily recovered from the w-knot images of u-knots. Similar considerations apply to other classes of u-knotted objects.
} 


\begin{tabular}{|c|c|c|c|}
\hline & u-knots & v-knots & w-knots \\
\hline $\begin{array}{l}\text { कृ. } \\
\frac{0}{0} \\
\frac{0}{0}\end{array}$ & $\begin{array}{l}\text { Ordinary (usual) knotted } \\
\text { objects in 3D: braids, } \\
\text { knots, links, tangles, } \\
\text { knotted graphs, etc. }\end{array}$ & $\begin{array}{l}\text { Virtual knotted objects: } \\
\text { "algebraic" knotted } \\
\text { objects, or "not } \\
\text { specifically embedded" } \\
\text { knotted objects; knots } \\
\text { drawn on a surface, } \\
\text { modulo stabilization. }\end{array}$ & $\begin{array}{l}\text { Ribbon knotted objects } \\
\text { in 4D; "flying rings". } \\
\text { Like v, but also with } \\
\text { "overcrossings } \\
\text { commute". }\end{array}$ \\
\hline 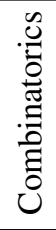 & $\begin{array}{l}\text { Chord diagrams and } \\
\text { Jacobi diagrams, modulo } \\
4 T, \text { STU, IHX, etc. }\end{array}$ & $\begin{array}{l}\text { Arrow diagrams and } \\
\text { v-Jacobi diagrams, } \\
\text { modulo } 6 T \text { and various } \\
\text { "directed" STUs and } \\
\text { IHXs, etc. }\end{array}$ & $\begin{array}{l}\text { Like v, but also with } \\
\text { "tails commute". Only } \\
\text { "two in one out" internal } \\
\text { vertices. }\end{array}$ \\
\hline 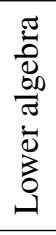 & $\begin{array}{l}\text { Finite-dimensional } \\
\text { metrized Lie algebras, } \\
\text { representations, and } \\
\text { associated spaces. }\end{array}$ & $\begin{array}{l}\text { Finite-dimensional Lie } \\
\text { bialgebras, } \\
\text { representations, and } \\
\text { associated spaces. }\end{array}$ & $\begin{array}{l}\text { Finite-dimensional } \\
\text { co-commutative Lie } \\
\text { bialgebras (ie } \mathfrak{g} \ltimes \mathfrak{g}^{*} \text { ), } \\
\text { representations, and } \\
\text { associated spaces. } \\
\end{array}$ \\
\hline 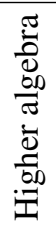 & $\begin{array}{l}\text { The Drinfel'd theory of } \\
\text { associators. }\end{array}$ & $\begin{array}{l}\text { Likely, quantum groups } \\
\text { and the Etingof-Kazhdan } \\
\text { theory of quantization of } \\
\text { Lie bialgebras. }\end{array}$ & $\begin{array}{l}\text { The Kashiwara-Vergne- } \\
\text { Alekseev-Torossian } \\
\text { theory of convolutions } \\
\text { on Lie groups and Lie } \\
\text { algebras. }\end{array}$ \\
\hline
\end{tabular}

Figure 1: The u-v-w stories

are likewise related, and this relationship is a crucial part of our overall theme. Thus, we cannot and will not tell the w-story in isolation, and while it is central to this article, we will necessarily also include some episodes from the $\mathrm{u}$ and $\mathrm{v}$ series.

\subsection{Plans}

In this paper we study w-braids and w-knots; the main result is Theorem 3.26, which states that the universal finite-type invariant of w-knots is essentially the Alexander polynomial. However, starting with braids and taking a classical approach to finite-type invariants, this paper also serves as a gentle introduction to the subsequent papers and in particular to [16], where we will present a more algebraic point of view. For more detailed information on the content consult the first summary paragraphs at the beginning of each section or here below. An "Odds and ends" section follows the main sections. 
Section 2, w-braids This section is largely a compilation of existing literature, though we also introduce the language of arrow diagrams that we use throughout the rest of the paper. In Sections 2.1 and 2.2 we define v-braids and then w-braids and survey their relationship with basis-conjugating automorphisms of free groups and with "the group of (horizontal) flying rings in $\mathbb{R}^{3}$ " (really, a group of knotted tubes in $\mathbb{R}^{4}$ ). In Section 2.3 we play the usual game of introducing finite-type invariants, weight systems, chord diagrams (arrow diagrams, for this case), and $4 T$-like relations. In Section 2.4 we define and construct a universal finite-type invariant $Z$ for w-braids; it turns out that the only algebraic tool we need to use is the formal exponential function $\exp (a):=\sum a^{n} / n !$. In Section 2.5 we study some good algebraic properties of $Z$, its injectivity, and its uniqueness, and we conclude with the slight modifications needed for the study of non-horizontal flying rings.

Section 3, w-knots In Section 3.1 we define v-knots and w-knots (long v-knots and long w-knots, to be precise) and discuss a map $v \rightarrow w$. In Section 3.2 we determine the space of "chord diagrams" for w-knots to be the space $\mathcal{A}^{w}(\uparrow)$ of arrow diagrams modulo $\overrightarrow{4 T}$ and TC relations, and in Section 4.1 we compute some relevant dimensions. In Section 3.4 we show that $\mathcal{A}^{w}(\uparrow)$ can be re-interpreted as a space of trivalent graphs modulo STU- and IHX-like relations, and is therefore related to Lie algebras (Section 3.5). This allows us to completely determine $\mathcal{A}^{w}(\uparrow)$. With no difficulty in Section 3.3 we construct a universal finite-type invariant for w-knots. With a bit of further difficulty we show in Section 3.6 that it is essentially equal to the Alexander polynomial.

Acknowledgements We wish to thank the anonymous referee, Anton Alekseev, Jana Archibald, Scott Carter, Karene Chu, Iva Halacheva, Joel Kamnitzer, Lou Kauffman, Peter Lee, Louis Leung, Jean-Baptiste Meilhan, Dylan Thurston, Daniel Tubbenhauer and Lucy Zhang for comments and suggestions.

This work was partially supported by NSERC grant RGPIN 262178. See [15] for electronic version, videos (wClips) and related files.

\section{2 w-braids}

This section is largely a compilation of existing literature, though we also introduce the language of arrow diagrams that we use throughout the rest of the paper. In Sections 2.1 and 2.2 we define v-braids and then w-braids and survey their relationship with basisconjugating automorphisms of free groups and with "the group of (horizontal) flying

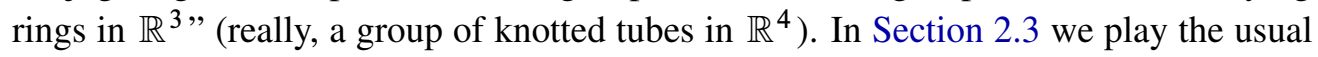
game of introducing finite-type invariants, weight systems, chord diagrams (arrow 
diagrams, for this case), and 4T-like relations. In Section 2.4 we define and construct a universal finite-type invariant $Z$ for w-braids; it turns out that the only algebraic tool we need to use is the formal exponential function $\exp (a):=\sum a^{n} / n !$. In Section 2.5 we study some good algebraic properties of $Z$, its injectivity, and its uniqueness, and we conclude with the slight modifications needed for the study of non-horizontal flying rings.

\subsection{Preliminary: virtual braids, or v-braids}

Our main object of study for this section, w-braids, are best viewed as "virtual braids" $[24 ; 55 ; 25]$, or v-braids, modulo one additional relation; hence, we start with v-braids.

It is simplest to define v-braids in terms of generators and relations, either algebraically or pictorially. This can be done in at least two ways: the easier-at-first but philosophically less satisfying "planar" way, and the harder-to-digest but morally more correct "abstract" way. ${ }^{7}$

2.1.1 The "planar" way For a natural number $n$ set $v B_{n}$ to be the group generated by symbols $\sigma_{i}(1 \leq i \leq n-1)$, called "crossings" and graphically represented by an overcrossing $\Upsilon$ "between strand $i$ and strand $i+1$ " (with inverse $\left.\lambda^{\top}\right),{ }^{8}$ and $s_{i}$, called "virtual crossings" and graphically represented by a non-crossing, $\chi$, also "between strand $i$ and strand $i+1$ ", subject to the following relations:

- The subgroup of $v B_{n}$ generated by the virtual crossings $s_{i}$ is the symmetric group $S_{n}$, and the $s_{i}$ correspond to the transpositions $(i, i+1)$. That is, we have

$$
s_{i}^{2}=1, \quad s_{i} s_{i+1} s_{i}=s_{i+1} s_{i} s_{i+1}, \quad \text { and } \quad \text { if }|i-j|>1, \text { then } s_{i} s_{j}=s_{j} s_{i} .
$$

In pictures, this is
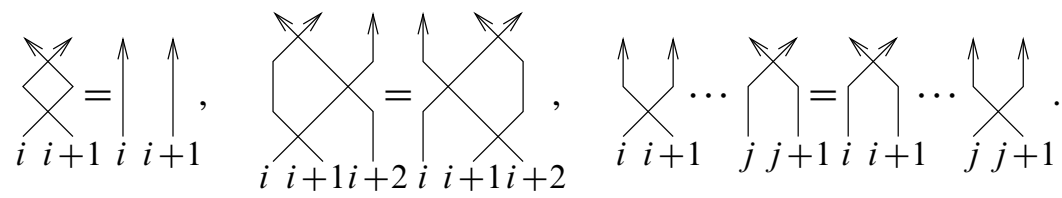

${ }^{7}$ Compare with a similar choice that exists in the definition of manifolds, as either appropriate subsets of some ambient Euclidean spaces (modulo some equivalences) or as abstract gluings of coordinate patches (modulo some other equivalences). Here in the "planar" approach of Section 2.1.1 we consider v-braids as "planar" objects, and in the "abstract approach" of Section 2.1.2 they are just "gluings" of abstract "crossings", not drawn anywhere in particular.

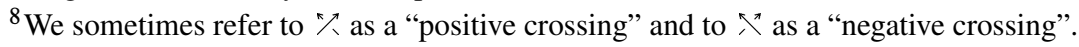


Note that we read our braids from bottom to top, and that all relations (and most pictures in this paper) are local: the braids may be bigger than shown but the parts not shown remain the same throughout a relation.

- The subgroup of $v B_{n}$ generated by the crossings $\sigma_{i}$ is the usual braid group $u B_{n}$, and $\sigma_{i}$ corresponds to the "braiding of strand $i$ over strand $i+1$ ". That is, we have

$$
\sigma_{i} \sigma_{i+1} \sigma_{i}=\sigma_{i+1} \sigma_{i} \sigma_{i+1}, \quad \text { and } \quad \text { if }|i-j|>1 \text { then } \sigma_{i} \sigma_{j}=\sigma_{j} \sigma_{i} \text {. }
$$

In pictures, dropping the indices, this is

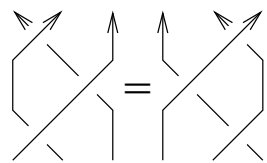

and

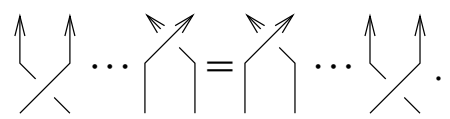

The first of these relations is the "Reidemeister 3 move" 9 of knot theory. The second is sometimes called "locality in space" [10].

- Some "mixed relations", that is,

$$
s_{i} \sigma_{i+1}^{ \pm 1} s_{i}=s_{i+1} \sigma_{i}^{ \pm 1} s_{i+1}, \quad \text { and } \quad \text { if }|i-j|>1 \text {, then } s_{i} \sigma_{j}=\sigma_{j} s_{i} .
$$

In pictures, this is
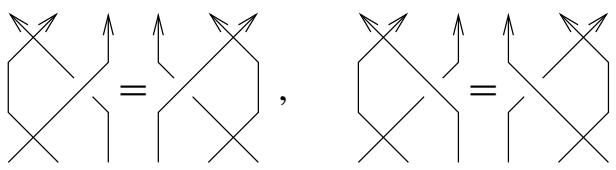

and

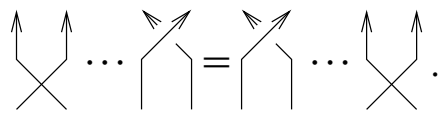

Remark 2.1 The "skeleton" of a v-braid $B$ is the set of strands appearing in it, retaining the association between their beginning and ends but ignoring all the crossing information. More precisely, it is the permutation induced by tracing along $B$, and even more precisely it is the image of $B$ via the "skeleton morphism" $\varsigma: v B_{n} \rightarrow S_{n}$ defined by $\varsigma\left(\sigma_{i}\right)=\varsigma\left(s_{i}\right)=s_{i}$ (or pictorially, by $\left.\varsigma(\%)=\varsigma(X)=\chi\right)$. Thus, the symmetric group $S_{n}$ is both a subgroup and a quotient group of $v B_{n}$.

Just as there are pure braids to accompany braids, there are pure virtual braids as well:

Definition 2.2 A pure v-braid is a v-braid whose skeleton is the identity permutation. The group $P v B_{n}$ of all pure v-braids is simply the kernel of the skeleton morphism ऽ:v $B_{n} \rightarrow S_{n}$.

\footnotetext{
${ }^{9}$ The Reidemeister 2 move is the relation $\sigma_{i} \sigma_{i}^{-1}=1$ which is part of the definition of a group. There is no Reidemeister 1 move in the theory of braids.
} 
We note the short exact sequence of group homomorphisms

$$
1 \longrightarrow P v B_{n} \hookrightarrow v B_{n} \stackrel{5}{\longrightarrow} S_{n} \longrightarrow 1 .
$$

This short exact sequence splits, with the splitting given by the inclusion $S_{n} \hookrightarrow v B_{n}$ mentioned above (1). Therefore, we have that

$$
v B_{n}=P v B_{n} \rtimes S_{n} .
$$

2.1.2 The "abstract" way The relations (2) and (6) that govern the behaviour of virtual crossings say precisely that virtual crossings really are "virtual": if a piece of strand is routed within a braid so that there are only virtual crossings around it, it can be rerouted in any other "virtual only" way, provided the ends remain fixed (this is Kauffman's "detour move" $[54 ; 55])$. Since a v-braid $B$ is independent of the routing of virtual pieces of strand, we may as well never supply this routing information.

Thus, for example, a perfectly fair verbal description of the following (pure!) v-braid is "strand 1 goes over strand 3 by a positive crossing then positively over strand 2 then negatively over 3 , then 2 goes positively over 1 ".

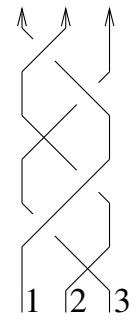

We don't need to specify how strand 1 got to be near strand 3 so that it can go over it; it got there by means of virtual crossings, and it doesn't matter how. Hence we arrive at the following "abstract" presentation of $P v B_{n}$ and $v B_{n}$.

Proposition 2.3 [24, Theorems 1 and 2] (1) The group $P v B_{n}$ of pure $v$-braids is isomorphic to the group generated by symbols $\sigma_{i j}$ for $1 \leq i \neq j \leq n$ (meaning "strand $i$ crosses over strand $j$ at a positive crossing"), ${ }^{10}$ subject to the third Reidemeister move and to locality in space (compare with (3) and (4)):

$$
\begin{cases}\sigma_{i j} \sigma_{i k} \sigma_{j k}=\sigma_{j k} \sigma_{i k} \sigma_{i j} & \text { whenever }|\{i, j, k\}|=3, \\ \sigma_{i j} \sigma_{k l}=\sigma_{k l} \sigma_{i j} & \text { whenever }|\{i, j, k, l\}|=4 .\end{cases}
$$

(2) If $\tau \in S_{n}$, then with the action $\sigma_{i j}^{\tau}:=\sigma_{\tau i, \tau j}$ we recover the semi-direct product decomposition $v B_{n}=P v B_{n} \rtimes S_{n}$.

${ }^{10}$ The inverse, $\sigma_{i j}^{-1}$, is "strand $i$ crosses over strand $j$ at a negative crossing". 


\subsection{On to w-braids}

To define w-braids, we break the symmetry between overcrossings and undercrossings by imposing one of the "forbidden moves" in virtual knot theory, but not the other:

$$
\sigma_{i} \sigma_{i+1} s_{i}=s_{i+1} \sigma_{i} \sigma_{i+1}, \quad \text { yet } s_{i} \sigma_{i+1} \sigma_{i} \neq \sigma_{i+1} \sigma_{i} s_{i+1} .
$$

Alternatively,

$$
\sigma_{i j} \sigma_{i k}=\sigma_{i k} \sigma_{i j}, \quad \text { yet } \quad \sigma_{i k} \sigma_{j k} \neq \sigma_{j k} \sigma_{i k}
$$

In pictures, this is

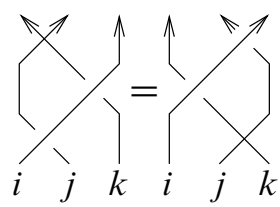

yet

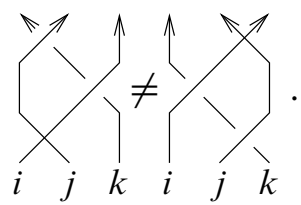

The relation we have just imposed may be called the "unforbidden relation", or, perhaps more appropriately, the "overcrossings commute" relation, abbreviated OC. Ignoring the non-crossings ${ }^{11} X$, the OC relation says that it is the same if strand $i$ first crosses over strand $j$ and then over strand $k$, or if it first crosses over strand $k$ and then over strand $j$. The "undercrossings commute" relation UC, the one we do not impose in (9), would say the same except with "under" replacing "over".

Definition 2.4 The group of w-braids is $w B_{n}:=v B_{n} /$ OC. Note that $\varsigma$ descends to $w B_{n}$, and hence we can define the group $P w B_{n}$ of pure w-braids to be the kernel of the map $\varsigma: w B_{n} \rightarrow S_{n}$. We still have a split exact sequence as in (7) and a thus, a semi-direct product decomposition $w B_{n}=P w B_{n} \rtimes S_{n}$.

Exercise 2.5 Show that the OC relation is equivalent to the relation

$$
\sigma_{i}^{-1} s_{i+1} \sigma_{i}=\sigma_{i+1} s_{i} \sigma_{i+1}^{-1}, \quad \text { or }
$$

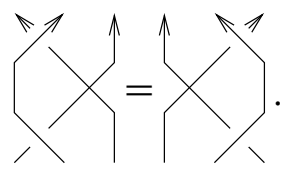

While for most of this paper the pictorial/algebraic definition of w-braids (and other w-knotted objects) will suffice, we ought to describe at least briefly a few further interpretations of $w B_{n}$.

${ }^{11}$ Why this is appropriate was explained in the previous section. 
2.2.1 The group of flying rings Let $X_{n}$ be the space of all placements of $n$ numbered disjoint geometric circles in $\mathbb{R}^{3}$ such that all circles are parallel to the $x y$ plane. Such placements will be called horizontal. ${ }^{12}$ A horizontal placement is determined by the centres in $\mathbb{R}^{3}$ of the $n$ circles and by $n$ radii, so $\operatorname{dim} X_{n}=3 n+n=4 n$. The permutation group $S_{n}$ acts on $X_{n}$ by permuting the circles, and one may think of the quotient $\widetilde{X}_{n}:=X_{n} / S_{n}$ as the space of all horizontal placements of $n$ unmarked circles in $\mathbb{R}^{3}$. The fundamental group $\pi_{1}\left(\tilde{X}_{n}\right)$ is a group of paths traced by $n$ disjoint horizontal circles (modulo homotopy), so it is fair to think of it as "the group of flying rings".

Theorem 2.6 The group of pure $w$-braids $P w B_{n}$ is isomorphic to the group of flying rings $\pi_{1}\left(X_{n}\right)$. The group $w B_{n}$ is isomorphic to the group of unmarked flying rings $\pi_{1}\left(\widetilde{X}_{n}\right)$.

For the proof of this theorem, see [40; 81] and especially [28, Proposition 3.3]. Here we will content ourselves with pictures describing the images of the generators of $w B_{n}$ in $\pi_{1}\left(\tilde{X}_{n}\right)$ and a few comments.
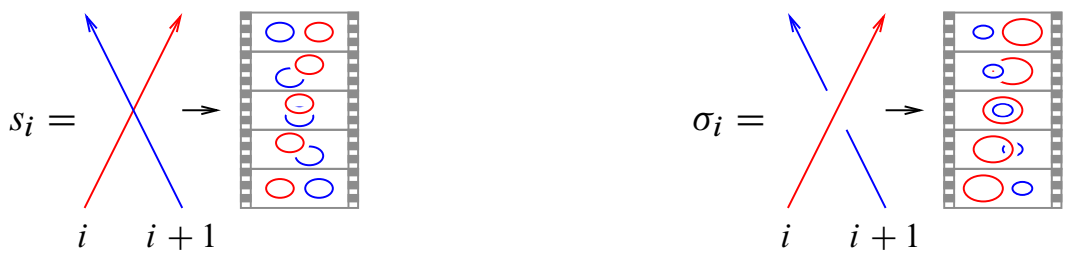

Thus, we map the permutation $s_{i}$ to the movie clip in which ring number $i$ trades places with ring number $i+1$ by having the two fly around each other. This acrobatic feat is performed in $\mathbb{R}^{3}$ and it does not matter if ring number $i$ goes "above" or "below" or "left" or "right" of ring number $i+1$ when they trade places, as all of these possibilities are homotopic. More interestingly, we map the braiding $\sigma_{i}$ to the movie clip in which ring $i+1$ shrinks a bit and flies through ring $i$. It is a worthwhile exercise for the reader to verify that the relations in the definition of $w B_{n}$ become homotopies of movie clips. Of these relations it is most interesting to see why the "overcrossings commute" relation $\sigma_{i} \sigma_{i+1} s_{i}=s_{i+1} \sigma_{i} \sigma_{i+1}$ holds, yet the "undercrossings commute" relation $\sigma_{i}^{-1} \sigma_{i+1}^{-1} s_{i}=s_{i+1} \sigma_{i}^{-1} \sigma_{i+1}^{-1}$ doesn't.

Exercise 2.7 To be perfectly precise, we have to specify the fly-through direction. In our notation, $\sigma_{i}$ means that the ring corresponding to the strand going under (in the local picture for $\sigma_{i}$ ) approaches from below the bigger ring representing the strand

\footnotetext{
${ }^{12}$ For the group of non-horizontal flying rings see Section 2.5.4.
} 
going over, then flies through it and exists above. For $\sigma_{i}^{-1}$ we are "playing the movie backwards", ie the ring of the strand going under comes from above and exits below the ring of the "over" strand.

Let "the signed $w$ braid group", $s w B_{n}$, be the group of horizontal flying rings where both fly-through directions are allowed. This introduces a "sign" for each crossing $\sigma_{i}$,
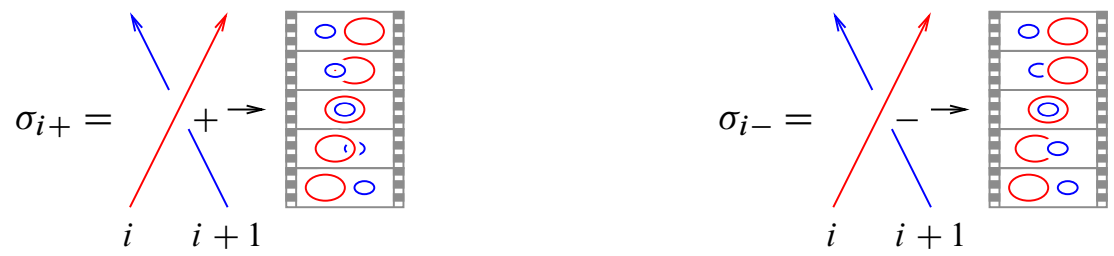

In other words, $s w B_{n}$ is generated by $s_{i}, \sigma_{i+}$ and $\sigma_{i-}$, for $i=1, \ldots, n-1$. Check that $\sigma_{i-}=s_{i} \sigma_{i+}^{-1} s_{i}$ in $s w B_{n}$, and this, along with the other obvious relations implies $s w B_{n} \cong w B_{n}$.

2.2.2 Certain ribbon tubes in $\mathbb{R}^{4}$ With time as the added dimension, a flying ring in $\mathbb{R}^{3}$ traces a tube (an annulus) in $\mathbb{R}^{4}$, as shown below:
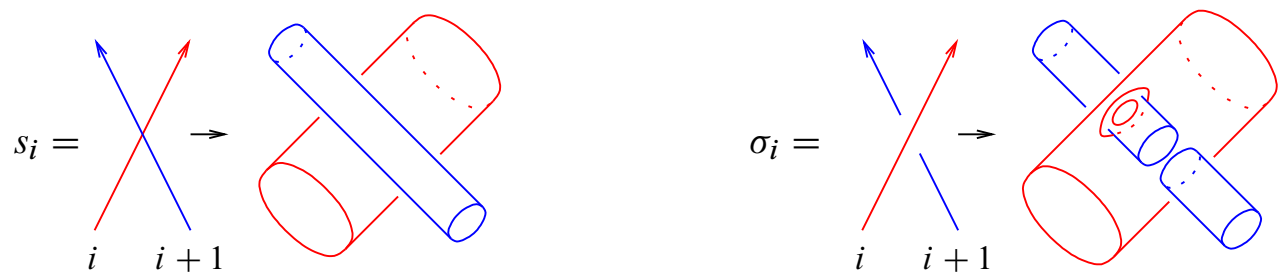

Note that we adopt here the drawing conventions of Carter and Saito [29]: we draw surfaces as if they were projected from $\mathbb{R}^{4}$ to $\mathbb{R}^{3}$, and we cut them open whenever they are "hidden" by something with a higher fourth coordinate.

Note also that the tubes we get in $\mathbb{R}^{4}$ always bound natural 3D "solids"; their "insides", in the pictures above. These solids are disjoint in the case of $s_{i}$ and have a very specific kind of intersection in the case of $\sigma_{i}$ : these are transverse intersections with no triple points, and their inverse images are a meridional disk on the "thin" solid tube and an interior disk on the "thick" one. By analogy with the case of ribbon knots and ribbon singularities in $\mathbb{R}^{3}$ (see eg [53, Chapter V]) and following Satoh [81], we call these kinds of intersections of solids in $\mathbb{R}^{4}$ "ribbon singularities" and thus, our tubes in $\mathbb{R}^{4}$ are always "ribbon tubes".

2.2.3 Basis conjugating automorphisms of $\boldsymbol{F}_{\boldsymbol{n}}$ Let $F_{n}$ be the free (non-abelian) group with generators $\xi_{1}, \ldots, \xi_{n}$. Artin's theorem [4, Theorems 15 and 16] says that 
the (usual) braid group $u B_{n}$ (equivalently, the subgroup of $w B_{n}$ generated by the $\sigma_{i}$ ) has a faithful right action on $F_{n}$. In other words, $u B_{n}$ is isomorphic to a subgroup $H$ of $\operatorname{Aut}^{\mathrm{op}}\left(F_{n}\right)$ (the group of automorphisms of $F_{n}$ with opposite multiplication, ie $\left.\psi_{1} \psi_{2}:=\psi_{2} \circ \psi_{1}\right)$. Precisely, using $(\xi, B) \mapsto \xi / / B$ to denote the right action of $\operatorname{Aut}^{\mathrm{op}}\left(F_{n}\right)$ on $F_{n}$, the subgroup $H$ consists of those automorphisms $B: F_{n} \rightarrow F_{n}$ of $F_{n}$ that satisfy the following two conditions:

(1) $B$ maps any generator $\xi_{i}$ to a conjugate of a generator (possibly different). That is, there is a permutation $\beta \in S_{n}$ and elements $a_{i} \in F_{n}$ such that for every $i$,

$$
\xi_{i} / / B=a_{i}^{-1} \xi_{\beta(i)} a_{i} .
$$

(2) $B$ fixes the ordered product of the generators of $F_{n}$,

$$
\xi_{1} \xi_{2} \cdots \xi_{n} / / B=\xi_{1} \xi_{2} \cdots \xi_{n} .
$$

McCool's theorem ${ }^{13}$ [74] says that almost the same statement holds true ${ }^{14}$ for the bigger group $w B_{n}$ : namely, $w B_{n}$ is isomorphic to the subgroup of $\operatorname{Aut}^{\mathrm{op}}\left(F_{n}\right)$ consisting of automorphisms satisfying only the first condition above. So $w B_{n}$ is precisely the group of "basis-conjugating" automorphisms of the free group $F_{n}$, the group of those automorphisms which map any "basis element" in $\left\{\xi_{1}, \ldots, \xi_{n}\right\}$ to a conjugate of a (possibly different) basis element.

The relevant action is explicitly defined on the generators of $w B_{n}$ and $F_{n}$ as follows (we state how each generator of $w B_{n}$ acts on each generator of $F_{n}$, in each case omitting the generators of $F_{n}$ which are fixed under the action):

$$
\begin{aligned}
\left(\xi_{i}, \xi_{i+1}\right) / / s_{i} & =\left(\xi_{i+1}, \xi_{i}\right), \\
\left(\xi_{i}, \xi_{i+1}\right) / / \sigma_{i} & =\left(\xi_{i+1}, \xi_{i+1} \xi_{i} \xi_{i+1}^{-1}\right), \\
\xi_{j} / / \sigma_{i j} & =\xi_{i} \xi_{j} \xi_{i}^{-1} .
\end{aligned}
$$

It is a worthwhile exercise to verify that // respects the relations in the definition of $w B_{n}$ and that the permutation $\beta$ in (11) is the skeleton $\varsigma(B)$.

There is a more conceptual description of // in terms of the structure of $w B_{n+1}$. Consider the inclusions

$$
w B_{n} \stackrel{\iota}{\hookrightarrow} w B_{n+1} \stackrel{i_{u}}{\longleftrightarrow} F_{n} .
$$

Here $\iota$ is the inclusion of $w B_{n}$ into $w B_{n+1}$ by adding an inert $(n+1)^{\text {st }}$ strand (it is injective as it has a well-defined one sided inverse: the deletion of the $(n+1)^{\text {st }}$ strand).

\footnotetext{
${ }^{13}$ Strictly speaking, the main theorem of [74] is about $P w B_{n}$, yet it can easily be restated for $w B_{n}$.

${ }^{14}$ Though see Warning 2.8.
} 
The inclusion $i_{u}$ of the free group $F_{n}$ into $w B_{n+1}$ is defined by $i_{u}\left(\xi_{i}\right):=\sigma_{i, n+1}$, depicted as follows:

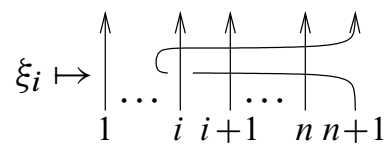

The image $i_{u}\left(F_{n}\right) \subset w B_{n+1}$ is the set of all w-braids whose first $n$ strands are straight and vertical, and whose $(n+1)^{\text {st }}$ strand wanders among the first $n$ strands mostly virtually (ie mostly using virtual crossings), occasionally slipping under one of those $n$ strands, but never going over anything. It is easier to see that this is indeed injective using the "flying rings" picture of Section 2.2.1. The image $i_{u}\left(F_{n}\right) \subset w B_{n+1}$ can be interpreted as the fundamental group of the complement in $\mathbb{R}^{3}$ of $n$ stationary rings (which is indeed $F_{n}$ ); in $i_{u}\left(F_{n}\right)$ the only ring in motion is the last, and it only goes under, or "through", other rings, so it can be replaced by a point object whose path is an element of the fundamental group. The injectivity of $i_{u}$ follows from this geometric picture.

One may explicitly verify that $i_{u}\left(F_{n}\right)$ is normalized by $\iota\left(w B_{n}\right)$ in $w B_{n+1}$ (that is, the set $i_{u}\left(F_{n}\right)$ is preserved by conjugation by elements of $\left.\iota\left(w B_{n}\right)\right)$. Thus, the following definition, pictured as

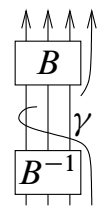

makes sense: for $B \in w B_{n} \subset w B_{n+1}$ and for $\gamma \in F_{n} \subset w B_{n+1}$,

$$
\gamma / / B:=i_{u}^{-1}\left(B^{-1} \gamma B\right)
$$

It is a worthwhile exercise to recover the explicit formulae in (12) from the above definition.

Warning 2.8 People familiar with the Artin story for ordinary braids should be warned that even though $w B_{n}$ acts on $F_{n}$ and the action is induced from the inclusions in (13) in much the same way as the Artin action is induced by inclusions

$$
u B_{n} \stackrel{\iota}{\hookrightarrow} u B_{n+1} \stackrel{i}{\hookleftarrow} F_{n},
$$

there are also some differences, and some further warnings apply:

- In the ordinary Artin story, $i\left(F_{n}\right)$ is the set of braids in $u B_{n+1}$ whose first $n$ strands are unbraided (that is, whose image in $u B_{n}$ via "dropping the last strand" 
is the identity). This is not true for w-braids. For w-braids, in $i_{u}\left(F_{n}\right)$ the last strand always goes "under" all other strands (or just virtually crosses them), but never "over".

- Thus, unlike the isomorphism $P u B_{n+1} \cong P u B_{n} \ltimes F_{n}$, it is not true that $P w B_{n+1}$ is isomorphic to $P w B_{n} \ltimes F_{n}$.

- The OC relation imposed in $w B$ breaks the symmetry between overcrossings and undercrossings. Thus, let $i_{o}: F_{n} \rightarrow w B_{n}$ be the "opposite" of $i_{u}$, mapping into braids in which the last strand is always "over" or virtual. Then $i_{o}$ is not injective (its image is in fact abelian) and its image is not normalized by $\iota\left(w B_{n}\right)$. So there is no "second" action of $w B_{n}$ on $F_{n}$ defined using $i_{o}$.

- For v-braids, both $i_{u}$ and $i_{o}$ are injective and there are two actions of $v B_{n}$ on $F_{n}$ : one defined by first projecting into w-braids, and the other defined by first projecting into v-braids modulo "undercrossings commute". Yet v-braids contain more information than these two actions can see. The "Kishino" v-braid below, for example, is visibly trivial if either overcrossings or undercrossings are made to commute, yet by computing its Kauffman bracket we know it is non-trivial as a v-braid [15, "The Kishino braid"]:

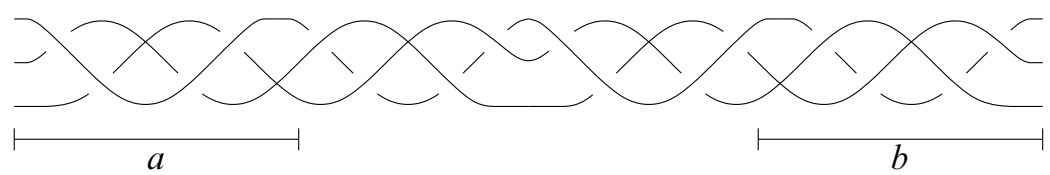

The commutator $a b^{-1} a^{-1} b$ of v-braids $a, b$ annihilated by OC/UC, respectively, with a minor cancellation.

Problem 2.9 Are $P v B_{n}$ and $P w B_{n}$ semi-direct products of free groups? For $P u B_{n}$, this is the well-known "combing of braids" and it follows from $P u B_{n} \cong P u B_{n-1} \ltimes F_{n-1}$ and induction.

Remark 2.10 Note that Gutiérrez and Krstić [44] have found "normal forms" for the elements of $P w B_{n}$, yet they do not decide whether $P w B_{n}$ is "automatic" in the sense of [37].

\subsection{Finite type invariants of v-braids and w-braids}

Just as we had two definitions for v-braids (and thus, for w-braids) in Section 2.1, we will give two equivalent developments of the theory of finite-type invariants of v-braids and w-braids: a pictorial/topological version in Section 2.3.1, and a more abstract algebraic version in Section 2.3.2. 


\subsubsection{Finite type invariants: the pictorial approach In the standard theory of finite-} type invariants of knots, also known as Vassiliev or Goussarov-Vassiliev invariants (see $[83 ; 43 ; 6 ; 13])$, one progresses from the definition of finite-type via iterated differences to chord diagrams and weight systems, to $4 T$ (and other) relations, to the definition of universal finite-type invariants, and beyond. The exact same progression (with different objects playing similar roles, and sometimes, when yet insufficiently studied, with the last step or two missing) is also seen in the theories of finite-type invariants of braids [9], 3-manifolds [77; 64; 61], virtual knots [42; 78] and of several other classes of objects. We thus assume that the reader has familiarity with these basic ideas, and we only indicate briefly how they are implemented in the case of v-braids and w-braids.

Much like the formula $X \rightarrow \nearrow-\lambda$ of Vassiliev-Goussarov fame, given a v-braid invariant $V: v B_{n} \rightarrow A$ valued in some abelian group $A$, we extend it to "singular" vbraids, ie braids that contain "semi-virtual crossings" like $\bar{x}$ and using the formulae $V\left({ }^{\prime}\right):=V(\%)-V(\times)$ and $V\left({ }^{\prime}\right):=V\left(\lambda^{7}\right)-V(\times)$ (see [42; 78; 20]). We say that " $V$ is of type $m$ " if its extension vanishes on singular v-braids having more than $m$ semi-virtual crossings. Up to invariants of lower type, an invariant of type $m$ is determined by its "weight system", which is a functional $W=W_{m}(V)$ defined on " $m$-singular v-braids modulo $\Upsilon=\chi=\lambda "$. Let us denote the vector space of all formal linear combinations of such equivalence classes by $\mathcal{G}_{m} \mathcal{D}_{n}^{v}$. Much as $m$-singular knots modulo $\nearrow=\lambda$ can be identified with chord diagrams, the basis elements of $\mathcal{G}_{m} \mathcal{D}_{n}^{v}$ can be identified with pairs $(D, \beta)$, where $D$ is a horizontal arrow diagram and $\beta$ is a "skeleton permutation", see Figure 2.

We assemble the spaces $\mathcal{G}_{m} \mathcal{D}_{n}^{v}$ together to form a single graded space,

$$
\mathcal{D}_{n}^{v}:=\bigoplus_{m=0}^{\infty} \mathcal{G}_{m} \mathcal{D}_{n}^{v} .
$$

Note that throughout this paper, whenever we write an infinite direct sum, we automatically complete it. Thus, in $\mathcal{D}_{n}^{v}$ we allow infinite sums with one term in each homogeneous piece $\mathcal{G}_{m} \mathcal{D}_{n}^{v}$; in particular, exponential-like sums will be heavily used.

In the standard finite-type theory for knots, weight systems always satisfy the $4 T$ relation, and are therefore functionals on $\mathcal{A}:=\mathcal{D} / 4 T$. Likewise, in the case of vbraids, weight systems satisfy the " $6 T$ relation" of $[42 ; 78 ; 20]$, shown in Figure 3 , and are therefore functionals on $\mathcal{A}_{n}^{v}:=\mathcal{D}_{n}^{v} / 6 T$. In the case of w-braids, the OC relation (9) implies the "tails commute" (TC) relation on the level of arrow diagrams, and in the presence of the TC relation, two of the terms in the $6 T$ relation drop out, and what remains is the " $\overrightarrow{4 \mathrm{~T}}$ " relation. These relations are shown in Figure 4. 

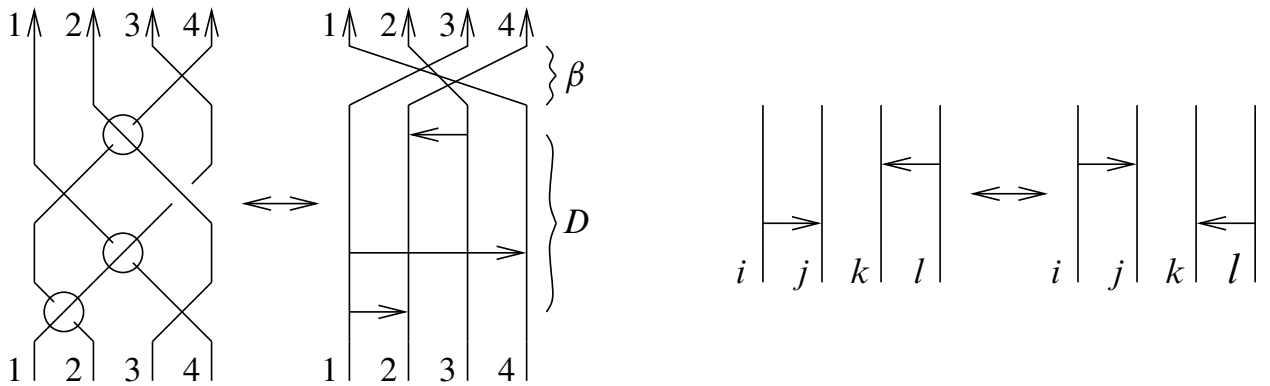

Figure 2: On the left, a 3-singular v-braid and its corresponding 3-arrow diagram. A self-explanatory algebraic notation for this arrow diagram is $\left(a_{12} a_{41} a_{23}, 3421\right)$. Note that we regard arrow diagrams as graph-theoretic objects, and hence, the two arrow diagrams on the right, whose underlying graphs are the same, are regarded as equal. In algebraic notation this means that we always impose the relation $a_{i j} a_{k l}=a_{k l} a_{i j}$ when the indices $i, j$, $k$, and $l$ are all distinct.

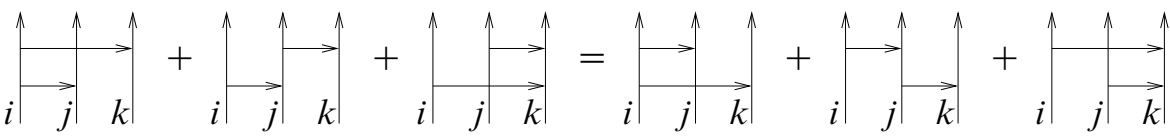

$$
\begin{gathered}
a_{i j} a_{i k}+a_{i j} a_{j k}+a_{i k} a_{j k}=a_{i k} a_{i j}+a_{j k} a_{i j}+a_{j k} a_{i k} \\
\text { or } \quad\left[a_{i j}, a_{i k}\right]+\left[a_{i j}, a_{j k}\right]+\left[a_{i k}, a_{j k}\right]=0 .
\end{gathered}
$$

Figure 3: The $6 T$ relation. Standard knot theoretic conventions apply: only the relevant parts of each diagram are shown; in reality each diagram may have further vertical strands and horizontal arrows, provided the extras are the same in all 6 diagrams. Also, the vertical strands are in no particular order other valid $6 T$ relations are obtained when those strands are permuted in other ways.

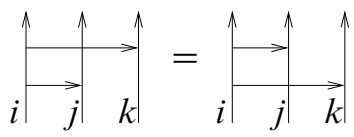

$a_{i j} a_{i k}=a_{i k} a_{i j}$

or $\left[a_{i j}, a_{i k}\right]=0$.

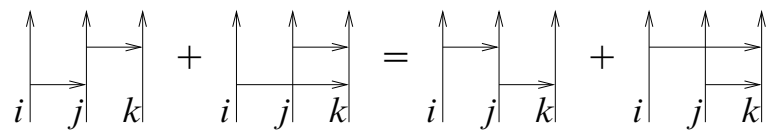

$a_{i j} a_{j k}+a_{i k} a_{j k}=a_{j k} a_{i j}+a_{j k} a_{i k}$

or $\left[a_{i j}+a_{i k}, a_{j k}\right]=0$.

Figure 4: The TC and the $\overrightarrow{4 \mathrm{~T}}$ relations.

Thus, weight systems of finite-type invariants of w-braids are linear functionals on $\mathcal{A}_{n}^{w}:=\mathcal{D}_{n}^{v} / \mathrm{TC}, \overrightarrow{4 \mathrm{~T}}$. 
The next question that arises is whether we have already found all the relations that weight systems always satisfy. More precisely, given a degree $m$ linear functional on $\mathcal{A}_{n}^{v}=\mathcal{D}_{n}^{v} / 6 T$ (or $\mathcal{A}_{n}^{w}=\mathcal{D}_{n}^{v} / \mathrm{TC}, \overrightarrow{4 \mathrm{~T}}$ ), is it always the weight system of some type $m$ invariant $V$ of v-braids (or w-braids)? As in every other theory of finite-type invariants, the answer to this question is affirmative if and only if there exists a "universal finite-type invariant" (or simply, an "expansion") of v-braids (or w-braids), defined as follows.

Definition 2.11 An expansion for v-braids (or w-braids) is an invariant $Z: v B_{n} \rightarrow \mathcal{A}_{n}^{v}$ (or $Z: w B_{n} \rightarrow \mathcal{A}_{n}^{w}$ ) satisfying the following "universality condition":

- If $B$ is an $m$-singular v-braid (or w-braid) and $D \in \mathcal{G}_{m} \mathcal{D}_{n}^{v}$ is its underlying arrow diagram as in Figure 2, then

$$
Z(B)=D+(\text { terms of degree }>m) .
$$

Indeed if $Z$ is an expansion and $W \in \mathcal{G}_{m} \mathcal{A}^{\star},{ }^{15}$ the universality condition implies that $W \circ Z$ is a finite-type invariant whose weight system is $W$. To go the other way, if $\left(D_{i}\right)$ is a basis of $\mathcal{A}$ consisting of homogeneous elements, if $\left(W_{i}\right)$ is the dual basis of $\mathcal{A}^{\star}$ and $\left(V_{i}\right)$ are finite-type invariants whose weight systems are the $W_{i}$, then $Z(B):=\sum_{i} D_{i} V_{i}(B)$ defines an expansion.

In general, constructing a universal finite-type invariant is a hard task. For knots, one uses either the Kontsevich integral or perturbative Chern-Simons theory (also known as "configuration space integrals" [27] or "tinker-toy towers" [82]) or the rather fancy algebraic theory of "Drinfel'd associators" (a summary of all those approaches is in [23]). For homology spheres, this is the "LMO invariant" [64; 61] (also the "Århus integral" [17; 18; 19]). For v-braids, an expansion exists by a difficult result of P. Lee [66]. In contrast, as we shall see below, the construction of an expansion for w-braids is quite easy.

2.3.2 Finite type invariants: the algebraic approach For any group $G$, one can form the group algebra $\mathbb{Q} G$ by allowing formal linear combinations of group elements and extending multiplication linearly, where $\mathbb{Q}$ is the field of rational numbers. ${ }^{16}$ The augmentation ideal is the ideal generated by differences, or equivalently, the set of linear combinations of group elements whose coefficients sum to zero:

$$
\mathcal{I}:=\left\{\sum_{i=1}^{k} a_{i} g_{i}: a_{i} \in \mathbb{Q}, g_{i} \in G, \sum_{i=1}^{k} a_{i}=0\right\} .
$$

${ }^{15} \mathcal{A}^{\star}$ here denotes either $\mathcal{A}_{n}^{v}$ or $\mathcal{A}_{n}^{w}$, or in fact any of many similar spaces that we will discuss later.

${ }^{16}$ The definitions in this subsection make sense over $\mathbb{Z}$ as well, but the main result of the next subsection requires a field of characteristic 0 . For simplicity of notation we stick with $\mathbb{Q}$. 
Powers of the augmentation ideal provide a filtration of the group algebra. We denote by

$$
\mathcal{A}(G):=\bigoplus_{m \geq 0} \mathcal{I}^{m} / \mathcal{I}^{m+1}
$$

the associated graded space corresponding to this filtration.

Definition 2.12 An expansion for the group $G$ is a map $Z: G \rightarrow \mathcal{A}(G)$ such that the linear extension $Z: \mathbb{Q} G \rightarrow \mathcal{A}(G)$ is filtration-preserving and the induced map

$$
\text { gr } Z:(\operatorname{gr} \mathbb{Q} G=\mathcal{A}(G)) \rightarrow(\operatorname{gr} \mathcal{A}(G)=\mathcal{A}(G))
$$

is the identity. An equivalent way to phrase this is that the degree $m$ piece of $Z$ restricted to $\mathcal{I}^{m}$ is the projection onto $\mathcal{I}^{m} / \mathcal{I}^{m+1}$.

Exercise 2.13 Verify that for the groups $P v B_{n}$ and $P w B_{n}$ the $m^{\text {th }}$ power of the augmentation ideal coincides with the span of all resolutions of $m$-singular v- or wbraids (by a resolution we mean the formal linear combination where each semivirtual crossing is replaced by the appropriate difference of a virtual and a regular crossing, as in Figure 2). Then check that the notion of expansion defined above is the same as that of Definition 2.11, restricted to pure braids.

Finally, note the functorial nature of the construction above. What we have described is a functor gr from the category of groups to the category of graded algebras, which assigns to each group $G$ the graded algebra $\mathcal{A}(G)$. To each homomorphism $\phi: G \rightarrow H$, gr assigns the induced map

$$
\operatorname{gr} \phi:(\mathcal{A}(G)=\operatorname{gr} \mathbb{Q} G) \rightarrow(\mathcal{A}(H)=\operatorname{gr} \mathbb{Q} H) .
$$

\subsection{Expansions for w-braids}

The space $\mathcal{A}_{n}^{w}$ of arrow diagrams on $n$ strands is an associative algebra in an obvious manner: if the permutations underlying two arrow diagrams are the identity permutations, then we simply juxtapose the diagrams. Otherwise we "slide" arrows through permutations in the obvious manner: if $\tau$ is a permutation, we declare that $\tau a_{(\tau i)(\tau j)}=a_{i j} \tau$. Instead of seeking an expansion $w B_{n} \rightarrow \mathcal{A}_{n}^{w}$, we set the bar a little higher and seek a "homomorphic expansion", defined as follows.

Definition 2.14 A homomorphic expansion $Z: w B_{n} \rightarrow \mathcal{A}_{n}^{w}$ is an expansion that carries products in $w B_{n}$ to products in $\mathcal{A}_{n}^{w}$. 
To find a homomorphic expansion, we just need to define it on the generators of $w B_{n}$ and verify that it satisfies the relations defining $w B_{n}$ and the universality condition. Following [26, Section 5.3] and [2, Section 8.1] we set $Z(X)=X$ (that is, a transposition in $w B_{n}$ gets mapped to the same transposition in $\mathcal{A}_{n}^{w}$, adding no arrows) and $Z(夭)=\exp (\hat{\wedge} \uparrow) \times$. (Recall that we work in the degree completion.) This last formula is important so deserves to be magnified, explained and replaced by some new notation:

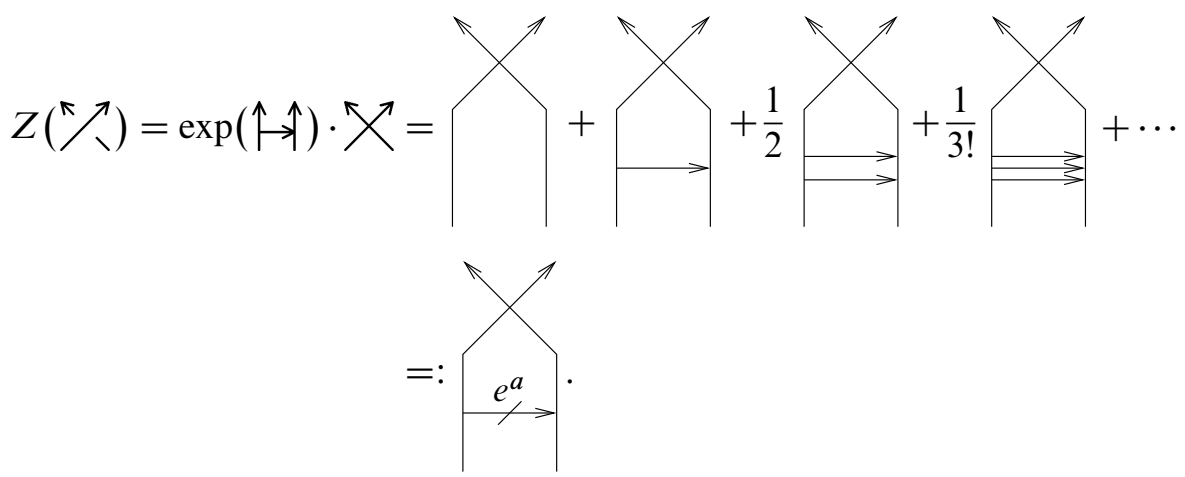

Thus the new notation $\stackrel{e^{a}}{\longrightarrow}$ stands for an "exponential reservoir" of parallel arrows, much like

$$
e^{a}=1+a+a a / 2+a a a / 3 !+\cdots
$$

is a "reservoir" of $a$ 's. With the obvious interpretation for $\stackrel{e^{-a}}{\longrightarrow}$ (that is, the - sign indicates that the terms should have alternating signs, as in $e^{-a}=1-a+a^{2} / 2-$ $a^{3} / 3$ ! $\left.+\cdots\right)$, the second Reidemeister move $>\lambda^{n}=1$ forces that we set

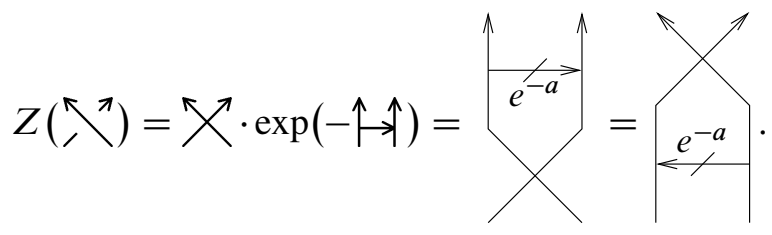

Theorem 2.15 The above formulae define an invariant $Z: w B_{n} \rightarrow \mathcal{A}_{n}^{w}$ (that is, $Z$ satisfies all the defining relations of $w B_{n}$ ). The resulting $Z$ is a homomorphic expansion (that is, it satisfies the universality property of Definition 2.14).

Proof Following [26; 2]: for the invariance of $Z$, the only interesting relations to check are the Reidemeister 3 relation of (4) and the OC relation of (10). For Reidemeister 3, 
we have

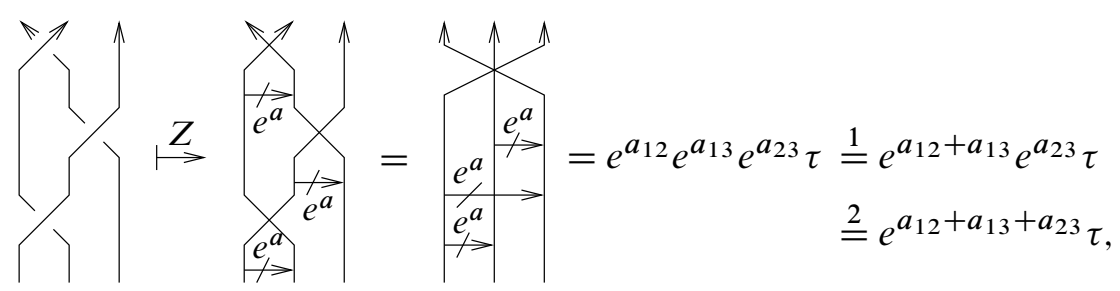

where $\tau$ is the permutation 321 , equality 1 holds because a TC relation implies $\left[a_{12}, a_{13}\right]=0$, and equality 2 holds because $\left[a_{12}+a_{13}, a_{23}\right]=0$ by a $\overrightarrow{4 T}$ relation. Likewise, again using TC and $\overrightarrow{4 T}$,

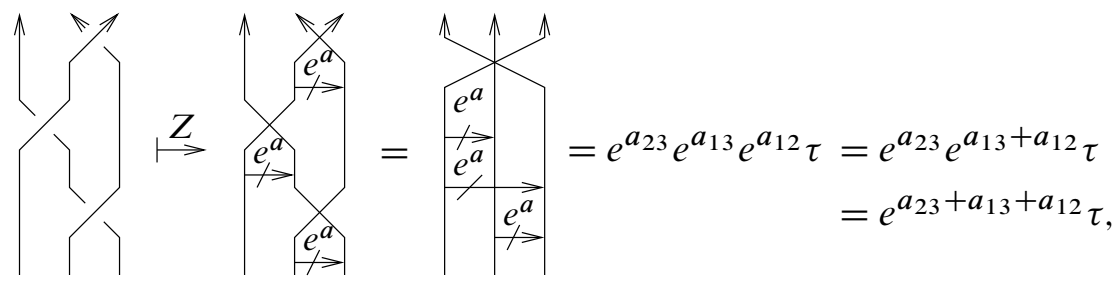

and so Reidemeister 3 holds. An even simpler proof using just the TC relation shows that the OC relation also holds. Finally, since $Z$ is homomorphic, it is enough to check the universality property at degree 1 , where it is very easy:

$$
Z(\varnothing)=\exp (\uparrow \uparrow) \cdot X-\nwarrow=\uparrow \uparrow \cdot X+(\text { terms of degree }>1)
$$

A similar computation manages the $\$$ case.

Remark 2.16 Note that the main ingredient of the above proof was to show that $R:=Z\left(\sigma_{12}\right)=e^{a_{12}}$ satisfies the famed Yang-Baxter equation,

$$
R^{12} R^{13} R^{23}=R^{23} R^{13} R^{12}
$$

where $R^{i j}$ means "place $R$ on strands $i$ and $j$ ".

\subsection{Some further comments}

2.5.1 Compatibility with braid operations As with any new gadget, we would like to know how compatible the expansion $Z$ of the previous section is with the gadgets we already have; namely, with various operations that are available on w-braids and with the action of w-braids on the free group $F_{n}$; see Section 2.2.3. 
2.5.1.1 $Z$ is compatible with braid inversion Let $\theta$ denote both the "braid inversion" operation $\theta: w B_{n} \rightarrow w B_{n}$ defined by $B \mapsto B^{-1}$ and the "antipode" antiautomorphism $\theta: \mathcal{A}_{n}^{w} \rightarrow \mathcal{A}_{n}^{w}$ defined by mapping permutations to their inverses and arrows to their negatives (that is, $a_{i j} \mapsto-a_{i j}$ ). Then the diagram below commutes:

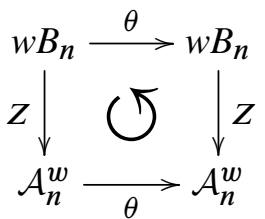

2.5.1.2 Braid cloning and the group-like property Let $\Delta$ denote both the "braid cloning" operation $\Delta: w B_{n} \rightarrow w B_{n} \times w B_{n}$ defined by $B \mapsto(B, B)$ and the "co-product" algebra morphism $\Delta: \mathcal{A}_{n}^{w} \rightarrow \mathcal{A}_{n}^{w} \otimes \mathcal{A}_{n}^{w}$ defined by cloning permutations (that is, $\tau \mapsto \tau \otimes \tau$ ) and by treating arrows as primitives (that is, $a_{i j} \mapsto a_{i j} \otimes 1+1 \otimes a_{i j}$ ). Then the diagram below commutes:

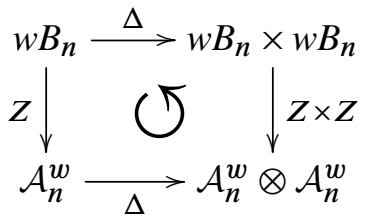

In formulae, this is $\Delta(Z(B))=Z(B) \otimes Z(B)$, which is the statement " $Z(B)$ is group-like".

2.5.1.3 Strand insertions Let $\iota: w B_{n} \rightarrow w B_{n+1}$ be an operation of "inert strand insertion". Given $B \in w B_{n}$, the resulting $\iota B \in w B_{n+1}$ will be $B$ with one strand $S$ added at some location chosen in advance: to the left of all existing strands, or to the right, or starting from between the $3^{\text {rd }}$ and the $4^{\text {th }}$ strand of $B$ and ending between the $6^{\text {th }}$ and the $7^{\text {th }}$ strand of $B$; when adding $S$, add it "inert", so that all crossings on it are virtual (this is well-defined). There is a corresponding inert strand addition operation $\iota: \mathcal{A}_{n}^{w} \rightarrow \mathcal{A}_{n+1}^{w}$, obtained by adding a strand at the same location as for the original $\iota$ and adding no arrows. It is easy to check that $Z$ is compatible with $\iota$; namely, that the following diagram is commutative:

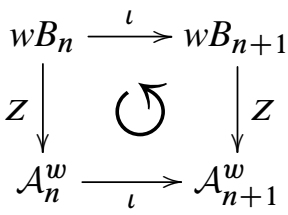


2.5.1.4 Strand deletions Given $1 \leq k \leq n$, let $d_{k}: w B_{n} \rightarrow w B_{n-1}$ be the operation of "removing the $k^{\text {th }}$ strand". This operation induces a homonymous operation $d_{k}: \mathcal{A}_{n}^{w} \rightarrow$ $\mathcal{A}_{n-1}^{w}$ : if $D \in \mathcal{A}_{n}^{w}$ is an arrow diagram, then $d_{k} D$ is $D$ with its $k^{\text {th }}$ strand removed if no arrows in $D$ start or end on the $k^{\text {th }}$ strand, and it is 0 otherwise. It is easy to check that $Z$ is compatible with $d_{k}$; namely, that the diagram below is commutative: ${ }^{17}$

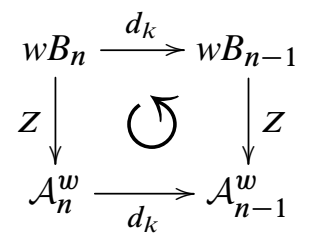

2.5.1.5 Compatibility with the action on $\boldsymbol{F}_{\boldsymbol{n}}$ Let $F A_{n}$ denote the (degree-completed) free, associative (but not commutative) algebra on the generators $x_{1}, \ldots, x_{n}$. Then there is an "expansion" $Z: F_{n} \rightarrow F A_{n}$ defined by $\xi_{i} \mapsto e^{x_{i}}$ (see [72] and the related "Magnus Expansion" of [73]). Also, there is a right action ${ }^{18}$ of $\mathcal{A}_{n}^{w}$ on $F A_{n}$ defined on generators by $x_{i} \tau=x_{\tau i}$ for $\tau \in S_{n}$ and by $x_{j} a_{i j}=\left[x_{i}, x_{j}\right]$ and $x_{k} a_{i j}=0$ for $k \neq j$ and extended by the Leibniz rule to the rest of $F A_{n}$ and then multiplicatively to the rest of $\mathcal{A}_{n}^{w}$. This fits into the diagram below:

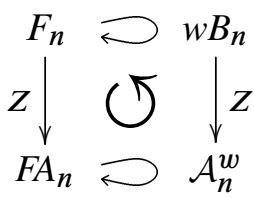

Exercise 2.17 Use the definition of the action in (14) and the commutative diagrams of paragraphs 2.5.1.1, 2.5.1.2 and 2.5.1.3 to show that the diagram of paragraph 2.5.1.5 is also commutative.

${ }^{17}$ In [16] we'll say that " $d_{k}: w B_{n} \rightarrow w B_{n-1}$ " is an algebraic structure made of two spaces ( $w B_{n}$ and $w B_{n-1}$ ), two binary operations (braid composition in $w B_{n}$ and in $w B_{n-1}$ ), and one unary operation, $d_{k}$. After applying gr we get the algebraic structure $d_{k}: \mathcal{A}_{n}^{w} \rightarrow \mathcal{A}_{n-1}^{w}$ with $d_{k}$ as described above, and an alternative way of stating our assertion is to say that $Z$ is a morphism of algebraic structures. A similar remark applies (sometimes in the negative form) to the other operations discussed in this section.

${ }^{18}$ In the language of [16], we will say that $F A_{n}=\mathrm{gr} F_{n}$ and that when the actions involved are regarded as instances of the algebraic structure "one monoid acting on another", we have that $\left(F A_{n} \triangleright \mathcal{A}_{n}^{w}\right)=$ $\operatorname{gr}\left(F_{n} \supset w B_{n}\right)$. 
2.5.1.6 Unzipping a strand Given $k$ between 1 and $n$, let $u_{k}: w B_{n} \rightarrow w B_{n+1}$ be the operation of "unzipping the $k^{\text {th }}$ strand", briefly defined as follows: ${ }^{19}$

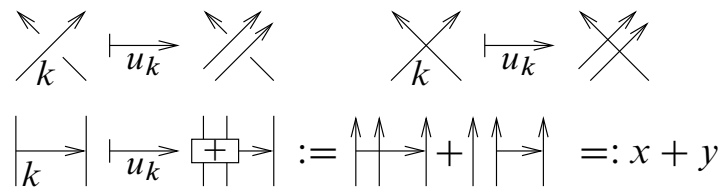

The induced operation $u_{k}: \mathcal{A}_{n}^{w} \rightarrow \mathcal{A}_{n+1}^{w}$ is also shown: if an arrow starts (or ends) on the strand being doubled, it is replaced by a sum of two arrows that start (or end) on either of the two "daughter strands". This is performed for each arrow independently; so if there are $t$ arrows touching the $k^{\text {th }}$ strands in a diagram $D$, then $u_{k} D$ will be a sum of $2^{t}$ diagrams.

In some sense, much of this current series of papers as well as the works of Kashiwara and Vergne [52] and Alekseev and Torossian [2] are about coming to grips with the fact that $Z$ is not compatible with $u_{k}$; that is, the diagram

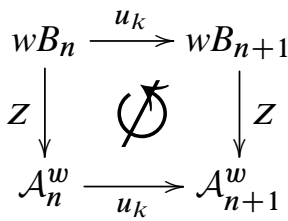

is not commutative. Indeed, let $x:=a_{13}$ and $y:=a_{23}$ be as above, and let $s$ be the permutation 21 and $\tau$ the permutation 231. Then $d_{1} Z\left({ }^{\top} /\right)=d_{1}\left(e^{a_{12}} s\right)=e^{x+y} \tau$, while $Z\left(d_{1}{ }^{x}\right)=e^{y} e^{x} \tau$. So the failure of $d_{1}$ and $Z$ to commute is the ill behaviour of the exponential function when its arguments do not commute, which is measured by the $\mathrm{BCH}$ formula, central to both [52] and [2].

2.5.2 Power and injectivity The following theorem is due to Berceanu and $\mathrm{Pa}-$ padima [26, Theorem 5.4]; a variant of this theorem is also true for ordinary braids [57; $7 ; 45]$, and can be proven by similar means.

Theorem 2.18 $Z: w B_{n} \rightarrow \mathcal{A}_{n}^{w}$ is injective. In other words, finite-type invariants separate $w$-braids.

Proof The statement follows immediately from the faithfulness of the action $F_{n} \supset w B_{n}$, from the compatibility of $Z$ with this action, and from the injectivity of $Z: F_{n} \rightarrow F A_{n}$

${ }^{19}$ Unzipping a knotted zipper turns a single band into two parallel ones. This operation is also known as "strand doubling", but for compatibility with operations that will be introduced later, we prefer "unzipping". 
(the latter is well known, see eg [73, Theorem 5.6] ${ }^{20}$ and [72]). Indeed, if $B_{1}$ and $B_{2}$ are w-braids and $Z\left(B_{1}\right)=Z\left(B_{2}\right)$, then $Z(\xi) Z\left(B_{1}\right)=Z(\xi) Z\left(B_{2}\right)$ for any $\xi \in F_{n}$. Therefore $\forall \xi, Z\left(\xi / / B_{1}\right)=Z\left(\xi / / B_{2}\right)$, therefore $\forall \xi, \xi / / B_{1}=\xi / / B_{2}$, therefore $B_{1}=B_{2}$.

Remark 2.19 Apart from the easy fact that $\mathcal{A}_{n}^{w}$ can be computed degree by degree in exponential time, we do not know a simple formula for the dimension of the degree $m$ piece of $\mathcal{A}_{n}^{w}$ or a natural basis of that space. This compares unfavourably with the situation for ordinary braids (see eg [9]). Also compare with Problem 2.9 and with Remark 2.10.

2.5.3 Uniqueness There is certainly not a unique expansion for w-braids: if $Z_{1}$ is an expansion and $P$ is any degree-increasing linear map $\mathcal{A}^{w} \rightarrow \mathcal{A}^{w}$ (a "pollution" map), then $Z_{2}:=(I+P) \circ Z_{1}$ is also an expansion, where $I: \mathcal{A}^{w} \rightarrow \mathcal{A}^{w}$ is the identity. But that's all, and if we require a bit more, even that freedom disappears.

Proposition 2.20 If $Z_{1,2}: w B_{n} \rightarrow \mathcal{A}_{n}^{w}$ are expansions then there exists a degreeincreasing linear map $P: \mathcal{A}^{w} \rightarrow \mathcal{A}^{w}$ such that $Z_{2}=(I+P) \circ Z_{1}$.

Sketch of proof Let $\widehat{w B_{n}}$ be the unipotent completion of $w B_{n}$. That is, let $\mathbb{Q} w B_{n}$ be the algebra of formal linear combinations of w-braids, let $\mathcal{I}$ be the ideal in $\mathbb{Q} w B_{n}$ generated by $\bar{x}=\aleph-X$ and by $\bar{X}=\bar{X}-\lambda$, and set

$$
\widehat{w B_{n}}:=\lim _{m \rightarrow \infty} \mathbb{Q} w B_{n} / \mathcal{I}^{m}
$$

Here $\widehat{w B_{n}}$ is filtered with

$$
\mathcal{F}_{m} \widehat{w B_{n}}:=\lim _{m^{\prime}>m} \mathcal{I}^{m} / \mathcal{I}^{m^{\prime}}
$$

An "expansion" can be re-interpreted as an "isomorphism of $\widehat{w B_{n}}$ and $\mathcal{A}_{n}^{w}$ as filtered vector spaces". Always, any two isomorphisms differ by an automorphism of the target space, and that's the essence of $I+P$.

Proposition 2.21 If $Z_{1,2}: w B_{n} \rightarrow \mathcal{A}_{n}^{w}$ are homomorphic expansions that commute with braid cloning (Section 2.5.1.2) and with strand insertion (Section 2.5.1.3), then $Z_{1}=Z_{2}$.

${ }^{20}$ Though notice that we use $\xi_{i} \mapsto e^{x_{i}}$ whereas [73, Theorem 5.6] uses $\xi_{i} \mapsto 1+x_{i}$. The injectivity proof of [73] holds in our case just as well. 
Sketch of proof A homomorphic expansion that commutes with strand insertions is determined by its values on the generators $X, \lambda$ and $X$ of $w B_{2}$. Commutativity with braid cloning (see Section 2.5.1.2) implies that these values must be, up to permuting the strands, group-like: that is, the exponentials of primitives. But the only primitives are $a_{12}$ and $a_{21}$, and one may easily verify that there is only one way to arrange these so that $Z$ will respect $X^{2}=X \cdot \lambda^{n}=1$ and $\boldsymbol{x}^{2} \mapsto \hat{\mapsto} \hat{x}+$ (higher degree terms).

2.5.4 The group of non-horizontal flying rings Let $Y_{n}$ denote the space of all placements of $n$ numbered disjoint oriented unlinked geometric circles in $\mathbb{R}^{3}$. Such a placement is determined by the centres in $\mathbb{R}^{3}$ of the circles, the radii, and a unit normal vector for each circle pointing in the positive direction, so $\operatorname{dim} Y_{n}=3 n+n+3 n=7 n$. $S_{n} \ltimes \mathbb{Z}_{2}^{n}$ acts on $Y_{n}$ by permuting the circles and mapping each circle to its image in either an orientation-preserving or an orientation-reversing way. Let $\tilde{Y}_{n}$ denote the quotient $Y_{n} / S_{n} \ltimes \mathbb{Z}_{2}^{n}$. The fundamental group $\pi_{1}\left(\tilde{Y}_{n}\right)$ can be thought of as the "group of flippable flying rings". Without loss of generality, we can assume that the basepoint is chosen to be a horizontal placement. We want to study the relationship of this group to $w B_{n}$.

Theorem 2.22 The group $\pi_{1}\left(\tilde{Y}_{n}\right)$ is a $\mathbb{Z}_{2}^{n}$-extension of $w B_{n}$, generated by $s_{i}, \sigma_{i}$ $(1 \leq i \leq n-1)$, and $w_{i}$ ("flips") for $1 \leq i \leq n$, with the relations as above, and in addition:

$$
\begin{aligned}
& w_{i}^{2}=1 ; \quad w_{i} w_{j}=w_{j} w_{i} ; \quad w_{j} s_{i}=s_{i} w_{j} \quad \text { when } i \neq j, j+1 ; \\
& w_{i} s_{i}=s_{i} w_{i+1} ; \quad w_{i+1} s_{i}=s_{i} w_{i} ; \quad w_{j} \sigma_{i}=\sigma_{i} w_{j} \quad \text { if } j \neq i, i+1 \text {; } \\
& w_{i+1} \sigma_{i}=\sigma_{i} w_{i} ; \quad \text { yet } \quad w_{i} \sigma_{i}=s_{i} \sigma_{i}^{-1} s_{i} w_{i+1} \text {. }
\end{aligned}
$$

The two most interesting flip relations in pictures:

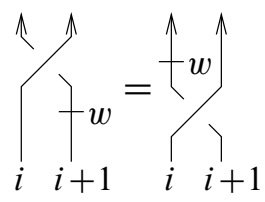

yet

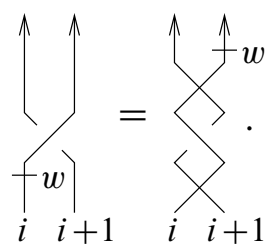

Instead of a proof, we provide some heuristics. Since each circle starts out in a horizontal position and returns to a horizontal position, there is an integer number of "flips" they 
do in between; these are the generators $w_{i}$, as shown below:

$$
w_{i}=w-\rightarrow \begin{gathered}
0 \\
0 \\
0 \\
0 \\
0
\end{gathered}
$$

The first relation says that a double flip is homotopic to doing nothing. Technically, there are two different directions of flips, and they are the same via this (non-obvious but true) relation. The rest of the first line is obvious: flips of different rings commute, and if two rings fly around each other while another one flips, the order of these events can be switched by homotopy. The second line says that if two rings trade places with no interaction while one flips, then the order of these events can be switched as well. However, we have to re-number the flip to conform to the strand labelling convention.

The only subtle point is how flips interact with crossings. First of all, if one ring flies through another while a third one flips, the order clearly does not matter. If a ring flies through another and also flips, the order can be switched. However, if ring $A$ flips and then ring $B$ flies through it, this is homotopic to $\operatorname{ring} B$ flying through $\operatorname{ring} A$ from the other direction and then ring $A$ flipping. In other words, commuting $\sigma_{i}$ with $w_{i}$ changes the "sign of the crossing" in the sense of Exercise 2.7. This gives the last, and the only truly non-commutative flip relation.

To explain why the flip is denoted by $w$, let us consider the alternative description by ribbon tubes. A flipping ring traces a so-called wen ${ }^{21}$ in $\mathbb{R}^{4}$. A wen is a Klein bottle cut along a meridian circle, as shown below. The wen is embedded in $\mathbb{R}^{4}$.

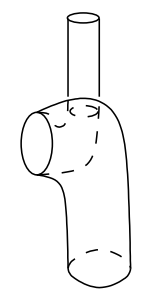

Finally, note that $\pi_{1} Y_{n}$ is exactly the pure w-braid group $P w B_{n}$ : since each ring has to return to its original position and orientation, each does an even number of flips. The flips (or wens) can all be moved to the bottoms of the braid diagram strands (to the bottoms of the tubes, to the beginning of words), at a possible cost, as specified by Equation (16). Once together, they pairwise cancel each other. As a result, this group can be thought of as not containing wens at all.

${ }^{21}$ The term wen was coined by Kanenobu and Shima in [51]. 
2.5.5 The relationship with u-braids For the sake of ignoring strand permutations, we restrict our attention to pure braids.

By Section 2.3.2, for any expansion $Z^{u}: P u B_{n} \rightarrow \mathcal{A}_{n}^{u}$, where $P u B_{n}$ is the "usual" braid group and $\mathcal{A}_{n}^{u}$ is the algebra of horizontal chord diagrams on $n$ strands, there is a square of maps as follows:

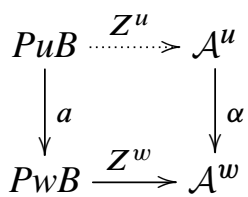

Here $Z^{w}$ is the expansion constructed in Section 2.4, the left vertical map $a$ is the composition of the inclusion and projection maps $P u B_{n} \rightarrow P v B_{n} \rightarrow P w B_{n}$. The map $\alpha$ is the induced map by the functoriality of gr, as noted after Exercise 2.13. The reader can verify that $\alpha$ maps each chord to the sum of its two possible directed versions.

Note that this square is not commutative for any choice of $Z^{u}$ even in degree 2: the image of a crossing under $Z^{w}$ is outside the image of $\alpha$.

More specifically, for any choice $c$ of a "parenthesization" of $n$ points, the KZconstruction/Kontsevich integral (see for example [10]) returns an expansion $Z_{c}^{u}$ of u-braids:

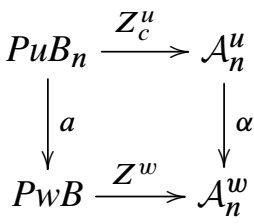

We shall see in [16, Proposition 4.15] that for any choice of $c$, the two compositions $\alpha \circ Z_{c}^{u}$ and $Z^{w} \circ a$ are "conjugate in a bigger space": there is a map $i$ from $\mathcal{A}^{w}$ to a larger space of "non-horizontal arrow diagrams", and in this space the images of the above composites are conjugate. However, we are not certain that $i$ is an injection, and whether the conjugation leaves the $i$-image of $\mathcal{A}^{w}$ invariant, and so we do not know if the two compositions differ merely by an outer automorphism of $\mathcal{A}^{w}$.

\section{3 w-knots}

In Section 3.1 we define v-knots and w-knots (long v-knots and long w-knots, to be precise) and discuss a map $v \rightarrow w$. In Section 3.2 we determine the space of "chord diagrams" for w-knots to be the space $\mathcal{A}^{w}(\uparrow)$ of arrow diagrams modulo $\overrightarrow{4 T}$ and TC relations, and in Section 4.1 we compute some relevant dimensions. In Section 3.4 we 
show that $\mathcal{A}^{w}(\uparrow)$ can be re-interpreted as a space of trivalent graphs modulo STU- and IHX-like relations, and is therefore related to Lie algebras (Section 3.5). This allows us to completely determine $\mathcal{A}^{w}(\uparrow)$. With no difficulty in Section 3.3 we construct a universal finite-type invariant for w-knots. With a bit of further difficulty we show in Section 3.6 that it is essentially equal to the Alexander polynomial.

Knots are the wrong objects for study in knot theory, v-knots are the wrong objects for study in the theory of v-knotted objects and w-knots are the wrong objects for study in the theory of w-knotted objects. Studying uvw-knots on their own is the parallel of studying cakes, cookies and pastries as they come out of the bakery: we sure want to make them our own, but the theory of desserts is more about the ingredients and how they are put together than about the end products. In algebraic knot theory this reflects through the fact that knots are not finitely generated in any sense (hence, they must be made of some more basic ingredients), and through the fact that there are very few operations defined on knots (connected sums and satellite operations being the main exceptions), and thus, most interesting properties of knots are transcendental, or non-algebraic, when viewed from within the algebra of knots and operations on knots [12].

The right objects for study in knot theory, or v-knot theory or w-knot theory, are thus the ingredients that make up knots and that permit a richer algebraic structure. These are braids, studied in the previous section, and even more so tangles and tangled graphs, studied in [16]. Yet tradition has its place and the sweets are tempting, and we can introduce and apply some of the tools we will use in the deeper and healthier study of $\mathrm{w}$-tangles and w-tangled foams in the limited, but tasty, arena of the baked goods of knot theory, the knots themselves.

\section{1 v-knots and w-knots}

v-knots may be understood either as knots drawn on surfaces modulo the addition or removal of empty handles [59], or as "Gauss diagrams" (see Remark 3.4), or simply "unembedded but wired together" crossings modulo the Reidemeister moves (see [54; 80] and [16, Section 2]). But right now we forgo the topological and the abstract and give only the "planar" (and somewhat less philosophically satisfying) definition of v-knots.

Definition 3.1 A "long v-knot diagram" is an arc smoothly drawn in the plane from $-\infty$ to $+\infty$, with finitely many self-intersections, divided into "virtual crossings" $X$, overcrossings $~ \nearrow$ (aka positive crossings), and undercrossings $\lambda$ (aka negative crossings); and regarded up to planar isotopy. 


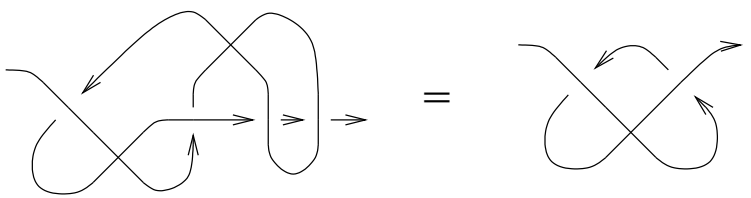

Figure 5: A long v-knot diagram with 2 virtual crossings, 2 positive crossings and 2 negative crossings. A positive-negative pair can easily be cancelled using R2, and then a virtual crossing can be cancelled using VR1, and it seems that the rest cannot be simplified any further.

A picture is worth more than a more formal definition, and one appears in Figure 5. A "long v-knot" is an equivalence class of long v-knot diagrams, modulo the equivalence generated by the Reidemeister $1^{s}, 2$ and 3 moves (R1 ${ }^{s}, \mathrm{R} 2$ and R3), ${ }^{22}$ the virtual Reidemeister 1 through 3 moves (VR1, VR2, VR3), and by the mixed relations (M); all these are shown in Figure 6. Finally, "long w-knots" are obtained from long v-knots by also dividing by the overcrossings commute (OC) relations, also shown in Figure 6. Note that we never mod out by the Reidemeister 1 (R1) move nor by the undercrossings commute relation (UC).

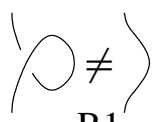

R1

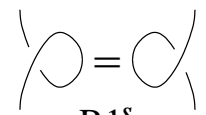

$\mathrm{R} 1^{s}$

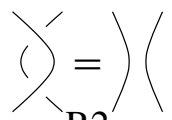

$\mathrm{R} 2$

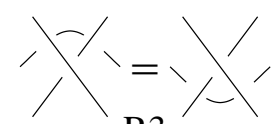

R3

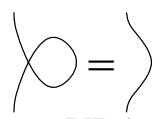

VR1

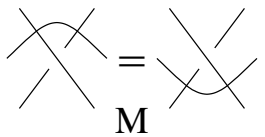

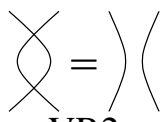

VR2

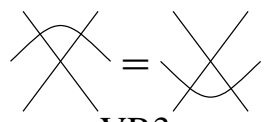

VR3
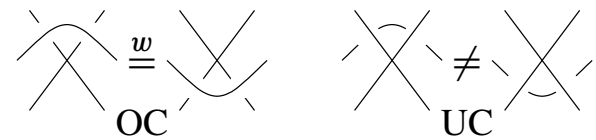

Figure 6: The relations defining v-knots and w-knots, along with two relations that are not imposed.

Definition and warning 3.2 A "circular v-knot" is like a long v-knot, except that it is parametrized by a circle rather than by a long line. Unlike the case of usual knots, circular v-knots are not equivalent to long v-knots [54]. The same applies to w-knots.

${ }^{22} \mathrm{R} 1$ ' is the "spun" version of R1: kinks can be spun around, but not removed outright. See Figure 6. 
Definition and warning 3.3 Long v-knots form a monoid using the concatenation operation \#. Unlike the case of usual knots, the resulting monoid is not abelian [54]. The same applies to w-knots.

Remark 3.4 A "Gauss diagram" is a straight "skeleton line" along with signed directed chords (signed "arrows") marked along it (more in [54; 42]). Gauss diagrams are in obvious bijection with long v-knot diagrams; the skeleton line of a Gauss diagram corresponds to the parameter space of the v-knot, and the arrows correspond to the crossings, with each arrow heading from the upper strand to the lower strand, marked by the sign of the relevant crossing:
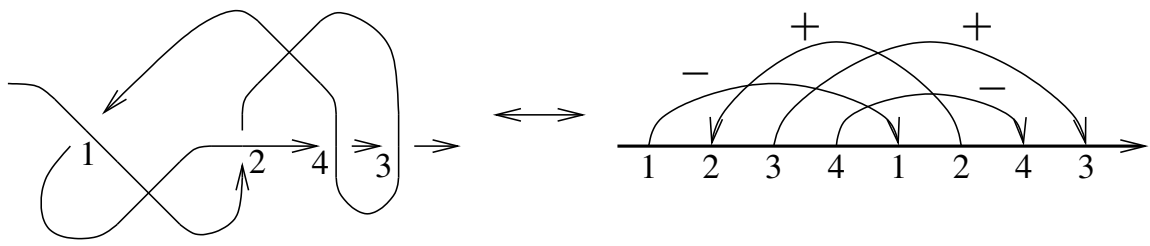

One may also describe the relations in Figure 6 as well as circular v-knots and other types of v-knots (as we will encounter later) in terms of Gauss diagrams with varying skeletons.

Remark 3.5 Since we do not mod out by R1, it is perhaps more appropriate to call our class of v/w-knots "framed long v/w-knots", but since we care more about framed $\mathrm{v} / \mathrm{w}$-knots than about unframed ones, we reserve the unqualified name for the framed case, and when we do wish to mod out by R1 we will explicitly write "unframed long v/w-knots".

Recall that in the case of "usual knots", or u-knots, dropping the R1 relation altogether also results in a $\mathbb{Z}^{2}$-extension of unframed knot theory, where the two factors of $\mathbb{Z}$ are framing and rotation number. If one wants to talk about "true" framed knots, one mods out by the spun Reidemeister 1 relation ( $\mathrm{R} 1^{s}$ of Figure 6), which preserves the blackboard framing but does not preserve the rotation number. We take the analogous approach here, including the $\mathrm{R} 1^{s}$ relation — but not $\mathrm{R} 1$ - in the $\mathrm{v}$ and $\mathrm{w}$ cases.

This said, note that the monoid of long v-knots is just a central extension by $\mathbb{Z}$ of the monoid of unframed long $v$-knots, and so studying the framed case is not very different from studying the unframed case. Indeed the four "kinks" of Figure 7 generate a central $\mathbb{Z}$ within long v-knots, and it is not hard to show that the sequence

$$
1 \longrightarrow \mathbb{Z} \longrightarrow\{\text { long v-knots }\} \longrightarrow\{\text { unframed long v-knots }\} \longrightarrow 1
$$

is split and exact. The same can be said for w-knots. 


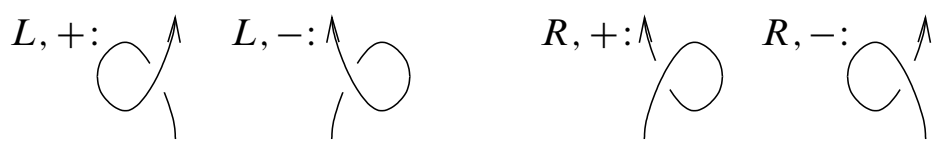

Figure 7: The positive and negative under-then-over kinks (left), and the positive and negative over-then-under kinks (right). In each pair the negative kink is the \#-inverse of the positive kink, where \# denotes the concatenation operation.

Exercise 3.6 Show that a splitting of the sequence (17) is given by the "self-linking" invariant $s l:\{$ long v-knots $\} \rightarrow \mathbb{Z}$ defined by

$$
\operatorname{sl}(K):=\sum_{\substack{\text { crossings } \\ x \text { in } K}} \operatorname{sign} x,
$$

where $K$ is a v-knot diagram, and the sign of a crossing $x$ is defined so as to agree with the signs in Figure 7.

Remark 3.7 Note that w-knots are strictly weaker than v-knots. A notorious example is the Kishino knot (see eg [36]) which is non-trivial as a v-knot, yet both it and its mirror are trivial as w-knots. Yet ordinary knots inject even into w-knots, as the Wirtinger presentation makes sense for w-knots and therefore w-knots have a "fundamental quandle" which generalizes the fundamental quandle of ordinary knots [54], and as the fundamental quandle of ordinary knots separates ordinary knots [50, Corollary 16.3].

3.1.1 A topological construction of Satoh's tubing map Following Satoh [81] and using the same constructions as in Section 2.2.2, we can map w-knots to ("long") ribbon tubes in $\mathbb{R}^{4}$ (and the relations in Figure 6 still hold). It is natural to expect that this "tubing" map is an isomorphism; in other words, that the theory of w-knots provides a "Reidemeister framework" for long ribbon tubes in $\mathbb{R}^{4}$ : that every long ribbon tube is in the image of this map and that two "w-knot diagrams" represent the same long ribbon tube iff they differ by a sequence of moves as in Figure 6. This remains unproven.

Let $\delta:\{\mathrm{v}$-knots $\} \rightarrow\left\{\right.$ Ribbon tori in $\left.\mathbb{R}^{4}\right\}$ denote the tubing map. In Satoh [81], $\delta$ is called "Tube". It is worthwhile giving a completely "topological" definition of $\delta$. To do this we must start with a topological interpretation of v-knots.

The standard topological interpretation of v-knots (see eg [59]) is that they are oriented framed knots drawn ${ }^{23}$ on an oriented surface $\Sigma$, modulo "stabilization", which is the addition and/or removal of empty handles (handles that do not intersect with the knot).

${ }^{23}$ Here and below, "drawn on $\Sigma$ " means "embedded in $\Sigma \times[-\epsilon, \epsilon]$ ". 
We prefer an equivalent, yet even more bare-bones approach. For us, a virtual knot is an oriented framed knot $\gamma$ drawn on a "virtual surface $\Sigma$ for $\gamma$ ". More precisely, $\Sigma$ is an oriented surface that may have a boundary, $\gamma$ is drawn on $\Sigma$, and the pair $(\Sigma, \gamma)$ is taken modulo the following relations:

- Isotopies of $\gamma$ on $\Sigma$ (meaning, in $\Sigma \times[-\epsilon, \epsilon]$ ).

- Tearing and puncturing parts of $\Sigma$ away from $\gamma$ :
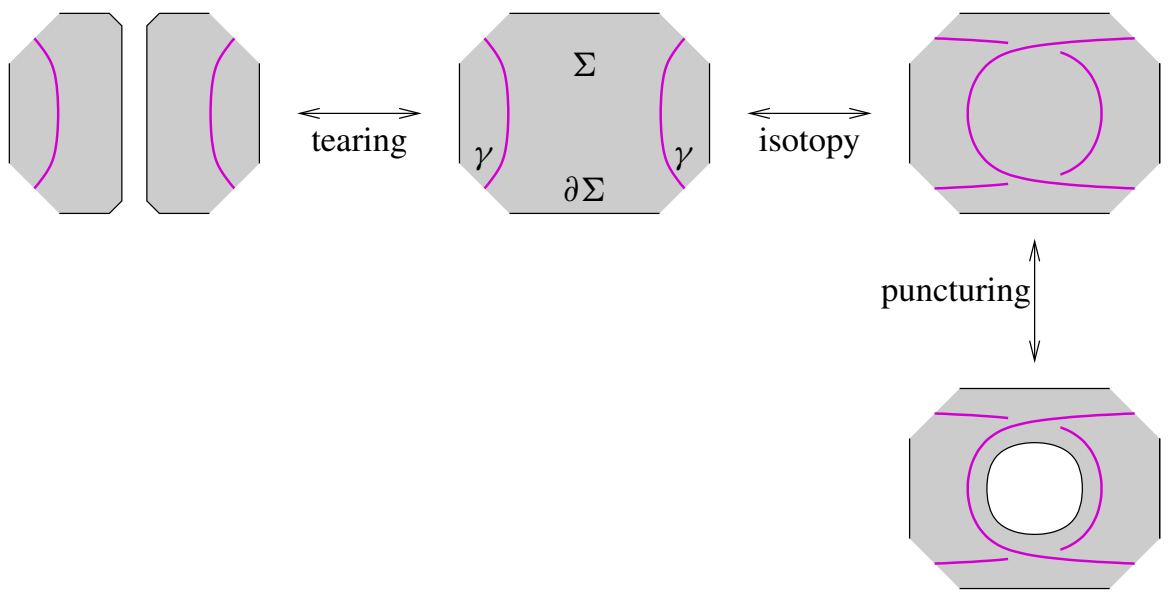

(We call $\Sigma$ a "virtual surface" because tearing and puncturing imply that we only care about it in the immediate vicinity of $\gamma$ ).

We can now define ${ }^{24}$ a map $\delta$, defined on $\mathrm{v}$-knots and taking values in ribbon tori in $\mathbb{R}^{4}$. Given $(\Sigma, \gamma)$, embed $\Sigma$ arbitrarily in $\mathbb{R}_{x z t}^{3} \subset \mathbb{R}^{4}$. Note that the unit normal bundle of $\Sigma$ in $\mathbb{R}^{4}$ is a trivial circle bundle and it has a distinguished trivialization, constructed using its positive $y$-direction section and the orientation that gives each fibre a linking number +1 with the base $\Sigma$. We say that a normal vector to $\Sigma$ in $\mathbb{R}^{4}$ is "near unit" if its norm is between $1-\epsilon$ and $1+\epsilon$. The near-unit normal bundle of $\Sigma$ has as fibre an annulus that can be identified with $[-\epsilon, \epsilon] \times S^{1}$ (identifying the radial direction $[1-\epsilon, 1+\epsilon]$ with $[-\epsilon, \epsilon]$ in an orientation-preserving manner), and hence, the near-unit normal bundle of $\Sigma$ defines an embedding of $\Sigma \times[-\epsilon, \epsilon] \times S^{1}$ into $\mathbb{R}^{4}$. On the other hand, $\gamma$ is embedded in $\Sigma \times[-\epsilon, \epsilon]$ so $\gamma \times S^{1}$ is embedded in $\Sigma \times[-\epsilon, \epsilon] \times S^{1}$, and we can let $\delta(\gamma)$ be the composition

$$
\gamma \times S^{1} \hookrightarrow \Sigma \times[-\epsilon, \epsilon] \times S^{1} \hookrightarrow \mathbb{R}^{4},
$$

which is a torus in $\mathbb{R}^{4}$, oriented using the given orientation of $\gamma$ and the standard orientation of $S^{1}$.

${ }^{24}$ Following a private discussion with Dylan Thurston. 
A framing of a knot (or a v-knot) $\gamma$ can be thought of as a "nearby companion" to $\gamma$. Applying the above procedure to a knot and a nearby companion simultaneously, we find that $\delta$ takes framed v-knots to framed ribbon tori in $\mathbb{R}^{4}$, where a framing of a tube in $\mathbb{R}^{4}$ is a continuous up-to-homotopy choice of unit normal vector at every point of the tube. Note that from the perspective of flying rings as in Section 2.2.1, a framing is a "companion ring" to a flying ring. In the framing of $\delta(\gamma)$ the companion ring is never linked with the main ring, but can fly parallel inside, outside, above or below it and change these positions, as shown in Figure 8.

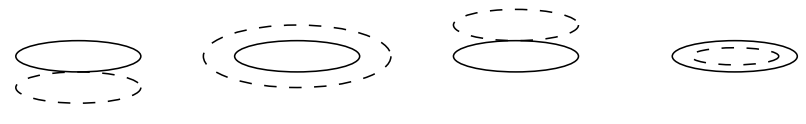

Figure 8: Framing as companion rings.

We leave it to the reader to verify that $\delta(\gamma)$ is ribbon, that it is independent of the choices made within its construction, that it is invariant under isotopies of $\gamma$ and under tearing and puncturing of $\Sigma$, that it is also invariant under the OC relation of Figure 6 and hence, the true domain of $\delta$ is w-knots, and that it is equivalent to Satoh's tubing map.

\subsection{Finite type invariants of v-knots and w-knots}

Much as for v-braids and w-braids (see Section 2.3) and much as for ordinary knots (see eg [6]) we define finite-type invariants for v-knots and for w-knots using an alternation scheme with $\bar{x} \rightarrow \bar{X} \bar{X}$ and $\bar{x} \rightarrow \bar{X}-\lambda$. That is, given any invariant of $\mathrm{v}$ - or $\mathrm{w}$-knots taking values in an abelian group, we extend the invariant to v- or w-knots also containing "semi-virtual crossings" like " $\mathrm{k}$ and using the above assignments, and we declare an invariant to be "of type $m$ " if it vanishes on v- or w-knots with more than $m$ semi-virtuals. As for v- and w-braids and as for ordinary knots, such invariants have an " $m^{\text {th }}$ derivative", their "weight system", which is a linear functional on the space $\mathcal{A}^{s v}(\uparrow)$ (for v-knots) or $\mathcal{A}^{s w}(\uparrow)$ (for w-knots). We turn to the definitions of these spaces, following $[42 ; 20]$.

Definition 3.8 An "arrow diagram" is a chord diagram along a long line (called "the skeleton"), in which the chords are oriented (hence "arrows"). An example is given in Figure 9. Let $\mathcal{D}^{v}(\uparrow)$ be the space of formal linear combinations of arrow diagrams. Let $\mathcal{A}^{v}(\uparrow)$ be $\mathcal{D}^{v}(\uparrow)$ modulo all "6T relations". Here a $6 \mathrm{~T}$ relation is any (signed) combination of arrow diagrams obtained from the diagrams in Figure 3 by placing the 3 vertical strands there along a long line skeleton in any order, and possibly adding some 
further arrows in between, as shown in Figure 9. Let $\mathcal{A}^{s v}(\uparrow)$ be the further quotient of $\mathcal{A}^{v}(\uparrow)$ by the RI relation, where the RI (for rotation number independence) relation asserts that an isolated arrow pointing to the right equals an isolated arrow pointing to the left, ${ }^{25}$ as shown in Figure 9.
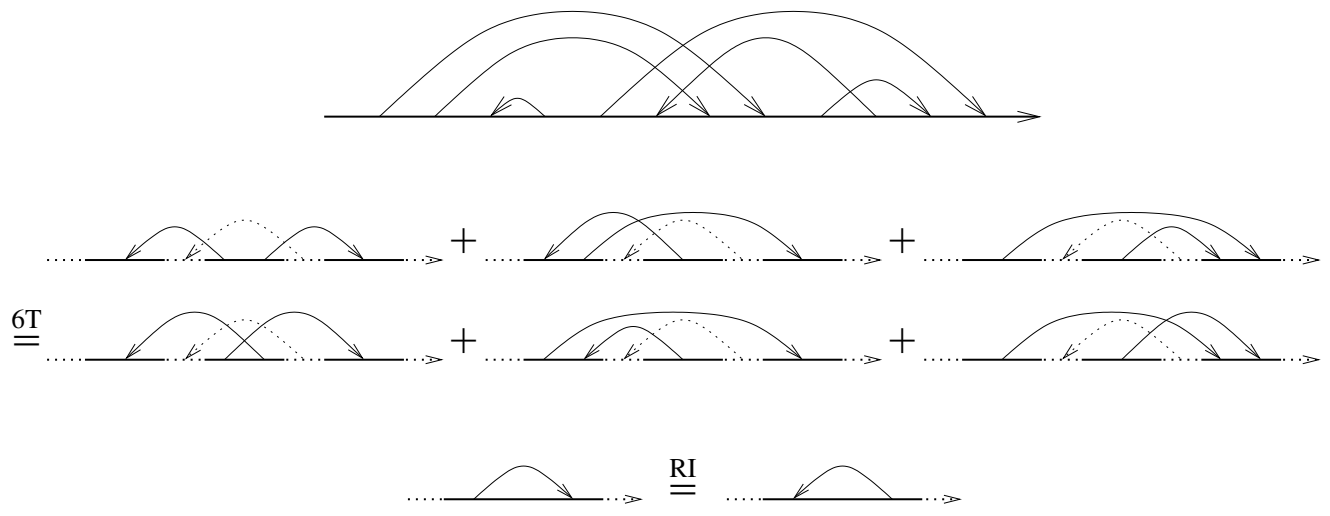

Figure 9: An arrow diagram of degree 6, a 6T relation, and an RI relation. The dotted parts indicate that there may be more arrows on other parts of the skeleton, however these remain the same throughout the relation.

Let $\mathcal{A}^{w}(\uparrow)$ be the further quotient of $\mathcal{A}^{v}(\uparrow)$ by the TC relation, first displayed in Figure 4 and reproduced for the case of a long line skeleton in Figure 10. Likewise, let $\mathcal{A}^{s w}(\uparrow):=\mathcal{A}^{s v}(\uparrow) / \mathrm{TC}=\mathcal{A}^{w}(\uparrow) / \mathrm{RI}$. Alternatively, noting that given TC two of the terms in 6T drop out, $\mathcal{A}^{w}(\uparrow)$ is seen to be the space of formal linear combinations of arrow diagrams modulo the TC and $\overrightarrow{4 T}$ relations, displayed in Figures 4 and 10 . Likewise, $\mathcal{A}^{s w}=\mathcal{D}^{v} / \mathrm{TC}, \overrightarrow{4 \mathrm{~T}}$, RI. Finally, grade $\mathcal{D}^{v}(\uparrow)$ and all of its quotients by declaring that the degree of an arrow diagram is the number of arrows in it.

As an example, the spaces $\mathcal{A}^{v, s v, w, s w}(\uparrow)$ (that is, any of the spaces above) restricted to degrees up to 2 are studied in detail in Section 4.3.

In the same manner as in the theory of finite-type invariants of ordinary knots (see especially [6, Section 3]), the spaces $\mathcal{A}^{\star}(\uparrow)$ (meaning, all of the spaces above) carry much algebraic structure. The juxtaposition product makes them into graded algebras. The product of two finite-type invariants is a finite-type invariant (whose type is the sum of the types of the factors); this induces a product on weight systems, and therefore a co-product $\Delta$ on arrow diagrams. In brief (and much the same as in the usual finite-type story), the co-product $\Delta D$ of an arrow diagram $D$ is the sum of all ways of dividing the arrows in $D$ between a "left co-factor" and a "right co-factor". In summary:

25 The XII relation of [20] follows from RI and need not be imposed. 


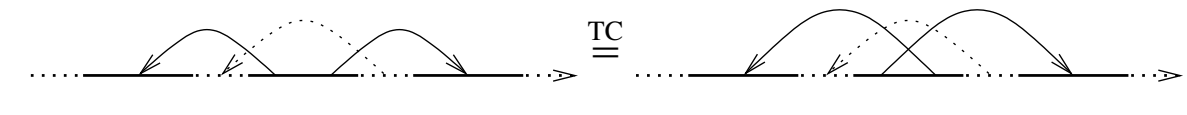

and

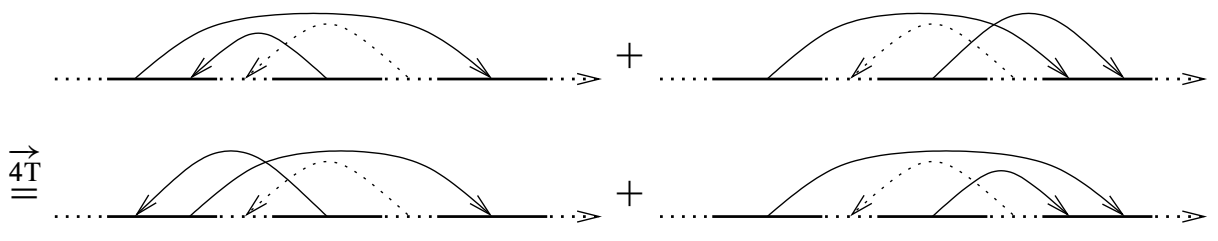

Figure 10: The TC and the $\overrightarrow{4 \mathrm{~T}}$ relations for knots.

Proposition 3.9 $\mathcal{A}^{v}(\uparrow), \mathcal{A}^{s v}(\uparrow), \mathcal{A}^{w}(\uparrow)$, and $\mathcal{A}^{s w}(\uparrow)$ are co-commutative graded bialgebras.

By the Milnor-Moore theorem [75, Theorem 6.11] we find that $\mathcal{A}^{v, s v, w, s w}(\uparrow)$ are the universal enveloping algebras of their Lie algebras of primitive elements (that is, elements $D$ such that $\Delta(D)=1 \otimes D+D \otimes 1$ ). Denote these (graded) Lie algebras by $\mathcal{P}^{v, s v, w, s w}(\uparrow)$, respectively.

When we grow up we'd like to understand $\mathcal{A}^{v}(\uparrow)$ and $\mathcal{A}^{s v}(\uparrow)$. At the moment we know only very little about these spaces beyond the generalities of Proposition 3.9. In Section 4.1 some dimensions of low degree parts of $\mathcal{A}^{v, s v}(\uparrow)$ are discussed. Also, given a finite-dimensional Lie bialgebra and a finite-dimensional representation thereof, we know how to construct linear functionals on $\mathcal{A}^{v}(\uparrow)$ (one in each degree $[49 ; 70]$ ), but not on $\mathcal{A}^{s v}(\uparrow)$. But we don't even know which degree $m$ linear functionals on $\mathcal{A}^{s v}(\uparrow)$ are the weight systems of degree $m$ invariants of v-knots (that is, we have not solved the "Fundamental Problem" [23] for v-knots).

As we shall see below, the situation is much brighter for $\mathcal{A}^{w, s w}(\uparrow)$.

\subsection{Expansions for w-knots}

The notion of "an expansion" (or "a universal finite-type invariant") for w-knots (or $\mathrm{v}$-knots) is defined in complete analogy with the parallel notion for usual knots (see eg [6]), except replacing double points $\bar{X}$ with semi-virtual crossings $" \alpha$ and $\bar{\alpha}$, and replacing chord diagrams by arrow diagrams. Alternatively, it is the same as an expansion for w-braids (as in Definition 2.11), simply replacing w-braids by w-knots. Just as in the cases of u-knots (ie ordinary knots) and/or w-braids, the existence of an expansion $Z:\{$ w-knots $\} \rightarrow \mathcal{A}^{s w}(\uparrow)$ is equivalent to the statement "every weight system integrates", ie "every degree $m$ linear functional on $\mathcal{A}^{s w}(\uparrow)$ is the $m^{\text {th }}$ derivative of a type $m$ invariant of long w-knots". 
Theorem 3.10 There exists an expansion $Z:\{w-k n o t s\} \rightarrow \mathcal{A}^{s w}(\uparrow)$.

Proof It is best to define $Z$ by an example, and it is best to display the example only as a picture:

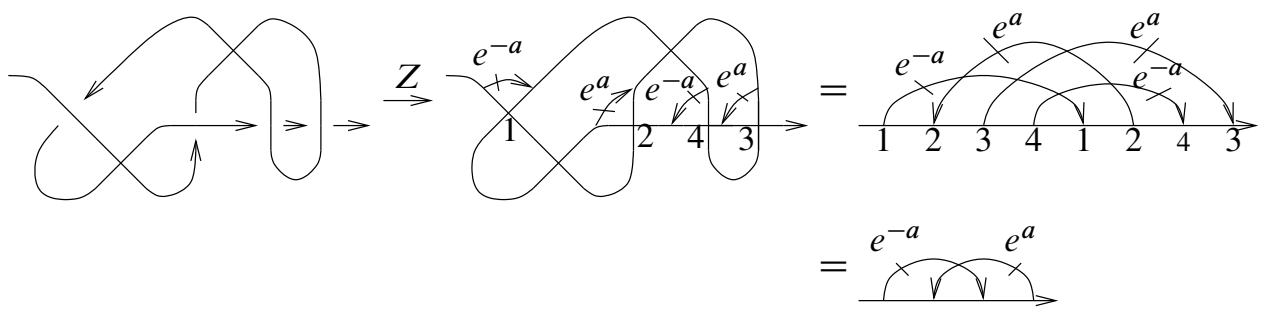

It is clear how to define $Z(K)$ in the general case: for every crossing in $K$ place an exponential reservoir of arrows (compare with Equation (15)) next to that crossing, with the arrows heading from the upper strand to the lower strand, taking positive reservoirs ( $e^{a}$, with $a$ symbolizing the arrow) for positive crossings and negative reservoirs $\left(e^{-a}\right)$ for negative crossings, and then tug the skeleton until it looks like a straight line. Note that the TC relation in $\mathcal{A}^{s w}$ is used to show that all reasonable ways of placing an arrow reservoir at a crossing (with its heading and sign fixed) are equivalent:

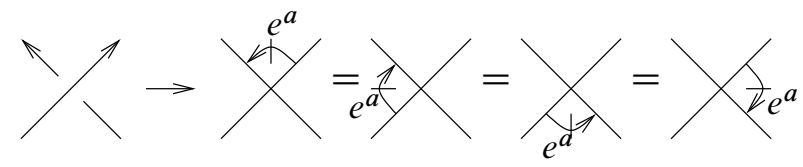

The same proof that shows the invariance of $Z$ in the braid case (see Theorem 2.15) works here as well, ${ }^{26}$ and the same argument as in the braid case shows the universality of $Z$.

Remark 3.11 Using the language of Gauss diagrams (Remark 3.4) the definition of $Z$ is even simpler. Simply map every positive arrow in a Gauss diagram to a positive $\left(e^{a}\right)$ reservoir, and every negative one to a negative $\left(e^{-a}\right)$ reservoir:
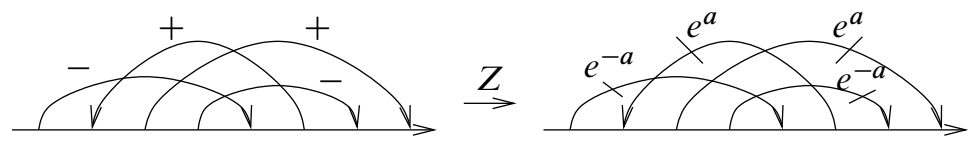

An expansion (a universal finite-type invariant) is as interesting as its target space, for it is just a tool that takes linear functionals on the target space to finite-type invariants on its domain space. The purpose of the next section is to find out how interesting our present target space, $\mathcal{A}^{s w}(\uparrow)$, and its "parent", $\mathcal{A}^{w}(\uparrow)$, are.

${ }^{26} \mathrm{~A}$ tiny bit of extra care is required for invariance under $\mathrm{R} 1^{s}$ : it easily follows from RI. 


\subsection{Jacobi diagrams, trees and wheels}

In studying $\mathcal{A}^{w}(\uparrow)$ we again follow the model set by usual knots: we introduce the space $\mathcal{A}^{w t}$ of "w-Jacobi diagrams" and show that it is isomorphic to $\mathcal{A}^{w}$. Major advantages of working with $\mathcal{A}^{w t}$ are that the co-product, the primitives, and the relationship with Lie algebras are much more natural and easy to describe. Compare the following definitions and theorem with [6, Section 3].

Definition 3.12 A "w-Jacobi diagram on a long line skeleton"27 is a connected graph made of the following ingredients:

- A "long" oriented "skeleton" line. We usually draw the skeleton line a bit thicker for emphasis.

- Other directed edges, usually called "arrows".

- Trivalent "skeleton vertices" in which an arrow starts or ends on the skeleton line.

- Trivalent "internal vertices" in which two arrows end and one arrow begins (this will be important in Section 3.5 where we relate these diagrams to Lie algebras). The internal vertices are "oriented": of the two arrows that end in an internal vertices, one is marked as "left" and the other is marked as "right". In reality when a diagram is drawn in the plane, we almost never mark "left" and "right", but instead assume the "left" and "right" inherited from the plane, as seen from the outgoing arrow from the given vertex.

Note that we allow multiple arrows connecting the same two vertices (though at most two are possible, given connectedness and trivalence) and we allow "bubbles": arrows that begin and end in the same vertex. Also keep in mind that for the purpose of determining equality of diagrams the skeleton line is distinguished. The "degree" of a w-Jacobi diagram is half the number of trivalent vertices in it, including both internal and skeleton vertices. An example of a w-Jacobi diagram is in Figure 11.

Definition 3.13 Let $\mathcal{D}^{w t}(\uparrow)$ be the graded vector space of formal linear combinations $^{28}$ of w-Jacobi diagrams on a long line skeleton, and let $\mathcal{A}^{w t}(\uparrow)$ be $\mathcal{D}^{w t}(\uparrow)$ modulo the $\overrightarrow{\mathrm{STU}}_{1}, \overrightarrow{\mathrm{STU}}_{2}$, and TC relations of Figure 12. Note that each diagram appearing in each $\overrightarrow{\text { STU }}$ relation has a "central edge" $e$ which can serve as an "identifying name" for that $\overrightarrow{\mathrm{STU}}$. Thus, given a diagram $D$ with a marked edge $e$ which is either on the

${ }^{27}$ What a mouthful! We usually short this to "w-Jacobi diagram", or sometimes "arrow diagram" or just "diagram".

$28 \mathbb{Q}$-linear, or any other field of characteristic 0. 


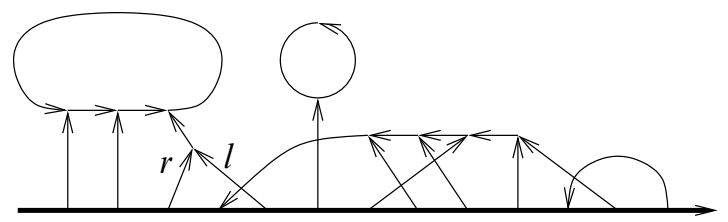

Figure 11: A degree $11 \mathrm{w}-J a c o b i$ diagram on a long line skeleton. It has a skeleton line at the bottom, 13 vertices along the skeleton (of which 2 are incoming and 11 are outgoing), 9 internal vertices (with only one explicitly marked with "left" $(l)$ and "right" $(r))$ and one bubble. The five quadrivalent vertices that seem to appear in the diagram are just projection artifacts and graph-theoretically, they don't exist.

skeleton or which contacts the skeleton, there is an unambiguous $\overrightarrow{\text { STU }}$ relation "around" or "along" the edge $e$.
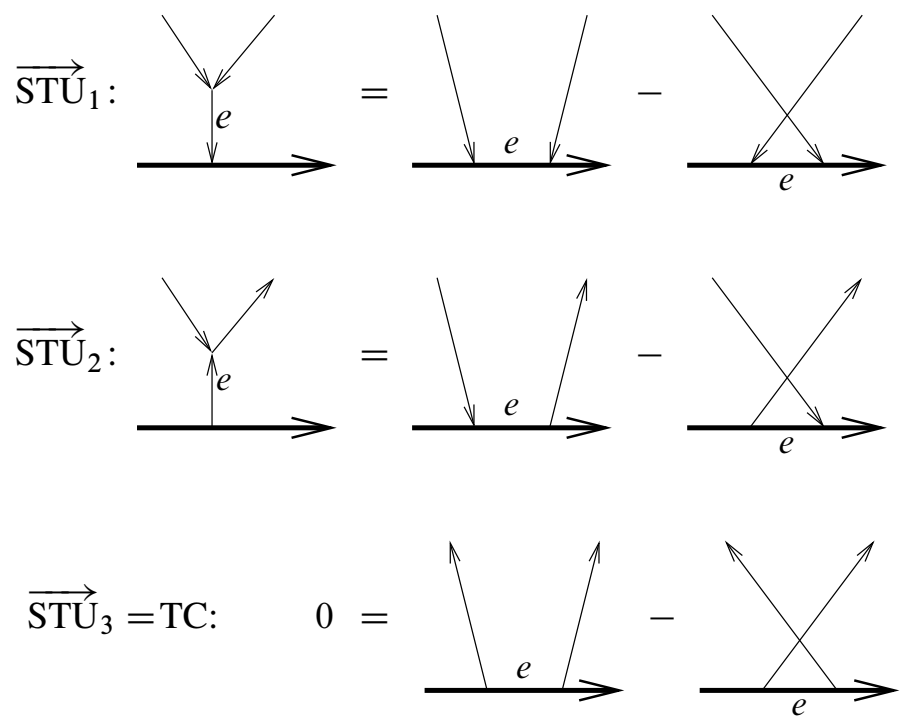

Figure 12: The $\overrightarrow{\mathrm{STU}}_{1,2}$ and TC relations with their "central edges" marked $e$.

We like to call the following theorem "the bracket-rise theorem", for it justifies the introduction of internal vertices, and as should be clear from the $\overrightarrow{\text { STU }}$ relations and as will become even clearer in Section 3.5, internal vertices can be viewed as "brackets". Two other bracket-rise theorems are [6, Theorem 6] and Ohtsuki's theorem, ie [78, Theorem 4.9]. 
Theorem 3.14 (Bracket-rise) The obvious inclusion $\iota: \mathcal{D}^{v}(\uparrow) \rightarrow \mathcal{D}^{w t}(\uparrow)$ of arrow diagrams (see Definition 3.8) into w-Jacobi diagrams descends to the quotient $\mathcal{A}^{w}(\uparrow)$ and induces an isomorphism ${ }^{29}$

$$
\overline{l:} \mathcal{A}^{w}(\uparrow) \stackrel{\sim}{\longrightarrow} \mathcal{A}^{w t}(\uparrow) .
$$

Furthermore, the $\overrightarrow{\mathrm{AS}}$ and $\overrightarrow{\mathrm{IHX}}$ relations of Figure 13 hold in $\mathcal{A}^{w t}(\uparrow)$.

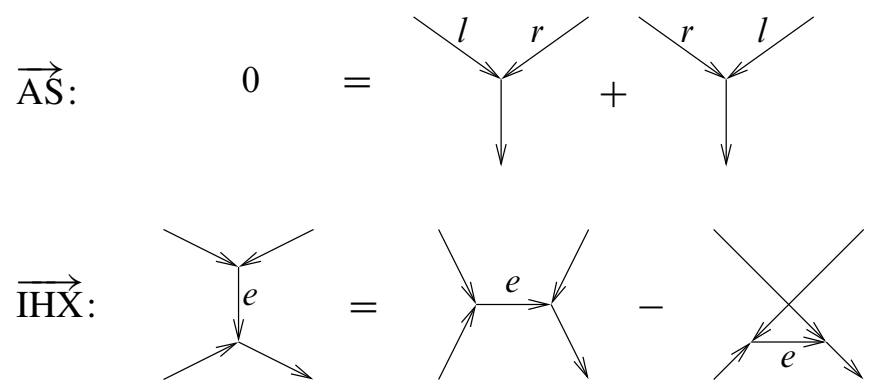

Figure 13: The $\overrightarrow{\mathrm{AS}}$ and $\overrightarrow{\mathrm{IHX}}$ relations.

Proof The proof, joint with D Thurston, is modelled after the proof of [6, Theorem 6]. To show that $\iota$ descends to $\mathcal{A}^{w}(\uparrow)$ we just need to show that in $\mathcal{A}^{w t}(\uparrow), \overrightarrow{4 \mathrm{~T}}$ follows from $\overrightarrow{\mathrm{STU}}_{1,2}$. Indeed, applying $\overrightarrow{\mathrm{STU}}_{1}$ along the edge $e_{1}$ and $\overrightarrow{\mathrm{STU}}_{2}$ along $e_{2}$ in the picture below, we get the two sides of $\overrightarrow{4 T}$ :

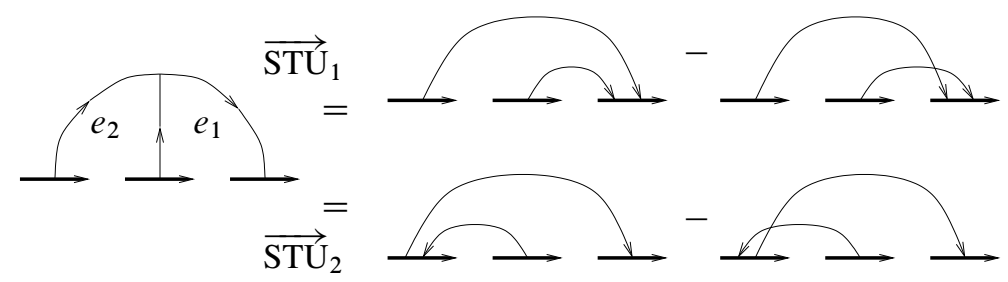

The fact that $\tau$ is surjective is easy: indeed, for diagrams in $\mathcal{A}^{w t}(\uparrow)$ that have no internal vertices there is nothing to show, for they are really in $\mathcal{A}^{w}(\uparrow)$. Further, by repeated use of $\overrightarrow{\mathrm{STU}}_{1,2}$ relations, all internal vertices in any diagram in $\mathcal{A}^{w t}(\uparrow)$ can be removed (remember that the diagrams in $\mathcal{A}^{w t}(\uparrow)$ are always connected, and in particular, if they have an internal vertex they must have an internal vertex connected by an edge to the long line skeleton, and the latter vertex can be removed first).

To complete the proof that $\bar{l}$ is an isomorphism it is enough to show that the "elimination of internal vertices" procedure of the last paragraph is well-defined: that its output is

${ }^{29}$ At this point a vector space isomorphism, but we'll soon define a bialgebra structure on $\mathcal{A}^{\text {wt }}$ to make it into an isomorphism of bialgebras. 
independent of the order in which $\overrightarrow{\mathrm{STU}}_{1,2}$ relations are applied in order to eliminate internal vertices. Indeed, this done, the elimination map would by definition satisfy the $\overrightarrow{\mathrm{STU}}_{1,2}$ relations, and thus descend to a well-defined inverse for $\bar{\imath}$.

On diagrams with just one internal vertex, Equation (18) shows that all ways of eliminating that vertex are equivalent modulo $\overrightarrow{4 T}$ relations, and hence, the elimination map is well-defined on such diagrams.

We proceed by induction on the number of internal vertices. We have shown that $\tau$ is well defined if there is only one internal vertex. Now assume that we have shown that the elimination map is well defined on all diagrams with at most $k$ internal vertices for some positive integer $k \geq 1$, and let $D$ be a diagram with $(k+1)$ internal vertices. Let $e$ and $e^{\prime}$ be edges in $D$ that connect the skeleton of $D$ to an internal vertex. We need to show that any elimination process that begins with eliminating $e$ yields the same answer, modulo $\overrightarrow{4 T}$, as any elimination process that begins with eliminating $e^{\prime}$. There are several cases to consider.

Case I The edges $e$ and $e^{\prime}$ connect the skeleton to different internal vertices of $D$ :

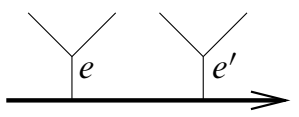

In this case, after eliminating $e$ we get a signed sum of two diagrams with exactly 7 internal vertices, and since the elimination process is well-defined on such diagrams, we may as well continue by eliminating $e^{\prime}$ in each of those, getting a signed sum of 4 diagrams with 6 internal vertices each. On the other hand, if we start by eliminating $e^{\prime}$ we can continue by eliminating $e$, and we get the same signed sum of 4 diagrams with 6 internal vertices.

Case II The edges $e$ and $e^{\prime}$ are connected to the same internal vertex $v$ of $D$, yet some other edge $e^{\prime \prime}$ exists in $D$ that connects the skeleton of $D$ to some other internal vertex $v^{\prime}$ in $D$ :

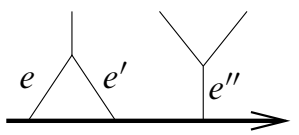

In that case, use the previous case and the transitivity of equality: (elimination starting with $e)=\left(\right.$ elimination starting with $\left.e^{\prime \prime}\right)=\left(\right.$ elimination starting with $\left.e^{\prime}\right)$.

Case III This is what remains if neither Case I nor Case II hold. In that case, $D$ must have a schematic form as below, with the "blob" not connected to the skeleton other 
than via $e$ or $e^{\prime}$, yet further arrows may exist outside of the blob:

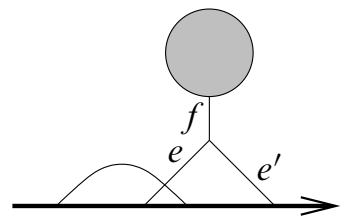

Let $f$ denote the edge connecting the blob to $e$ and $e^{\prime}$. The "two in one out" rule for vertices implies that any part of a diagram must have an excess of incoming edges over outgoing edges, equal to the total number of vertices in that diagram part. Applying this principle to the blob, we find that it must contain exactly one vertex, as shown below. Then by the "two in one out" rule $f$ must be oriented upwards, and hence, by the "two in one out" rule again, $e$ and $e^{\prime}$ must be oriented upwards as well.

We leave it to the reader to verify that in this case the two ways of applying the elimination procedure, $e$ and then $f$ or $e^{\prime}$ and then $f$, yield the same answer modulo $\overrightarrow{4 \mathrm{~T}}$ (in fact, that answer is 0 ):

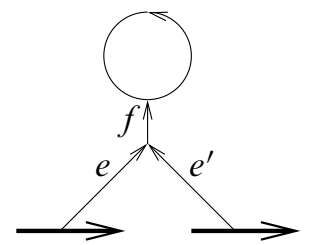

We also leave it to the reader to verify that $\overrightarrow{\mathrm{STU}}_{1}$ implies $\overrightarrow{\mathrm{AS}}$ and $\overrightarrow{\mathrm{IHX}}$. In Section 3.5 we'll describe the relationship between $\mathcal{A}^{w t}$ and Lie algebras. Algebraically, the relations $\overrightarrow{\mathrm{STU}}_{1}, \overrightarrow{\mathrm{AS}}$ and $\overrightarrow{\mathrm{IHX}}$ are restatements of the anti-symmetry of the bracket and of Jacobi's identity: if $[x, y]:=x y-y x$, then $0=[x, y]+[y, x]$ and $[x,[y, z]]=$ $[[x, y], z]-[[x, z], y]$.

Note that $\mathcal{A}^{w t}(\uparrow)$ inherits algebraic structure from $\mathcal{A}^{w}(\uparrow)$ : it is an algebra by concatenation of diagrams, and a co-algebra with $\Delta(D)$, for $D \in \mathcal{D}^{w t}(\uparrow)$, being the sum of all ways of dividing $D$ between a "left co-factor" and a "right co-factor" so that connected components of $D-S$ are kept intact, where $S$ is the skeleton line of $D$ (compare with [6, Definition 3.7]).

As $\mathcal{A}^{w}(\uparrow)$ and $\mathcal{A}^{w t}(\uparrow)$ are canonically isomorphic, from this point on we will not keep the distinction between the two spaces. One may add the RI relation to the definition of $\mathcal{A}^{w t}(\uparrow)$ to get a space $\mathcal{A}^{s w t}(\uparrow)$. For an unframed version one may add the stronger framing independence (FI) relation, setting $D_{L}=D_{R}=0$, with $D_{L}$ and $D_{R}$ the single arrows as in Figure 14. The resulting space is called $\mathcal{A}^{r w t}(\uparrow)$. The 
statement and proof of the bracket rise theorem adapt with no difficulty, and we find that $\mathcal{A}^{s w}(\uparrow) \cong \mathcal{A}^{s w t}(\uparrow)$ and $\mathcal{A}^{r w}(\uparrow) \cong \mathcal{A}^{r w t}(\uparrow)$. In the future we'll drop the $t$ from all superscripts.

The advantages of allowing internal trivalent vertices are already apparent (for example, note that there is a nice description of primitive elements: they are the arrow diagrams which remain connected if the skeleton is removed). Further advantages will emerge in Section 3.5 .
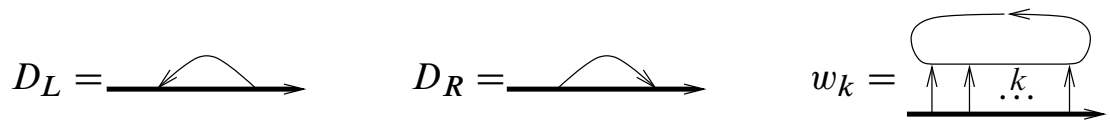

Figure 14: The left-arrow diagram $D_{L}$, the right-arrow diagram $D_{R}$ and the $k$-wheel $w_{k}$.

Theorem 3.15 The bialgebra $\mathcal{A}^{w}(\uparrow)$ is the bialgebra of polynomials in the diagrams $D_{L}, D_{R}$ and $w_{k}$ (for $k \geq 1$ ) shown in Figure 14 , where $\operatorname{deg} D_{L}=\operatorname{deg} D_{R}=1$ and $\operatorname{deg} w_{k}=k$, subject to the one relation $w_{1}=D_{L}-D_{R}$. Thus, $\mathcal{A}^{w}(\uparrow)$ has two generators in degree 1 and one generator in every degree greater than 1 , as stated in Section 4.1.

Sketch of proof Readers familiar with the diagrammatic PBW theorem [6, Theorem 8] will note that it has a direct analogue for the $\mathcal{A}^{w}(\uparrow)$ case, and that the proof in [6] carries through almost verbatim. Namely, the space $\mathcal{A}^{w}(\uparrow)$ is isomorphic to a space $\mathcal{B}^{w}$ of "unitrivalent diagrams" with symmetrized univalent ends modulo $\overrightarrow{\mathrm{AS}}$ and $\overrightarrow{\mathrm{IHX}}$. Given the "two in one out" rule for arrow diagrams in $\mathcal{A}^{w}(\uparrow)$ (and hence, in $\mathcal{B}^{w}$ ) the connected components of diagrams in $\mathcal{B}^{w}$ can only be "trees" or "wheels". A tree is a unitrivalent diagram with no cycles (oriented or not). A wheel is a single oriented cycle with some number of incoming "spokes" (see $w_{k}$ in Figure 14 and remove the skeleton line). The reader might object that there are also "wheels of trees" — trees attached to an oriented cycle — but these can be reduced to linear combinations of wheels using the $\overrightarrow{\mathrm{IHX}}$ relation.

Trees vanish if they have more than one leaf, as their leafs are symmetric while their internal vertices are anti-symmetric, so $\mathcal{B}^{w}$ is generated by wheels and by the one-leafone-root tree, which is simply a single arrow. Wheels map to the $w_{k}$ in $\mathcal{A}^{w}(\uparrow)$ under the isomorphism, and the arrow maps to the average of $D_{L}$ and $D_{R}$. The relation $w_{1}=D_{L}-D_{R}$ is then easily verified using $\overrightarrow{\mathrm{STU}}_{2}$.

One may also argue directly, without using $\mathcal{B}^{w}$. In short, let $D$ be a diagram in $\mathcal{A}^{w}(\uparrow)$ and $S$ is its skeleton. Then $D-S$ may have several connected components, whose 
"legs" are intermingled along $S$. Using the $\overrightarrow{\text { STU }}$ relations these legs can be sorted (at a cost of diagrams with fewer connected components, which could have been treated earlier in an inductive proof). At the end of the sorting procedure one can see that the only diagrams that remain are our declared generators. It remains to show that our generators are linearly independent (apart from the relation $w_{1}=D_{L}-D_{R}$ ). For the generators in degree 1 , simply write everything out explicitly in the spirit of Section 4.3.2. In higher degrees there is only one primitive diagram in each degree, so it is enough to show that $w_{k} \neq 0$ for every $k$. This can be done "by hand", but it is more easily done using Lie algebraic tools in Section 3.5.

Exercise 3.16 Show that the bialgebra $\mathcal{A}^{r w}(\uparrow)$ (see Section 4.1) is the bialgebra of polynomials in the wheel diagrams $w_{k}$ for $k \geq 2$, and that $\mathcal{A}^{s w}(\uparrow)$ is the bialgebra of polynomials in the same wheel diagrams and an additional generator $D_{A}:=D_{L}=D_{R}$.

Proposition 3.17 In $\mathcal{A}^{w}(\bigcirc)$ all wheels vanish, and hence the bialgebra $\mathcal{A}^{w}(\bigcirc)$ is the bialgebra of polynomials in a single variable $D_{L}=D_{R}$.

Proof This is [76, Lemma 2.7]. In short, a wheel in $\mathcal{A}^{w}(\bigcirc)$ can be reduced using $\overrightarrow{\mathrm{STU}}_{2}$ to a difference of trees, as shown in Figure 15. One of these trees has two adjoining leafs, hence it is 0 by TC and $\overrightarrow{\mathrm{AS}}$. In the other, two of the leafs can be commuted "around the circle" using TC until they are adjoining and hence vanish by TC and $\overrightarrow{\mathrm{AS}}$.

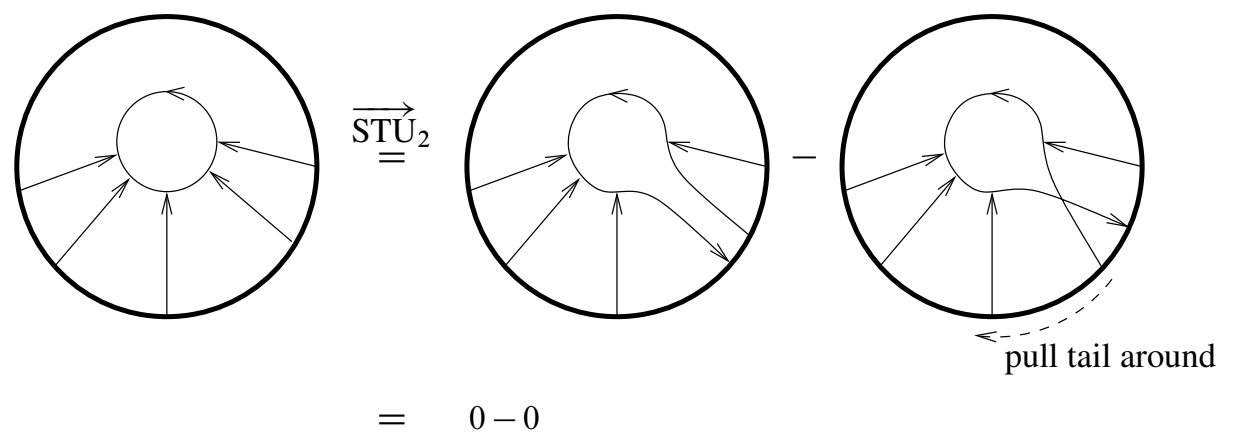

Figure 15: Wheels in a circle vanish.

Exercise 3.18 Show that $\mathcal{A}^{s w}(\bigcirc) \cong \mathcal{A}^{w}(\bigcirc)$ and yet $\mathcal{A}^{r w}(\bigcirc)$ vanishes except in degree 0 . 
The following two exercises may help the reader to develop a better "feel" for $\mathcal{A}^{w}(\uparrow)$ and will be needed within the discussion of the Alexander polynomial (especially within Definition 3.31).

Exercise 3.19 Show that the "commutators commute" (CC) relation, shown below, holds in $\mathcal{A}^{w}(\uparrow)$ :

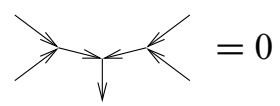

(Interpreted in terms of Lie algebras as in the next section, this relation becomes $[[x, y],[z, w]]=0$, hence the name "commutators commute"). Note that the proof of $\mathrm{CC}$ depends on the skeleton having a single component; later, when we will work with $\mathcal{A}^{w}$-spaces with more complicated skeleta, the CC relation will not hold.

Exercise 3.20 Show that "detached wheels" and "hairy $Y$ s" make sense in $\mathcal{A}^{w}(\uparrow)$. As pictured below, a detached wheel is a wheel with a number of spokes, and a hairy $Y$ is a combinatorial $Y$ shape (three arrows meeting at a single internal vertex) with further "hair" on its trunk (its outgoing arrow):

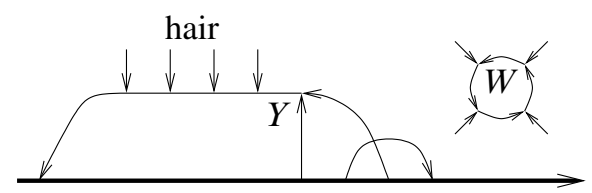

It is specified where the trunk and the leafs of the $Y$ connect to the skeleton, but it is not specified where the spokes of the wheel and where the hair on the $Y$ connect to the skeleton. The content of the exercise is to show that modulo the relations of $\mathcal{A}^{w}(\uparrow)$, it is not necessary to specify this further information: all ways of connecting the spokes and the hair to the skeleton are equivalent. Like the previous exercise, this result depends on the skeleton having a single component.

Remark 3.21 In the case of usual knots and usual chord diagrams, Jacobi diagrams have a topological interpretation using the Goussarov-Habiro calculus of claspers [41; 46]. In the w case a similar such calculus was developed by Watanabe in [84]. Various related results are in $[47 ; 48]$.

\subsection{The relation with Lie algebras}

The theory of finite-type invariants of knots is related to the theory of metrized Lie algebras via the space $\mathcal{A}$ of chord diagrams, as explained in [6, Theorem 4 and Exercise 5.1]. In a similar manner the theory of finite-type invariants of w-knots 
is related to arbitrary finite-dimensional Lie algebras (or equivalently, to doubles of co-commutative Lie bialgebras, as explained below) via the space $\mathcal{A}^{w}(\uparrow)$ of arrow diagrams.

3.5.1 Preliminaries Given a finite-dimensional Lie algebra ${ }^{30} \mathfrak{g}$ let $I \mathfrak{g}:=\mathfrak{g}^{*} \rtimes \mathfrak{g}$ be the semi-direct product of the dual $\mathfrak{g}^{*}$ of $\mathfrak{g}$ with $\mathfrak{g}$, with $\mathfrak{g}^{*}$ taken as an abelian algebra and with $\mathfrak{g}$ acting on $\mathfrak{g}^{*}$ by the usual coadjoint action. In formulae,

$$
\begin{aligned}
I \mathfrak{g} & =\left\{(\varphi, x): \varphi \in \mathfrak{g}^{*}, x \in \mathfrak{g}\right\}, \\
{\left[\left(\varphi_{1}, x_{1}\right),\left(\varphi_{2}, x_{2}\right)\right] } & =\left(x_{1} \varphi_{2}-x_{2} \varphi_{1},\left[x_{1}, x_{2}\right]\right) .
\end{aligned}
$$

In the case where $\mathfrak{g}$ is the algebra $\mathfrak{s o}(3)$ of infinitesimal symmetries of $\mathbb{R}^{3}$, its dual $\mathfrak{g}^{*}$ is $\mathbb{R}^{3}$ itself with the usual action of $\mathfrak{s o ( 3 )}$ on it, and $I \mathfrak{g}$ is the algebra $\mathbb{R}^{3} \rtimes \mathfrak{s o ( 3 )}$ of infinitesimal affine isometries of $\mathbb{R}^{3}$. This is the Lie algebra of the Euclidean group of isometries of $\mathbb{R}^{3}$, which is often denoted $\operatorname{ISO}(3)$. This explains our choice of the name $I \mathfrak{g}$.

Note that if $\mathfrak{g}$ is a co-commutative Lie bialgebra, then $I \mathfrak{g}$ is the "double" of $\mathfrak{g}$ [33]. This is a significant observation, for it is a part of the relationship between this paper and the Etingof-Kazhdan theory of quantization of Lie bialgebras [38]. Yet we will make no explicit use of this observation below.

In the construction that follows we are going to define a map from $\mathcal{A}^{w}$ to $\mathcal{U}(I \mathfrak{g})$, the universal enveloping algebra of $I \mathfrak{g}$. Note that a map $\mathcal{A}^{w} \rightarrow \mathcal{U}(I \mathfrak{g})$ is "almost the same" as a map $\mathcal{A}^{s w} \rightarrow \mathcal{U}(I \mathfrak{g})$, in the following sense. The quotient map $p: \mathcal{A}^{w} \rightarrow \mathcal{A}^{s w}$ has a one-sided inverse $F: \mathcal{A}^{s w} \rightarrow \mathcal{A}^{w}$ defined by

$$
F(D)=\sum_{k=0}^{\infty} \frac{(-1)^{k}}{k !} S_{L}^{k}(D) \cdot w_{1}^{k} .
$$

Here $S_{L}$ denotes the map that sends an arrow diagram to the sum of all ways of deleting a left-going arrow, $S_{L}^{k}$ is $S_{L}$ applied $k$ times, and $w_{1}$ denotes the 1 -wheel, as shown in Figure 14. The reader can verify that $F$ is well-defined, an algebra- and co-algebra homomorphism, and that $p \circ F=\mathrm{id}_{\mathcal{A}^{s w}}$.

3.5.2 The construction Fixing a finite-dimensional Lie algebra $\mathfrak{g}$, we construct a map

$$
\mathcal{T}_{\mathfrak{g}}^{w}: \mathcal{A}^{w} \rightarrow \mathcal{U}(I \mathfrak{g})
$$

${ }^{30}$ Over $\mathbb{Q}$, or another field of characteristic 0 . 
which assigns to every arrow diagram $D$ an element of the universal enveloping algebra $\mathcal{U}(I \mathfrak{g})$. As is often the case in our subject, a picture of a typical example is worth more than a formal definition:

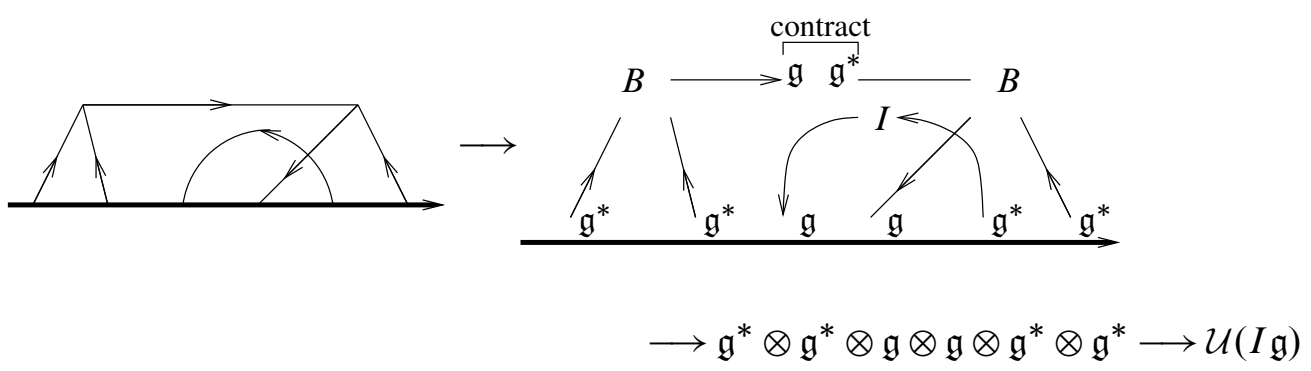

In short, we break up the diagram $D$ into its constituent pieces and assign a copy of the structure constants tensor $B \in \mathfrak{g}^{*} \otimes \mathfrak{g}^{*} \otimes \mathfrak{g}$ to each internal vertex $v$ of $D$ (keeping an association between the tensor factors in $\mathfrak{g}^{*} \otimes \mathfrak{g}^{*} \otimes \mathfrak{g}$ and the edges emanating from $v$, as dictated by the orientations of the edges and of the vertex $v$ itself). We assign the identity tensor in $\mathfrak{g}^{*} \otimes \mathfrak{g}$ to every arrow in $D$ that is not connected to an internal vertex, and contract any pair of factors connected by a fully internal arrow. The remaining tensor factors $\left(\mathfrak{g}^{*} \otimes \mathfrak{g}^{*} \otimes \mathfrak{g} \otimes \mathfrak{g} \otimes \mathfrak{g}^{*} \otimes \mathfrak{g}^{*}\right.$ in our examples) are all along the skeleton and can thus be ordered by the skeleton. We then multiply these factors to get an output $\mathcal{T}_{\mathfrak{g}}^{w}(D)$ in $\mathcal{U}(I \mathfrak{g})$.

It is also useful to restate this construction given a choice of a basis. Let $\left(x_{j}\right)$ be a basis of $\mathfrak{g}$ and let $\left(\varphi^{i}\right)$ be the dual basis of $\mathfrak{g}^{*}$, so that

$$
\varphi^{i}\left(x_{j}\right)=\delta_{j}^{i}
$$

and let $b_{i j}^{k}$ denote the structure constants of $\mathfrak{g}$ in the chosen basis:

$$
\left[x_{i}, x_{j}\right]=\sum b_{i j}^{k} x_{k}
$$

Mark every arrow in $D$ with lower case Latin letter from within ${ }^{31}\{i, j, k, \ldots\}$. Form a product $P_{D}$ by taking one $b_{\alpha \beta}^{\gamma}$ factor for each internal vertex $v$ of $D$ using the letters marking the edges around $v$ for $\alpha, \beta$ and $\gamma$ and by taking one $x_{\alpha}$ or $\varphi^{\beta}$ factor for each skeleton vertex of $D$, taken in the order that they appear along the long line skeleton, with the indices $\alpha$ and $\beta$ dictated by the edge markings and with the choice between factors in $\mathfrak{g}$ and factors in $\mathfrak{g}^{*}$ dictated by the orientations of the edges. Finally

${ }^{31}$ The supply of these can be made inexhaustible by the addition of numerical subscripts. 
let $\mathcal{T}_{\mathfrak{g}}^{w}(D)$ be the sum of $P_{D}$ over the indices $i, j, k, \ldots$ running from 1 to $\operatorname{dim} \mathfrak{g}$ :

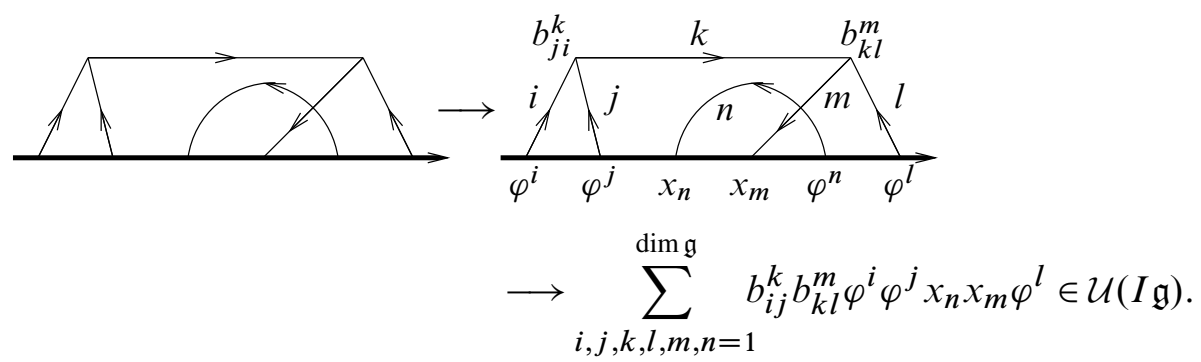

The next proposition is easy to verify (compare with [6, Theorem 4 and Exercise 5.1]).

Proposition 3.22 The above two definitions of $T_{\mathfrak{g}}^{w}$ agree, are independent of the choices made within them, and respect all the relations defining $\mathcal{A}^{w}$.

While we do not provide a proof of this proposition here, it is worthwhile stating the correspondence between the relations defining $\mathcal{A}^{w}$ and the Lie algebraic information in $\mathcal{U}(I \mathfrak{g})$ :

- $\overrightarrow{\mathrm{AS}}$ is the antisymmetry of the bracket of $\mathfrak{g}$.

- $\overrightarrow{\mathrm{IHX}}$ is the Jacobi identity of $\mathfrak{g}$.

- $\overrightarrow{\mathrm{STU}}_{1}$ and $\overrightarrow{\mathrm{STU}}_{2}$ are the relations $\left[x_{i}, x_{j}\right]=x_{i} x_{j}-x_{j} x_{i}$ and $\left[\varphi^{i}, x_{j}\right]=\varphi^{i} x_{j}-$ $x_{j} \varphi^{i}$ in $\mathcal{U}(I \mathfrak{g})$.

- TC is the fact that $\mathfrak{g}^{\star}$ is taken as an abelian algebra.

- $\overrightarrow{4 \mathrm{~T}}$ is the fact that the identity tensor in $\mathfrak{g}^{*} \otimes \mathfrak{g}$ is $\mathfrak{g}$-invariant.

3.5.3 Example: the 2-dimensional non-abelian Lie algebra Let $\mathfrak{g}$ be the Lie algebra with two generators $x_{1,2}$ satisfying $\left[x_{1}, x_{2}\right]=x_{2}$, so that the only non-vanishing structure constants $b_{i j}^{k}$ of $\mathfrak{g}$ are $b_{12}^{2}=-b_{21}^{2}=1$. Let $\varphi^{i} \in \mathfrak{g}^{*}$ be the dual basis of $x_{i}$; by an easy calculation, we find that in $I \mathfrak{g}$ the element $\varphi^{1}$ is central, while $\left[x_{1}, \varphi^{2}\right]=-\varphi^{2}$ and $\left[x_{2}, \varphi^{2}\right]=\varphi^{1}$. We calculate $\mathcal{T}_{\mathfrak{g}}^{w}\left(D_{L}\right), \mathcal{T}_{\mathfrak{g}}^{w}\left(D_{R}\right)$ and $\mathcal{T}_{\mathfrak{g}}^{w}\left(w_{k}\right)$ using the "in basis" technique of Equation (19). The outputs of these calculations lie in $\mathcal{U}(I \mathfrak{g})$; we display these results in a PBW basis in which the elements of $\mathfrak{g}^{*}$ precede the elements of $\mathfrak{g}$ :

$$
\begin{aligned}
& \mathcal{T}_{\mathfrak{g}}^{w}\left(D_{L}\right)=x_{1} \varphi^{1}+x_{2} \varphi^{2}=\varphi^{1} x_{1}+\varphi^{2} x_{2}+\left[x_{2}, \varphi^{2}\right]=\varphi^{1} x_{1}+\varphi^{2} x_{2}+\varphi_{1}, \\
& \mathcal{T}_{\mathfrak{g}}^{w}\left(D_{R}\right)=\varphi^{1} x_{1}+\varphi^{2} x_{2}, \\
& \mathcal{T}_{\mathfrak{g}}^{w}\left(w_{k}\right)=\left(\varphi^{1}\right)^{k}
\end{aligned}
$$


For the last assertion above, note that all non-vanishing structure constants $b_{i j}^{k}$ in our case have $k=2$, and therefore all indices corresponding to edges that exit an internal vertex must be set equal to 2 :

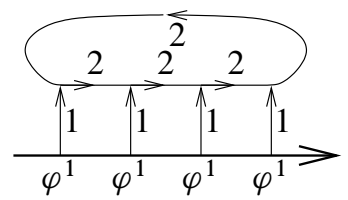

This forces the "hub" of $w_{k}$ to be marked 2 and therefore the legs to be marked 1 , and therefore $w_{k}$ is mapped to $\left(\varphi^{1}\right)^{k}$.

Note that the calculations in (20) are consistent with the relation $D_{L}-D_{R}=w_{1}$ of Theorem 3.15 and that they show that other than that relation, the generators of $\mathcal{A}^{w}$ are linearly independent.

\subsection{The Alexander polynomial}

Let $K$ be a long w-knot, and let $Z(K)$ be the invariant of Theorem 3.10. Theorem 3.26 below asserts that apart from self-linking, $Z(K)$ contains precisely the same information as the Alexander polynomial $A(K)$ of $K$ (recalled below). But we have to start with some definitions.
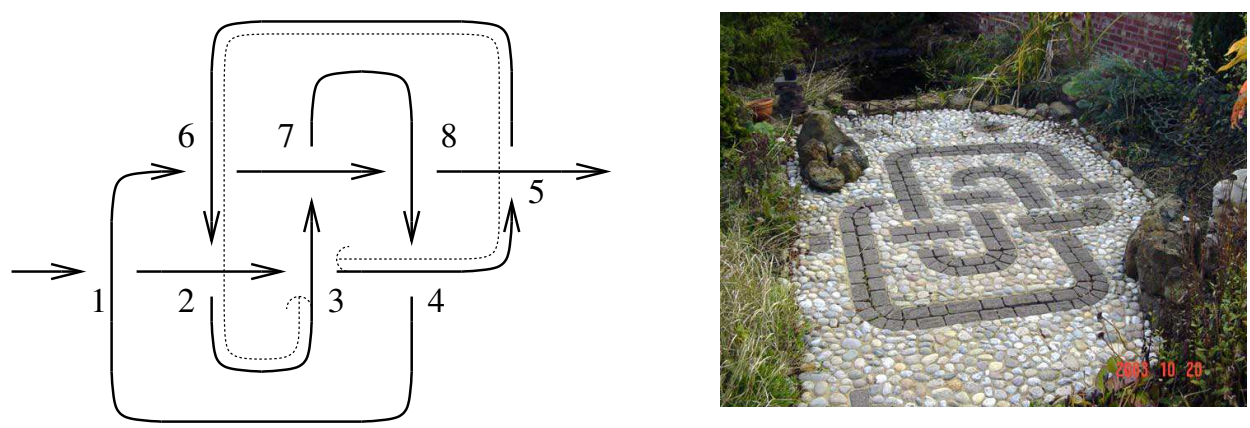

Figure 16: A long $8_{17}$, with the span of crossing \#3 marked. The projection is as in Brian Sanderson's garden. See [15]/SandersonsGarden.html.

Definition 3.23 Enumerate the crossings of $K$ from 1 to $n$ in some arbitrary order. For $1 \leq j \leq n$, the "span" of crossing $\# i$ is the connected open interval along the line parametrizing $K$ between the two times $K$ "visits" crossing \#i (see Figure 16). Form a matrix $T=T(K)$ with $T_{i j}$ the indicator function for whether "the lower strand of crossing \# $j$ is within the span of crossing \# $\#$ " (so $T_{i j}$ is 1 if for a given $i, j$ the quoted statement is true, and 0 otherwise). Let $s_{i}$ be the sign of crossing $\# i$ (recall 
that $>$ is positive, $\lambda$ is negative; so $(-,-,-,-,+,+,+,+)$ for Figure 16$)$, let $d_{i}$ be +1 if $K$ visits the "over" strand of crossing \# $i$ before visiting the "under" strand of that crossing, and let $d_{i}=-1$ otherwise (so $(-,+,-,+,-,+,-,+)$ for Figure 16). Let $S=S(K)$ be the diagonal matrix with $S_{i i}=s_{i} d_{i}$, and for an indeterminate $X$, let $X^{-S}$ denote the diagonal matrix with diagonal entries $X^{-s_{i} d_{i}}$. Finally, let $A(K)$ be the Laurent polynomial in $\mathbb{Z}\left[X, X^{-1}\right]$ given by

$$
A(K)(X):=\operatorname{det}\left(I+T\left(I-X^{-S}\right)\right) \text {. }
$$

Example 3.24 For the knot diagram in Figure 16,

$$
\begin{aligned}
T & =\left(\begin{array}{llllllll}
0 & 1 & 1 & 1 & 1 & 0 & 1 & 0 \\
0 & 0 & 1 & 0 & 1 & 0 & 0 & 0 \\
0 & 1 & 0 & 0 & 1 & 0 & 0 & 0 \\
0 & 1 & 0 & 0 & 1 & 0 & 1 & 0 \\
0 & 1 & 0 & 1 & 0 & 1 & 1 & 1 \\
0 & 1 & 0 & 1 & 0 & 0 & 1 & 0 \\
0 & 0 & 0 & 1 & 0 & 1 & 0 & 0 \\
0 & 0 & 0 & 1 & 0 & 1 & 0 & 0
\end{array}\right), \quad S=\left(\begin{array}{cccccccc}
1 & 0 & 0 & 0 & 0 & 0 & 0 & 0 \\
0 & -1 & 0 & 0 & 0 & 0 & 0 & 0 \\
0 & 0 & 1 & 0 & 0 & 0 & 0 & 0 \\
0 & 0 & 0 & -1 & 0 & 0 & 0 & 0 \\
0 & 0 & 0 & 0 & -1 & 0 & 0 & 0 \\
0 & 0 & 0 & 0 & 0 & 1 & 0 & 0 \\
0 & 0 & 0 & 0 & 0 & 0 & -1 & 0 \\
0 & 0 & 0 & 0 & 0 & 0 & 0 & 1
\end{array}\right), \\
A & =\left(\begin{array}{cccccccccc}
1 & 1-X & 1-X^{-1} & 1-X & 1-X & 0 & 1-X & 0 \\
0 & 1 & 1-X^{-1} & 0 & 1-X & 0 & 0 & 0 \\
0 & 1-X & 1 & 0 & 1-X & 0 & 0 & 0 \\
0 & 1-X & 0 & 1 & 1-X & 0 & 1-X & 0 \\
0 & 1-X & 0 & 1-X & 1-X & 1-X^{-1} & 1-X & 1-X^{-1} \\
0 & 1-X & 0 & 1-X & 0 & 1 & 1-X & 0 \\
0 & 0 & 0 & 1-X & 0 & 1-X^{-1} & 1 & 0 \\
0 & 0 & 0 & 1-X & 0 & 1-X^{-1} & 0 & 1
\end{array} \mid .\right.
\end{aligned}
$$

The last determinant equals $-X^{3}+4 X^{2}-8 X+11-8 X^{-1}+4 X^{-2}-X^{-3}$, the Alexander polynomial of the knot $8_{17}$ (see eg [79]).

Theorem 3.25 (Lee, [67, Theorem 1]) For any (classical) knot $K, A(K)$ is equal to the normalized Alexander polynomial [79] of $K$.

The Mathematica notebook [15, "wA"] verifies Theorem 3.25 for all prime knots with up to 11 crossings.

The following theorem says that $Z(K)$ can be computed from $A(K)$ (see Equation (22)) and that modulo a certain additional relation and with the appropriate identifications in place, $Z(K)$ is $A(K)$ (see Equation (23)). 
Theorem 3.26 (Proof in Section 3.7) Let $x$ be an indeterminate, let sl be selflinking as in Exercise 3.6, let $D_{A}:=D_{L}=D_{R}$ and $w_{k}$ be as in Figure 14, and let $w: \mathbb{Q} \llbracket x \rrbracket \rightarrow \mathcal{A}^{w}$ be the linear map defined by $x^{k} \mapsto w_{k}$. Then for a long $w$-knot $K$,

$$
Z(K)=\underbrace{\exp _{\mathcal{A}^{s w}}\left(\operatorname{sl}(K) D_{A}\right)}_{\text {sl coded in arrows }} \cdot \underbrace{\exp _{\mathcal{A}^{s w}}\left(-w\left(\log _{\mathbb{Q} \llbracket x \rrbracket} A(K)\left(e^{x}\right)\right)\right),}_{\text {main part: Alexander coded in wheels }}
$$

where the logarithm and inner exponentiation are computed by formal power series in $\mathbb{Q} \llbracket x \rrbracket$ and the outer exponentiations are likewise computed in $\mathcal{A}^{\text {sw }}$.

Let $\mathcal{A}^{\text {reduced }}$ be $\mathcal{A}^{s w}$ modulo the additional relations $D_{A}=0$ and $w_{k} w_{l}=w_{k+l}$ for $k, l \neq 1$ :

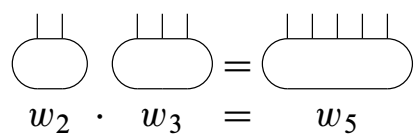

The quotient $\mathcal{A}^{\text {reduced }}$ can be identified with the vector space of (infinite) linear combinations of the $w_{k}$, with $k \neq 1$. Identifying the $k$-wheel $w_{k}$ with $x^{k}$, we see that $\mathcal{A}^{\text {reduced }}$ is the space of power series in $x$ having no linear terms. Note by inspecting Equation (21) that $A(K)\left(e^{x}\right)$ never has a term linear in $x$, and that modulo $w_{k} w_{l}=w_{k+l}$, the exponential and the logarithm in Equation (22) cancel each other out. Hence, within $\mathcal{A}^{\text {reduced }}$

$$
Z(K)=A^{-1}(K)\left(e^{x}\right) .
$$

Remark 3.27 In [47] Habiro, Kanenobu, and Shima show that all coefficients of the Alexander polynomial are finite-type invariants of w-knots, and in [48] Habiro and Shima show that all finite-type invariants of w-knots are polynomials in the coefficients of the Alexander polynomial. Thus, Theorem 3.26 is merely an "explicit form" of these earlier results.

\subsection{Proof of Theorem 3.26}

We start with a sketch. The proof of Theorem 3.26 can be divided into three parts: differentiation, bulk management, and computation.

Differentiation Both sides of our goal, that is, Equation (22), are exponential in nature. When seeking to show an equality of exponentials it is often beneficial to compare their derivatives. ${ }^{32}$ In our case the useful "derivatives" to use are the "Euler operator" $E$ ("multiply every term by its degree", an analogue of $f \mapsto x f^{\prime}$, defined

${ }^{32}$ Thanks, Dylan. 
in Section 3.7.1), and the "normalized Euler operator" $Z \mapsto \widetilde{E} Z:=Z^{-1} E Z$, which is a variant of the logarithmic derivative $f \mapsto x(\log f)^{\prime}=x f^{\prime} / f$. Since $\widetilde{E}$ is one to one (see Section 3.7.1) and since we know how to apply $\widetilde{E}$ to the right hand side of Equation (22) (see Section 3.7.1), it is enough to show that with $B:=T(\exp (-x S)-I)$ and suppressing the fixed w-knot $K$ from the notation,

$$
E Z=Z \cdot\left(s l \cdot D_{A}-w\left[x \operatorname{tr}\left((I-B)^{-1} T S \exp (-x S)\right)\right]\right) \quad \text { in } \mathcal{A}^{s w} .
$$

Bulk management Next we seek to understand the left-hand side of Equation (24). $Z$ is made up of "quantities in bulk": arrows that come in exponential "reservoirs". As it turns out, $E Z$ is made up of the same bulk quantities, but also allowing for a single non-bulk "excitation", which we often highlight in red. (Compare with $E e^{x}=x \cdot e^{x}$; the "bulk" $e^{x}$ remains, and single "excited red" $x$ gets created). We wish to manipulate and simplify that red excitation. This is best done by introducing a certain module, $I A M_{K}$, the "infinitesimal Alexander module" of $K$ (see Section 3.7.2). The elements of $I A M_{K}$ can be thought of as names for "bulk objects with a red excitation", and hence there is an "interpretation map" $\iota: I A M_{K} \rightarrow \mathcal{A}^{s w}$, which maps every "name" into the object it represents. There are three special elements in $I A M_{K}$ : an element $\lambda$, which is the name of $E Z$ (that is, $\iota(\lambda)=E Z$ ), the element $\delta_{A}$ which is the name of $D_{A} \cdot Z$ (so $\iota\left(\delta_{A}\right)=D_{A} \cdot Z$ ), and an element $\omega_{1}$ which is the name of a "detached" $1-$ wheel that is appended to $Z$. The latter can take a coefficient which is a power of $x$, with $\iota\left(x^{k} \omega_{1}\right)=w\left(x^{k+1}\right) \cdot Z=(Z$ times a $(k+1)$-wheel $)$. Thus, it is enough to show that in $I A M_{K}$,

$$
\lambda=s l \cdot \delta_{A}-\operatorname{tr}\left((I-B)^{-1} T S X^{-S}\right) \omega_{1}, \quad \text { with } X=e^{x} .
$$

Indeed, applying $\iota$ to both sides of the above equation, we get Equation (24) back again.

Computation Last, we show in Section 3.7.3 that Equation (25) holds true. This is a computation that happens entirely in $I A M_{K}$ and does not mention finite-type invariants, expansions or arrow diagrams in any way.

3.7.1 The Euler operator Let $A$ be a completed, graded algebra with unit, in which all degrees are $\geq 0$. Define a continuous linear operator $E: A \rightarrow A$ by setting $E a=$ $(\operatorname{deg} a) a$ for homogeneous $a \in A$. In the case $A=\mathbb{Q} \llbracket x \rrbracket$, we have $E f=x f^{\prime}$, the standard "Euler operator"; indeed, for each $n, E x^{n}=n x^{n}=x \cdot\left(x^{n}\right)^{\prime}$. Hence, we adopt the name $E$ for this operator in general.

We say that $Z \in A$ is a "perturbation of the identity" if its degree 0 piece is 1 . Such a $Z$ is always invertible. For such a $Z$, set $\widetilde{E} Z:=Z^{-1} \cdot E Z$, and call the thus (partially) defined operator $\widetilde{E}: A \rightarrow A$ the "normalized Euler operator". From this point on when 
we write $\widetilde{E} Z$ for some $Z \in A$, we automatically assume that $Z$ is a perturbation of the identity or that it is trivial to show that $Z$ is a perturbation of the identity. Note that for $f \in \mathbb{Q} \llbracket x \rrbracket$, we have $\widetilde{E} f=x(\log f)^{\prime}$, so $\widetilde{E}$ is a variant of the logarithmic derivative.

Claim 3.28 $\widetilde{E}$ is one to one.

Proof Assume $Z_{1} \neq Z_{2}$ and let $d$ be the smallest degree in which they differ. Then $d>0$ and in degree $d$ the difference $\widetilde{E} Z_{1}-\widetilde{E} Z_{2}$ is $d$ times the difference $Z_{1}-Z_{2}$, and hence, $\widetilde{E} Z_{1} \neq \widetilde{E} Z_{2}$.

Thus, in order to prove our goal — that is, Equation (22) — it is enough to compute $\widetilde{E}$ of both sides and to show the equality then. We start with the right-hand side of Equation (22); but first, we need some simple properties of $E$ and $\widetilde{E}$. The proofs of these properties are routine and hence are omitted.

Proposition 3.29 The following hold true:

(1) $E$ is a derivation: $E(f g)=(E f) g+f(E g)$.

(2) If $Z_{1}$ commutes with $Z_{2}$, then $\widetilde{E}\left(Z_{1} Z_{2}\right)=\widetilde{E} Z_{1}+\widetilde{E} Z_{2}$.

(3) If $z$ commutes with $E z$, then $E e^{z}=e^{z}(E z)$ and $\widetilde{E} e^{z}=E z$.

(4) If $w: A \rightarrow \mathcal{A}$ is a morphism of graded algebras, then it commutes with $E$ and $\tilde{E}$.

Let us denote the right-hand side of Equation (22) by $Z_{1}(K)$. Then, by the above proposition, remembering (see Theorem 3.15) that $\mathcal{A}^{s w}$ is commutative and that $\operatorname{deg} D_{A}=1$, we have

$$
\widetilde{E} Z_{1}(K)=s l \cdot D_{A}-w\left(E \log A(K)\left(e^{x}\right)\right)=s l \cdot D_{A}-w\left(x \frac{d}{d x} \log A(K)\left(e^{x}\right)\right) .
$$

The rest is an exercise in matrices and differentiation. $A(K)$ is a determinant, see Equation (21), and in general $\frac{d}{d x} \log \operatorname{det}(M)=\operatorname{tr}\left(M^{-1} \frac{d}{d x} M\right)$. So with $B=T\left(e^{-x S_{-}}\right.$ $I)$, and so $M=I-B$, we have

$$
\begin{aligned}
\widetilde{E} Z_{1}(K) & =s l \cdot D_{A}+w\left(x \operatorname{tr}\left((I-B)^{-1} \frac{d}{d x} B\right)\right) \\
& =s l \cdot D_{A}-w\left(x \operatorname{tr}\left((I-B)^{-1} T S e^{-x S}\right)\right),
\end{aligned}
$$

as promised in Equation (24). 
3.7.2 The infinitesimal Alexander module Let $K$ be a w-knot diagram. The "infinitesimal Alexander module" $I A M_{K}$ of $K$, which is defined in detail below, is a certain module made from a certain space $I A M_{K}^{0}$ of pictures "annotating" $K$ with "red excitations" modulo some pictorial relations that indicate how the red excitations can be moved around. The space $I A M_{K}^{0}$ in itself is made of three pieces, or "sectors": the "A sector" in which the excitations are red arrows, the "Y sector" in which the excitations are "red hairy Y-diagrams", and a rank 1 "W sector" for "red hairy wheels". There is an "interpretation map" $\iota: I A M_{K}^{0} \rightarrow \mathcal{A}^{w}$ which descends to a well-defined (and homonymous) $\iota: I A M_{K} \rightarrow \mathcal{A}^{w}$. Finally, there are some special elements $\lambda$ and $\delta_{A}$ that live in the A sector of $I A M_{K}^{0}$, and $\omega_{1}$ that lives in the $\mathrm{W}$ sector.

In principle, the description of $I A M_{K}^{0}$ and of $I A M_{K}$ can be given independently of the interpretation map $\iota$, and there are some good questions to ask about $I A M_{K}$ (and the special elements in it) that are completely independent of the interpretation of the elements of $I A M_{K}$ as "perturbed bulk quantities" within $\mathcal{A}^{s w}$. Yet $I A M_{K}$ is a complicated object and we fear its definition will appear completely artificial without its interpretation. Hence, below the two definitions will be woven together.

$I A M_{K}$ and $\iota$ may equally well be described in terms of $K$ or in terms of the Gauss diagram of $K$ (see Remark 3.4). For pictorial simplicity, we choose to use the latter; so let $G=G(K)$ be the Gauss diagram of $K$. It is best to read the following definition while at the same time studying Figure 17.

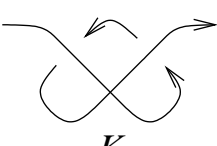

$K$
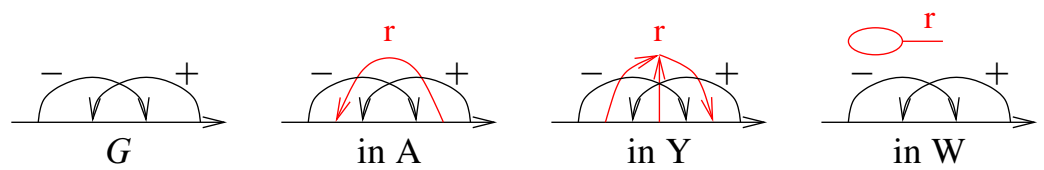

Figure 17: A sample w-knot $K$, its Gauss diagram $G$, and one generator from each of the A, Y, and W sectors of $I A M_{K}^{0}$. Red parts are marked with the letter $\mathrm{r}$.

Definition 3.30 Let $R$ be the ring $\mathbb{Z}\left[X, X^{-1}\right]$ of Laurent polynomials in a variable $X$ with integer coefficients, ${ }^{33}$ and let $R_{1}$ be the subring of polynomials that vanish at $X=1$ (ie whose sum of coefficients is 0 ). ${ }^{34}$ Let $I A M_{K}^{0}$ be the direct sum of the following three modules (which for the purpose of taking the direct sum are all regarded as $\mathbb{Z}$-modules):

${ }^{33}$ Later, $X$ is interpreted in $\mathcal{A}^{w}$ as a formal exponential $e^{x}$. So within IAM we can restrict to coefficients in $\mathbb{Z}$, yet in $\mathcal{A}^{w}$ we must allow coefficients in $\mathbb{Q}$.

${ }^{34} R_{1}$ is only very lightly needed, and only within Definition 3.31. In particular, all that we say about $I A M_{K}$ that does not concern the interpretation map $\iota$ is equally valid with $R$ replacing $R_{1}$. 
(1) The "A sector" is the free $\mathbb{Z}$-module generated by all diagrams made from $G$ by the addition of a single unmarked "red excitation" arrow, whose endpoints are on the long line skeleton of $G$ and are distinct from each other and from all other endpoints of arrows in $G$. Such diagrams are considered combinatorially: so two are equivalent if and only if they differ only by an orientation-preserving diffeomorphism of the skeleton. Let us count: if $K$ has $n$ crossings, then $G$ has $n$ arrows and the skeleton of $G$ gets subdivided into $m:=2 n+1$ arcs. An A sector diagram is specified by the choice of an arc for the tail of the red arrow and an arc for the head ( $m^{2}$ choices), except if the head and the tail fall within the same arc, then their relative ordering has to be specified as well ( $m$ further choices). So the rank of the A sector over $\mathbb{Z}$ is $m(m+1)$.

(2) The "Y sector" is the free $R_{1}$-module generated by all diagrams made from $G$ by the addition of a single "red excitation" $Y$-shape single-vertex graph, with two incoming edges ("tails") and one outgoing ("head"), modulo anti-symmetry for the two incoming edges (again, considered combinatorially). Counting is more elaborate: when the three edges of the $Y$ end in distinct arcs in the skeleton of $G$, we have $\frac{1}{2} m(m-1)(m-2)$ possibilities ( $\frac{1}{2}$ for the antisymmetry). When the two tails of the $Y$ lie on the same arc, we get 0 by anti-symmetry. The remaining possibility is to have the head and one tail on one arc (order matters!) and the other tail on another, at $2 m(m-1)$ possibilities. So the rank of the Y sector over $R_{1}$ is $m(m-1)\left(\frac{1}{2} m+1\right)$.

(3) The "W sector" is the rank 1 free $R$-module with a single generator $w_{1}$. It is not necessary for $w_{1}$ to have a pictorial representation, yet one, involving a single "red" 1-wheel, is shown in Figure 17. This pictorial representation is consistent with the interpretation in the definition below of $\omega_{1}$ as a detached $1-$ wheel.

Definition 3.31 The "interpretation map" $\iota: I A M_{K}^{0} \rightarrow \mathcal{A}^{w}$ is defined by sending the arrows (marked + or - ) of a diagram in $I A M_{K}^{0}$ to $\left(e^{ \pm a}\right)$-exponential reservoirs of arrows, as in the definition of $Z$ (see Remark 3.11). In addition, the red excitations of diagrams in $I A M_{K}^{0}$ are interpreted as follows:

(1) In the A sector, the red arrow is simply mapped to itself, with the colour red suppressed.

(2) In the $\mathrm{Y}$ sector, diagrams have red $Y$ s and coefficients $f \in R_{1}$. Substitute $X=e^{x}$ in $f$, expand in powers of $x$, and interpret $x^{k} Y$ as a "hairy $Y$ with $k-1$ hairs" as in Exercise 3.20. Note that $f(1)=0$, so only positive powers of $x$ occur. So we never need to worry about " $Y$ s with -1 hairs". This is the only point where the condition $f \in R_{1}$ (as opposed to $f \in R$ ) is needed. 
(3) In the $\mathrm{W}$ sector treat the coefficients as above, but interpret $x^{k} w_{1}$ as a detached $w_{k+1}$, ie as a detached wheel with $k+1$ spokes, as in Exercise 3.20.

As stated above, $I A M_{K}$ is the quotient of $I A M_{K}^{0}$ by some set of relations. The best way to think of this set of relations is as "everything that's obviously annihilated by $\iota$ ". Here's the same thing, in a more formal language.

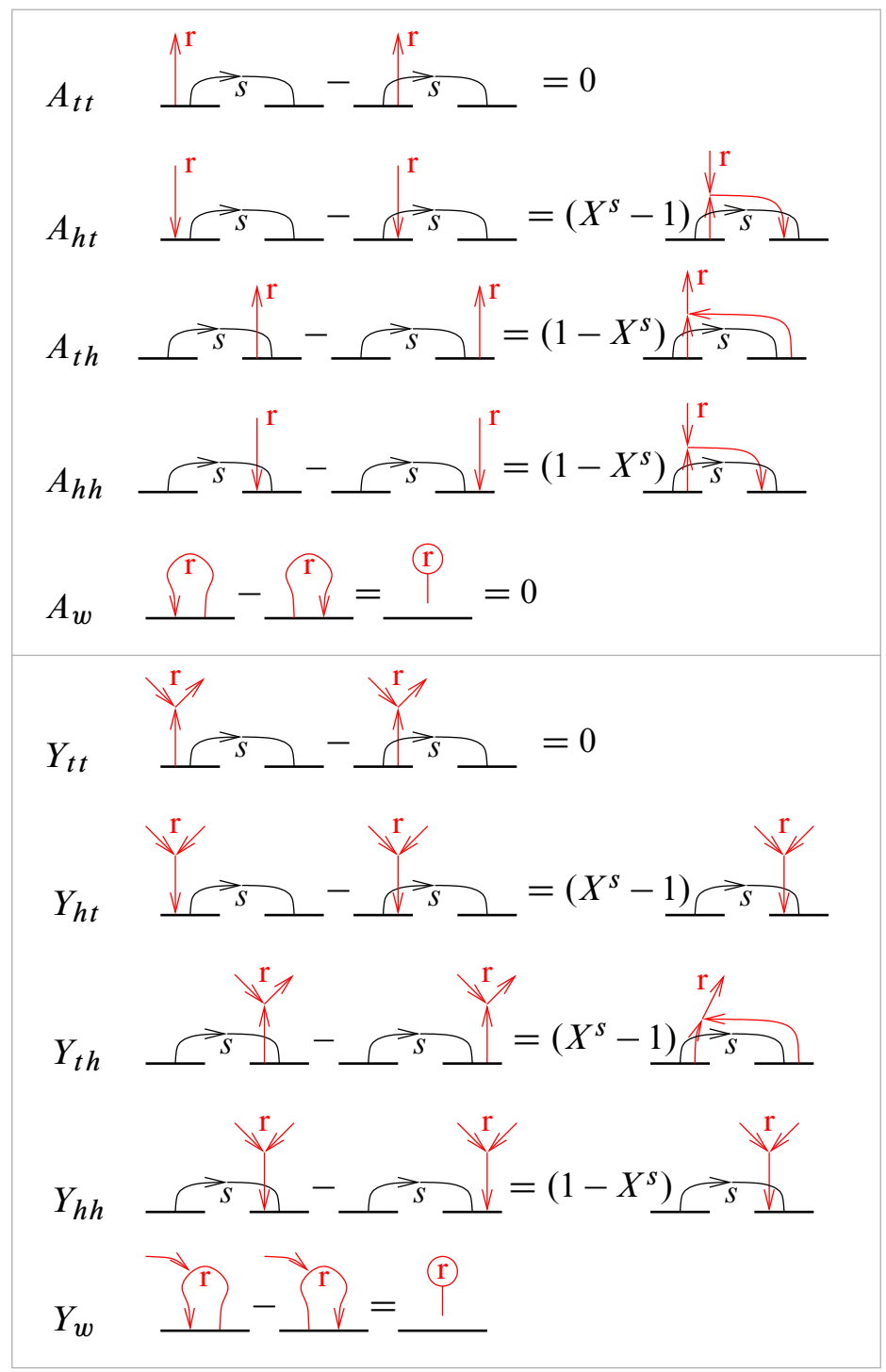

Figure 18: The relations $\mathcal{R}$ making $I A M_{K}$. 
Definition 3.32 Let $I A M_{K}:=I A M_{K}^{0} / \mathcal{R}$, where $\mathcal{R}$ is the linear span of the relations depicted in Figure 18. The top 8 relations are about moving a leg of the red excitation across an arrow head or an arrow tail in $G$. Since the red excitation may be either an arrow $A$ or a $Y$, its leg in motion may be either a tail or a head, and it may be moving either past a tail or past a head, there are 8 relations of that type. The $A_{w}$ relation corresponds to $D_{L}-D_{R}=w_{1}=0$. The $Y_{w}$ relation indicates the "price" (always a red $w_{1}$ ) of commuting a red head across a red tail. As per custom, in each case only the changing part of the diagrams involved is shown. Further, the red excitations are marked with the letter " $r$ " and the sign of an arrow in $G$ is marked $s$; so we always have $s \in\{ \pm 1\}$. The relations in the left column may be multiplied by a scalar in $\mathbb{Z}$, while the relations in the right column may be multiplied by a scalar in $R$. Hence, for example, $x^{0} w_{1}=0$ by $A_{w}$, yet $x^{k} w_{1} \neq 0$ for $k>0$.

Proposition 3.33 The interpretation map $\iota$ indeed annihilates all the relations in $\mathcal{R}$.

Proof Both $\iota A_{t t}$ and $\iota Y_{t t}$ follow immediately from the TC relation. The formal identity $e^{\text {ad } b}(a)=e^{b} a e^{-b}$ (here ad denotes the adjoint representation) implies $e^{\operatorname{ad} b}(a) e^{b}=e^{b} a$, and hence, $a e^{b}-e^{b} a=\left(1-e^{\mathrm{ad} b}\right)(a) e^{b}$. With $a$ interpreted as "red head", $b$ as "black head", and ad $b$ as "hair" (justified by the $\iota$-meaning of hair and by the $\overrightarrow{\mathrm{STU}}_{1}$ relation, see Figure 12$)$, the last equality becomes a proof of $\iota Y_{h h}$. Further pushing that same equality, we get

$$
a e^{b}-e^{b} a=\frac{1-e^{\operatorname{ad} b}}{\operatorname{ad} b}([b, a]),
$$

where $\left(1-e^{\mathrm{ad} b}\right) / \mathrm{ad} b$ is first interpreted as a power series $\left(1-e^{y}\right) / y$ involving only non-negative powers of $y$, and then the substitution $y=\operatorname{ad} b$ is made. But that's $\iota A_{h h}$, when one remembers that $\iota$ on the $Y$ sector automatically contains a single " $1 /$ hair" factor. Similar arguments, though using $\overrightarrow{\mathrm{STU}}_{2}$ instead of $\overrightarrow{\mathrm{STU}}_{1}$, prove that $Y_{h t}, Y_{t h}$, $A_{h t}$, and $A_{t h}$ are all in ker $\iota$. Finally, $\iota A_{w}$ is RI, and $\iota Y_{w}$ is a direct consequence of $\overrightarrow{\mathrm{STU}}_{2}$.

Finally, we come to the special elements $\lambda, \delta_{A}$, and $\omega_{1}$.

Definition 3.34 Within $I A M_{G}$, let $\omega_{1}$ be, as before, the generator of the $\mathrm{W}$ sector. Let $\delta_{A}$ be a "short" red arrow, as in the $A_{w}$ relation (exercise: modulo $\mathcal{R}$, this is independent of the placement of the short arrows within $G$ ). Finally, let $\lambda$ be the signed sum of exciting each of the (black) arrows in $G$ in turn. The picture says all, and it is Figure 19. 


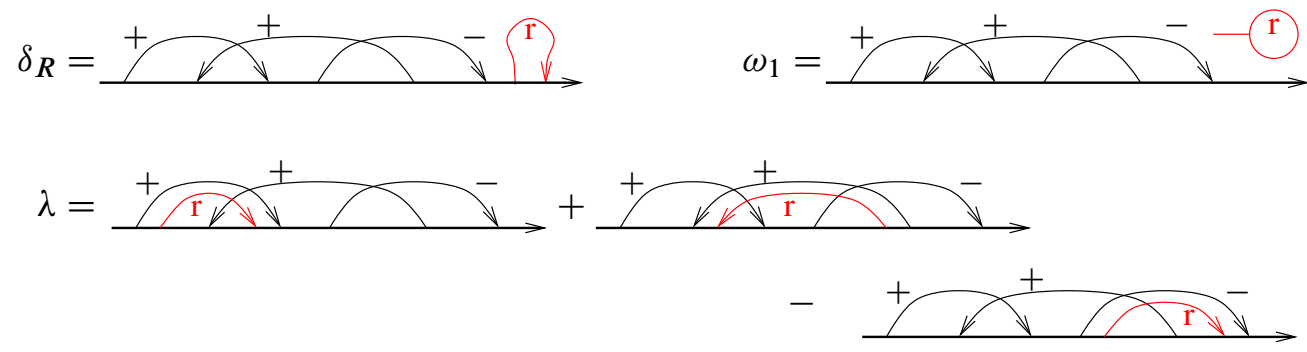

Figure 19: The special elements $\omega_{1}, \delta_{A}$, and $\lambda$ in $I A M_{G}$, for a sample 3-arrow Gauss diagram $G$.

Lemma 3.35 In $\mathcal{A}^{s w}(\uparrow)$, the special elements of IAM $_{G}$ are interpreted as follows: $\iota\left(\omega_{1}\right)=Z w_{1}, \iota\left(\delta_{A}\right)=Z D_{A}$, and most interestingly, $\iota(\lambda)=E Z$. Thus Equation (25) (if true) implies Equation (24) and hence, it implies our goal, Theorem 3.26.

Proof For the proof of this lemma, the only thing that isn't done yet and isn't trivial is the assertion $\iota(\lambda)=E Z$. But this assertion is a consequence of $E e^{ \pm a}= \pm a e^{ \pm a}$ and of a Leibniz law for the derivation $E$, appropriately generalized to a context where $Z$ can be thought of as a "product" of "arrow reservoirs". The details are left to the reader.

3.7.3 The computation of $\lambda$ Naturally, our next task is to prove Equation (25). This is done entirely algebraically within the finite rank module $I A M_{G}$. To read this section one need not know about $\mathcal{A}^{s w}(\uparrow)$, or $\iota$, or $Z$, but we do need to lay out some notation. Start by marking the arrows of $G$ with $a_{1}$ through $a_{n}$ in some order.

Let $\epsilon$ stand for the informal yet useful quantity "a little". Let $\lambda_{i j}$ denote the difference $\lambda_{i j}^{\prime}-\lambda_{i j}^{\prime \prime}$ of red excitations in the A sector of $I A M_{G}$, where $\lambda_{i j}^{\prime}$ is the diagram with a red arrow whose tail is $\epsilon$ to the right of the left end of $a_{i}$ and whose head is $\frac{1}{2} \epsilon$ away from the head of $a_{j}$ in the direction of the tail of $a_{j}$, and where $\lambda_{i j}^{\prime \prime}$ has a red arrow whose tail is $\epsilon$ to the left of the right end of $a_{i}$ and whose head is as before, $\frac{1}{2} \epsilon$ away from head of $a_{j}$ in the direction of the tail of $a_{j}$. Let $\Lambda=\left(\lambda_{i j}\right)$ be the matrix whose entries are the $\lambda_{i j}$, as shown in Figure 20.

Similarly, let $y_{i j}$ denote the element in the Y sector of $I A M_{G}$ whose red Y has its head $\frac{1}{2} \epsilon$ away from head of $a_{j}$ in the direction of the tail of $a_{j}$, its right tail (as seen from the head) $\epsilon$ to the left of the right end of $a_{i}$ and its left tail $\epsilon$ to the right of the left end of $a_{i}$. Let $Y=\left(y_{i j}\right)$ be the matrix whose entries are the $y_{i j}$, as shown in Figure 20.

Lemma 3.36 With $S$ and $T$ as in Definition 3.23, and with $B=T\left(X^{-S}-I\right)$ and $\lambda$ as above, the following identities between elements of IAM $_{G}$ and matrices with entries 

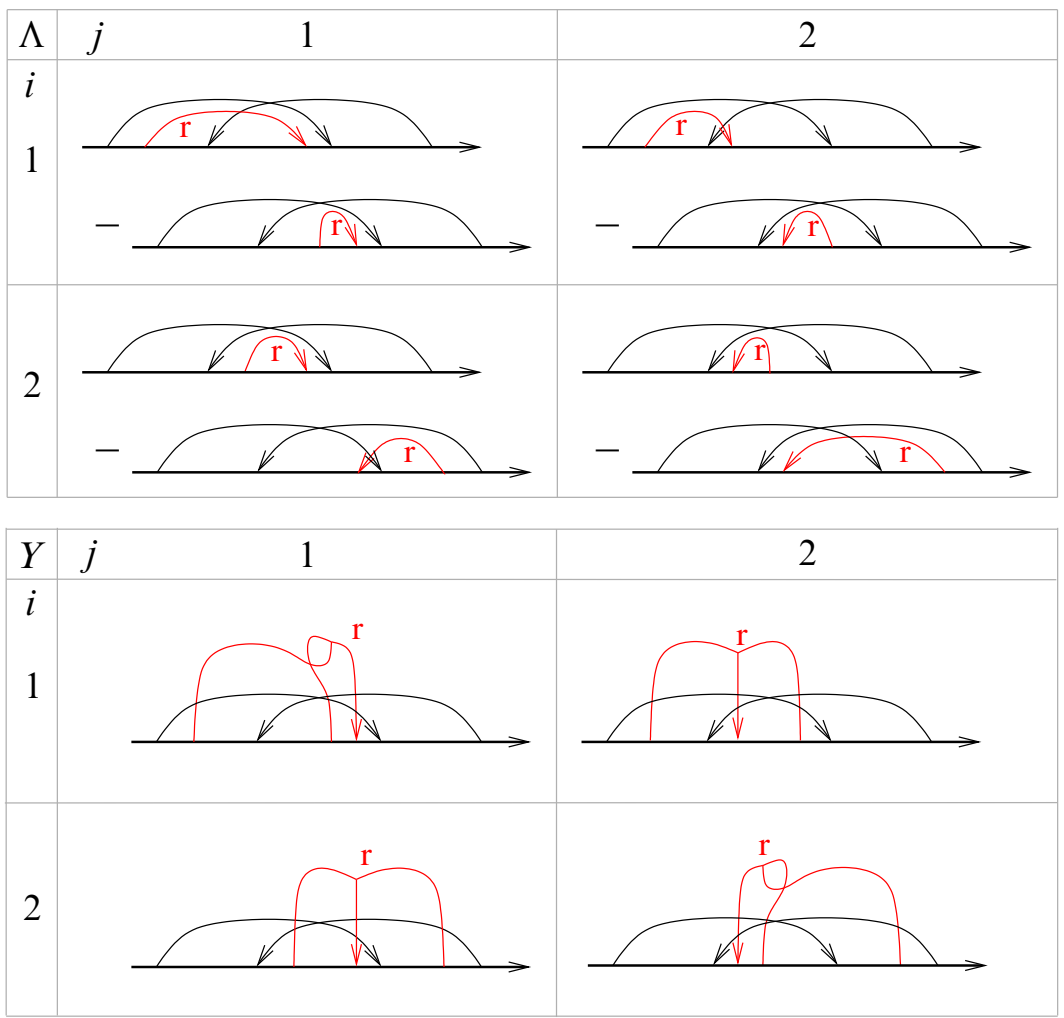

Figure 20: The matrices $\Lambda$ and $Y$ for a sample 2-arrow Gauss diagram (the signs on $a_{1}$ and $a_{2}$ are suppressed, and so are the $r$ marks). The twists in $y_{11}$ and $y_{22}$ may be replaced by minus signs.

in $\operatorname{IAM}_{G}$ hold true:

$$
\lambda-s l \cdot D_{A}=\operatorname{tr} S \Lambda,
$$

$$
\begin{aligned}
& \Lambda=-B Y-T X^{-S} w_{1}, \\
& Y=B Y+T X^{-S} w_{1} .
\end{aligned}
$$

Proof of Equation (25) given Lemma 3.36 The last of the equalities above implies that $Y=(I-B)^{-1} T X^{-S} w_{1}$. Thus,

$$
\begin{aligned}
\lambda-s l \cdot D_{A}=\operatorname{tr} S \Lambda & =-\operatorname{tr} S\left(B Y+T X^{-S} w_{1}\right) \\
& =-\operatorname{tr} S\left(B(I-B)^{-1} T X^{-S}+T X^{-S}\right) w_{1} \\
& =-\operatorname{tr}\left((I-B)^{-1} T S X^{-S}\right) w_{1},
\end{aligned}
$$

and this is exactly Equation (25). 
Proof of Lemma 3.36 Equation (26) is trivial. The proofs of Equations (27) and (28) both have the same simple cores, that have to be supplemented by highly unpleasant tracking of signs and conventions and powers of $X$. Let us start from the cores.

To prove Equation (27) we wish to "compute" $\lambda_{i k}=\lambda_{i k}^{\prime}-\lambda_{i k}^{\prime \prime}$. As $\lambda_{i k}^{\prime}$ and $\lambda_{i k}^{\prime \prime}$ have their heads in the same place, we can compute their difference by gradually sliding the tail of $\lambda_{i k}^{\prime}$ from its original position near the left end of $a_{i}$ towards the right end of $a_{i}$, where it would be cancelled by $\lambda_{i k}^{\prime \prime}$. As the tail slides we pick up a $y_{j k}$ term each time it crosses a head of an $a_{j}$ (relation $A_{t h}$ ), we pick up a vanishing term each time it crosses a tail (relation $A_{t t}$ ), and we pick up a $w_{1}$ term if the tail needs to cross over its own head (relation $\left.A_{w}\right)$. Ignoring signs and $\left(X^{ \pm 1}-1\right)$ factors, the sum of the $y_{j k}$-terms should be proportional to $T Y$, for indeed, the matrix $T$ has non-zero entries precisely when the head of an $a_{j}$ falls within the span of an $a_{i}$. Un-ignoring these signs and factors, we get $-B Y$ (recall that $B=T\left(X^{-S}-I\right)$ is just $T$ with added $\left(X^{ \pm 1}-1\right)$ factors). Similarly, a $w_{1}$ term arises in this process when a tail has to cross over its own head, that is, when the head of $a_{k}$ is within the span of $a_{i}$. Thus, the $w_{1}$ term should be proportional to $T w_{1}$, and we claim it is $-T X^{-S} w_{1}$.

The core of the proof of Equation (28) is more or less the same. We wish to "compute" $y_{i k}$ by sliding its left leg, starting near the left end of $a_{i}$, towards its right leg, which is stationary near the right end of $a_{i}$. When the two legs come together, we get 0 because of the anti-symmetry of $Y$ excitations. Along the way we pick up further $Y$ terms from the $Y_{t h}$ relations, and sometimes a $w_{1}$ term from the $Y_{w}$ relation. When all signs and $\left(X^{ \pm 1}-1\right)$ factors are accounted for, we get Equation (28).

We leave it to the reader to complete the details in the above proofs. It is a major headache, and we would not have trusted ourselves had we not written a computer program to manipulate quantities in $I A M_{G}$ by a brute force application of the relations in $\mathcal{R}$. Everything checks; see [15, "The Infinitesimal Alexander Module"].

This concludes the proof of Theorem 3.26.

Remark 3.37 We chose the name "infinitesimal Alexander module" as in our mind there is some similarity between $I A M_{K}$ and the "Alexander module" of $K$. Yet beyond the above, we did not embark on any serious study of $I A M_{K}$. In particular, we do not know if $I A M_{K}$ in itself is an invariant of $K$ (though we suspect it wouldn't be hard to show that it is), we do not know if $I A M_{K}$ contains any further information beyond $s l$ and the Alexander polynomial, and we do not know if there is any formal relationship between $I A M_{K}$ and the Alexander module of $K$. 
Remark 3.38 The logarithmic derivative of the Alexander polynomial also appears in Lescop's work, see $[69 ; 68]$. We don't know if its appearances there are related to its appearance here.

\subsection{The relationship with $u-k n o t s$}

Unlike in the case of braids, there is a canonical universal finite-type invariant of u-knots: the Kontsevich integral $Z^{u}$. So it makes sense to ask how it is related to the expansion $Z^{w}$.

We claim that the square

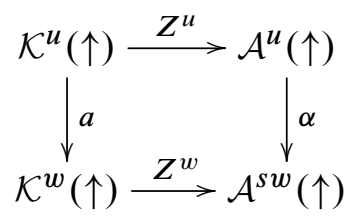

commutes, where $\mathcal{K}^{u}(\uparrow)$ stands for long u-knots (knottings of an oriented line), and similarly $\mathcal{K}^{w}(\uparrow)$ denotes long w-knots. As before, $a$ is the composition of the maps $\mathrm{u}$-knots $\rightarrow$ v-knots $\rightarrow$ w-knots, and $\alpha$ is the induced map on the associated graded spaces, mapping each chord to the sum of the two ways to direct it.

Recall that $\alpha$ kills everything but wheels and arrows (hence $Z^{w}$ is much weaker, but also easier to handle, than the Kontsevich integral). We are going to use the formula for the "wheel part" of the Kontsevich integral as stated in [58]. Let $K$ be a $0-$ framed long knot, and let $A(K)$ denote the Alexander polynomial. Then by [58],

$$
Z^{u}(K)=\exp _{\mathcal{A}^{u}}\left(-\left.\frac{1}{2} \log A(K)\left(e^{h}\right)\right|_{h^{2 n} \rightarrow w_{2 n}^{u}}\right)+\text { "loopy terms", }
$$

where $w_{2 n}^{u}$ stands for the unoriented wheel with $2 n$ spokes, and "loopy terms" means terms that contain diagrams with more than one loop, which are killed by $\alpha$. Note that by the symmetry $A(z)=A\left(z^{-1}\right)$ of the Alexander polynomial, $A(K)\left(e^{h}\right)$ contains only even powers of $h$, as suggested by the formula.

We need to understand how $\alpha$ acts on wheels. Due to the two-in-one-out rule, a wheel is zero unless all the "spokes" are oriented inward, and the cycle oriented in one direction. In other words, there are two ways to orient an unoriented wheel: clockwise or counterclockwise. Due to the anti-symmetry of chord vertices, we get that for odd wheels $\alpha\left(w_{2 h+1}^{u}\right)=0$, and for even wheels $\alpha\left(w_{2 h}^{u}\right)=2 w_{2 h}^{w}$. As a result,

$$
\begin{aligned}
\alpha Z^{u}(K) & =\exp _{\mathcal{A}^{s w}}\left(-\left.\frac{1}{2} \log A(K)\left(e^{h}\right)\right|_{h^{2 n} \rightarrow 2 w_{2 n}}\right) \\
& =\exp _{\mathcal{A}^{s w}}\left(-\left.\log A(K)\left(e^{h}\right)\right|_{h^{2 n} \rightarrow w_{2 n}}\right),
\end{aligned}
$$


which agrees with the formula (22) of Theorem 3.26. Note that since $K$ is 0 -framed, the first part ("sl coded in arrows") of formula (22) is trivial.

\section{Odds and ends}

\subsection{Some dimensions}

The table below lists what we could find about $\mathcal{A}^{v}$ and $\mathcal{A}^{w}$ by crude brute force computations in low degrees. We list degrees 0 through 7 . The spaces we study are $\mathcal{A}^{-}(\uparrow), \mathcal{A}^{s-}(\uparrow)$ (the - in the subscript means " $v$ and $w$ "), and $\mathcal{A}^{r-}(\uparrow)$ which is $\mathcal{A}^{-}(\uparrow)$ moded out by "isolated" arrows, ${ }^{35} \mathcal{P}^{-}(\uparrow)$ which is the space of primitives in $\mathcal{A}^{-}(\uparrow)$, and $\mathcal{A}^{-}(\bigcirc), \mathcal{A}^{s-}(\bigcirc)$, and $\mathcal{A}^{r-}(\bigcirc)$, which are the same as $\mathcal{A}^{-}(\uparrow), \mathcal{A}^{s-}(\uparrow)$, and $\mathcal{A}^{r-}(\uparrow)$ except with closed knots (knots with a circle skeleton) replacing long knots. Each of these spaces we study in three variants: the $\mathrm{v}$ and the $\mathrm{w}$ variants, as well as the usual knots $\mathrm{u}$ variant which is here just for comparison. We also include a row " $\operatorname{dim} \mathcal{G}_{m} \mathcal{L i e}^{-}(\uparrow)$ " for the dimensions of "Lie-algebraic weight systems". Those are explained in the $u$ and $v$ cases in $[6 ; 49 ; 70]$, and in the $w$ case in Section 3.5.

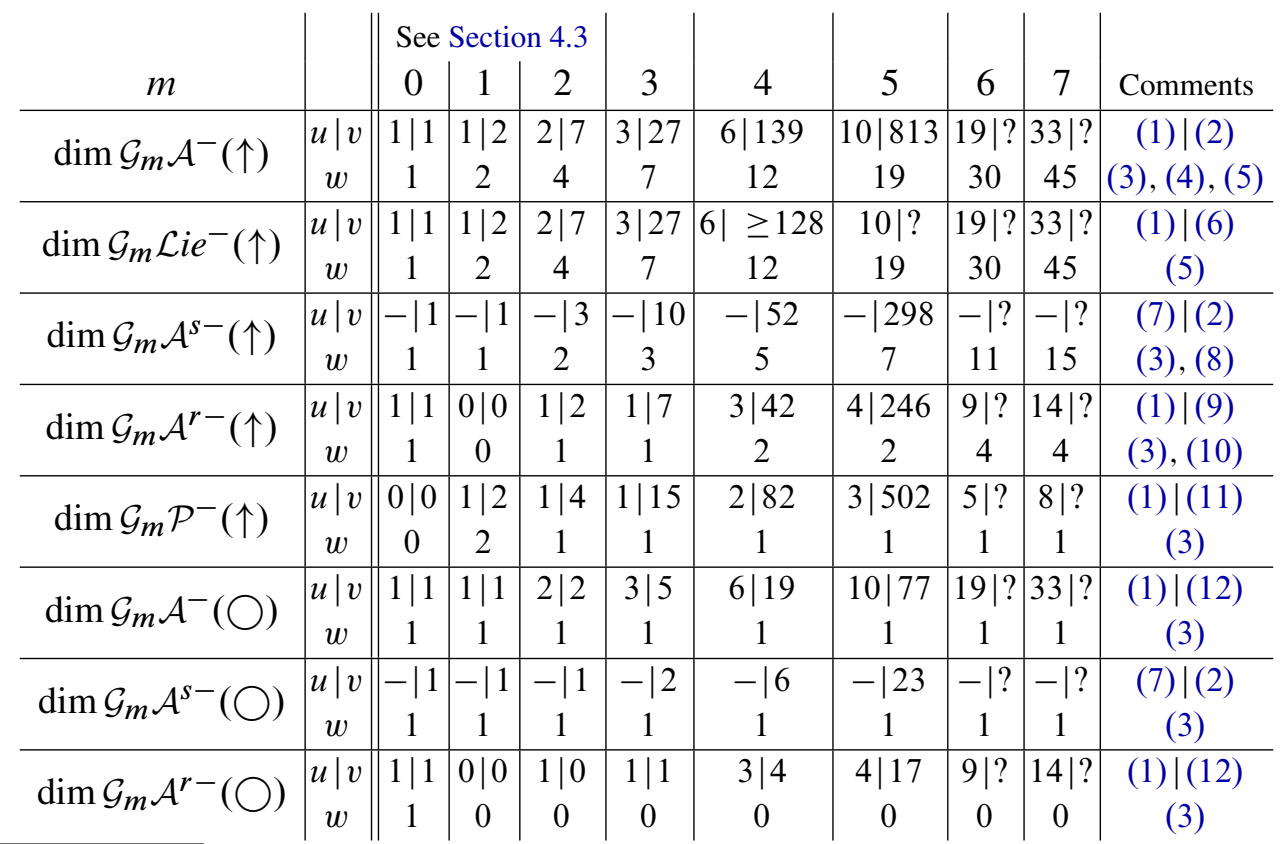

${ }^{35}$ That is, $\mathcal{A}^{r}-(\uparrow)$ is $\mathcal{A}^{-}(\uparrow)$ modulo "framing independence" (FI) relations (see Section 3.4, cf [6], with the isolated arrow taken with either orientation). It is the space related to finite-type invariants of unframed knots, on which the R1 move is also imposed, in the same way as $\mathcal{A}^{-}(\uparrow)$ is related to framed knots. 
Comments 4.1 (1) Much more is known computationally in the u-knots case. See especially $[6 ; 8 ; 56 ; 3]$.

(2) These dimensions were computed by Louis Leung and DBN using a program available at [15, "Dimensions"].

(3) As we have seen in Section 3.4, the spaces associated with w-knots are understood to all degrees.

(4) To degree 4, these numbers were also verified by [15, "Dimensions"].

(5) The next few numbers in these sequences are 67, 97, 139, 195, 272.

(6) These dimensions were computed by Louis Leung and DBN using a program available at [15, "Arrow Diagrams and $\mathfrak{g l}(N)$ "]. Note the match with the row above.

(7) There is no "s" quotient in the "u" case.

(8) The next few numbers in this sequence are 22, 30, 42, 56, 77.

(9) These numbers were computed by [15, "Dimensions"]. Contrary to the $\mathcal{A}^{u}$ case, $\mathcal{A}^{r v}$ is not the quotient of $\mathcal{A}^{v}$ by the ideal generated by degree 1 elements, and therefore the dimensions of the graded pieces of these two spaces cannot be deduced from each other using the Milnor-Moore theorem.

(10) The next few numbers in this sequence are 7, 8, 12, 14, 21.

(11) These dimensions were deduced from the dimensions of $\mathcal{G}_{m} \mathcal{A}^{v}(\uparrow)$ using the Milnor-Moore theorem.

(12) Computed by [15, "Dimensions"]. Contrary to the $\mathcal{A}^{u}$ case, $\mathcal{A}^{v}(\bigcirc), \mathcal{A}^{s v}(\bigcirc)$ and $\mathcal{A}^{r v}(\bigcirc)$ are not isomorphic to $\mathcal{A}^{v}(\uparrow), \mathcal{A}^{s v}(\uparrow)$ and $\mathcal{A}^{r v}(\uparrow)$, and separate computations are required.

\subsection{What do we mean by "closed form"?}

As stated earlier, one of our hopes for this sequence of papers is that it will lead to closed-form formulae for tree-level associators. The notion "closed-form" in itself requires an explanation. Is $e^{x}$ a closed form expression for $\sum_{n=0}^{\infty} x^{n} / n !$, or is it just an artificial name given for a transcendental function we cannot otherwise reduce? Likewise, why not call some tree-level associator $\Phi^{\text {tree }}$ and now it is "in closed form"?

For us, "closed-form" should mean "useful for computations". More precisely, it means that the quantity in question is an element of some space $\mathcal{A}^{c f}$ of "useful closed-form thingies" whose elements have finite descriptions (hopefully, finite and short) and on which some operations are defined by algorithms which terminate in finite time 
(hopefully, finite and short). Furthermore, there should be a finite-time algorithm to decide whether two descriptions of elements of $\mathcal{A}^{c f}$ describe the same element. ${ }^{36} \mathrm{It}$ is even better if the said decision algorithm takes the form "bring each of the two elements in question to a canonical form by means of some finite (and hopefully short) procedure, and then compare the canonical forms verbatim"; if this is the case, then many algorithms that involve managing a large number of elements become simpler and faster.

Thus, for example, polynomials in a variable $x$ are always of closed form, for they are simply described by finite sequences of integers (which in themselves are finite sequences of digits), the standard operations on polynomials $\left(+, \times\right.$, and, say, $\frac{d}{d x}$ ) are algorithmically computable, and it is easy to write the "polynomial equality" computer program. Likewise for rational functions and even for rational functions of $x$ and $e^{x}$.

On the other hand, general elements $\Phi$ of the space $\mathcal{A}^{\text {tree }}\left(\uparrow_{3}\right)$ of potential tree-level associators are not closed-form, for they are determined by infinitely many coefficients. Thus, iterative constructions of associators, such as the one in [10] are computationally useful only within bounded-degree quotients of $\mathcal{A}^{\text {tree }}\left(\uparrow_{3}\right)$ and not as all-degree closedform formulae. Likewise, "explicit" formulae for an associator $\Phi$ in terms of multiple $\zeta$-values (eg [62]) are not useful for computations as it is not clear how to apply tangletheoretic operations to $\Phi$ (such as $\Phi \mapsto \Phi^{1342}$ or $\left.\Phi \mapsto(1 \otimes \Delta \otimes 1) \Phi\right)$ while staying within some space of "objects with finite description in terms of multiple $\zeta$-values". And even if a reasonable space of such objects could be defined, it remains an open problem to decide whether a given rational linear combination of multiple $\zeta$-values is equal to 0 .

\subsection{Arrow diagrams up to degree 2}

Just as an example, in this section we study the spaces $\mathcal{A}^{-}(\uparrow), \mathcal{A}^{s-}(\uparrow), \mathcal{A}^{r-}(\uparrow)$, $\mathcal{P}^{-}(\uparrow), \mathcal{A}^{-}(\bigcirc), \mathcal{A}^{S^{-}}(\bigcirc)$, and $\mathcal{A}^{r-}(\bigcirc)$ in degrees $m \leq 2$ in detail, both in the "v" case and in the "w" case (the "u" case has long been known $[6 ; 56 ; 8]$ ).

4.3.1 Arrow diagrams in degree 0 There is only one degree 0 arrow diagram, the empty diagram $D_{0}$ (see Figure 21). There are no relations, and thus $\left\{D_{0}\right\}$ is the basis of all $\mathcal{G}_{0} \mathcal{A}^{-}(\uparrow)$ spaces, and its closure, the empty circle, is the basis of all $\mathcal{G}_{0} \mathcal{A}^{-}(\bigcirc)$ spaces. $D_{0}$ is the unit 1 , yet $\Delta D_{0}=D_{0} \otimes D_{0}=1 \otimes 1 \neq D_{0} \otimes 1+1 \otimes D_{0}$, so $D_{0}$ is not primitive and $\operatorname{dim} \mathcal{G}_{0} \mathcal{P}^{-}(\uparrow)=0$.

\footnotetext{
${ }^{36}$ In our context, if it is hard to decide within the target space of an invariant whether two elements are equal or not, the invariant is not too useful in deciding whether two knotted objects are equal or not.
} 
4.3.2 Arrow diagrams in degree 1 There are only two degree 1 arrow diagrams, the "right arrow" diagram $D_{R}$ and the "left arrow" diagram $D_{L}$ (see Figure 21). There are no $6 T$ relations, and thus $\left\{D_{R}, D_{L}\right\}$ is the basis of $\mathcal{G}_{1} \mathcal{A}^{-}(\uparrow)$. Modulo RI, $D_{L}=D_{R}$ and hence, $D_{A}:=D_{L}=D_{R}$ is the single basis element of $\mathcal{G}_{1} \mathcal{A}^{S-}(\uparrow)$. Both $D_{R}$ and $D_{L}$ vanish modulo FI, so $\operatorname{dim} \mathcal{G}_{1} \mathcal{A}^{r-}(\uparrow)=\operatorname{dim} \mathcal{G}_{1} \mathcal{A}^{r-}(\bigcirc)=0$. Both $D_{R}$ and $D_{L}$ are primitive, so $\operatorname{dim} \mathcal{G}_{1} \mathcal{P}^{-}(\uparrow)=2$. Finally, the closures $\bar{D}_{R}$ and $\bar{D}_{L}$ of $D_{R}$ and $D_{L}$ are equal, so

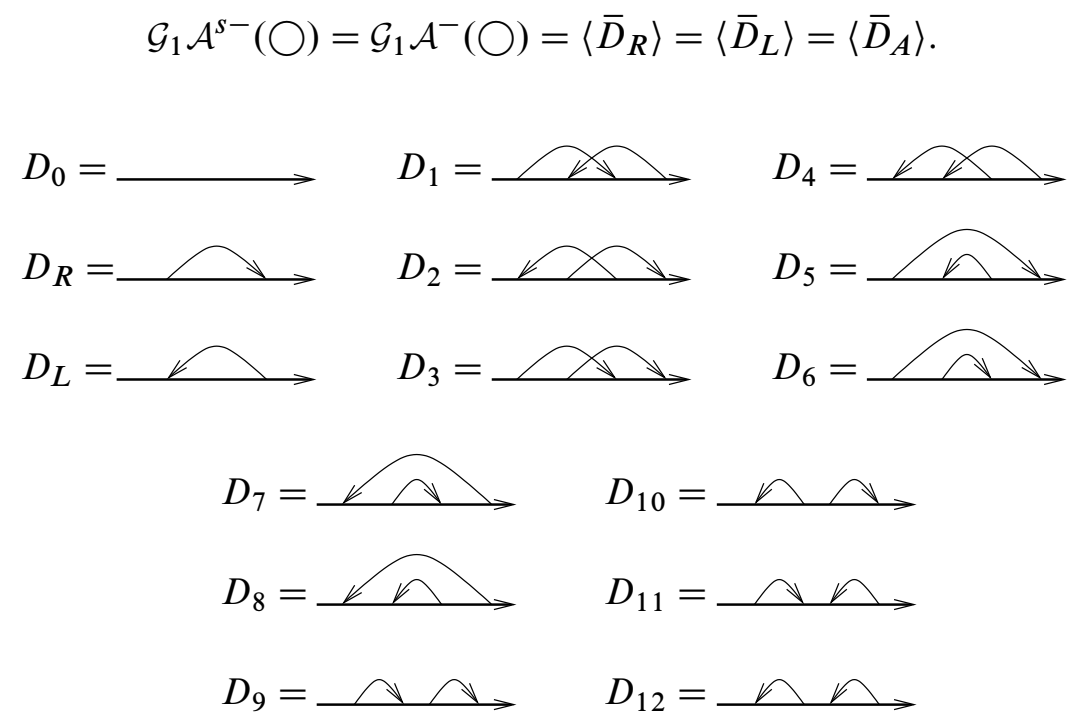

Figure 21: The 15 arrow diagrams of degree at most 2.

4.3.3 Arrow diagrams in degree 2 There are 12 degree 2 arrow diagrams, which we denote $D_{1}, \ldots, D_{12}$ (see Figure 21). There are six $6 T$ relations, corresponding to the 6 ways of ordering the 3 vertical strands that appear in a $6 T$ relation (see Figure 3) along a long line. The ordering $(i j k)$ becomes the relation $D_{3}+D_{9}+D_{3}=D_{6}+D_{3}+D_{6}$. Likewise, $(i k j) \mapsto D_{6}+D_{1}+D_{11}=D_{3}+D_{5}+D_{1},(j i k) \mapsto D_{10}+D_{2}+D_{6}=$ $D_{2}+D_{5}+D_{3},(j k i) \mapsto D_{4}+D_{7}+D_{1}=D_{8}+D_{1}+D_{11},(k i j) \mapsto D_{2}+D_{7}+D_{4}=$ $D_{10}+D_{2}+D_{8}$, and $(k j i) \mapsto D_{8}+D_{4}+D_{8}=D_{4}+D_{12}+D_{4}$. After some linear algebra, we find that $\left\{D_{1}, D_{2}, D_{6}, D_{8}, D_{9}, D_{11}, D_{12}\right\}$ form a basis of $\mathcal{G}_{2} \mathcal{A}^{v}(\uparrow)$, and that the remaining diagrams reduce to the basis as follows: $D_{3}=2 D_{6}-D_{9}$, $D_{4}=2 D_{8}-D_{12}, D_{5}=D_{9}+D_{11}-D_{6}, D_{7}=D_{11}+D_{12}-D_{8}$, and $D_{10}=D_{11}$. In $\mathcal{G}_{2} \mathcal{A}^{s v}(\uparrow)$ we further have that $D_{5}=D_{6}, D_{7}=D_{8}$, and $D_{9}=D_{10}=D_{11}=D_{12}$, and so $\mathcal{G}_{2} \mathcal{A}^{s v}(\uparrow)$ is 3-dimensional with basis $D_{1}, D_{2}$, and $D_{3}=\cdots=D_{12}$. In $\mathcal{G}_{2} \mathcal{A}^{r v}(\uparrow)$ we further have that $D_{5-12}=0$. Thus, $\left\{D_{1}, D_{2}\right\}$ is a basis of $\mathcal{G}_{2} \mathcal{A}^{r v}(\uparrow)$. 
There are 3 OC relations to write for $\mathcal{G}_{2} \mathcal{A}^{w}(\uparrow): D_{2}=D_{10}, D_{3}=D_{6}$, and $D_{4}=D_{8}$. Along with the $6 T$ relations, we find that

$$
\left\{D_{1}, D_{3}=D_{6}=D_{9}, D_{2}=D_{5}=D_{7}=D_{10}=D_{11}, D_{4}=D_{8}=D_{12}\right\}
$$

is a basis of $\mathcal{G}_{2} \mathcal{A}^{w}(\uparrow)$. Similarly $\left\{D_{1}, D_{2}=\cdots=D_{12}\right\}$ is a basis of the twodimensional $\mathcal{G}_{2} \mathcal{A}^{s w}(\uparrow)$. When we mod out by FI, only one diagram remains non-zero in $\mathcal{G}_{2} \mathcal{A}^{r w}(\uparrow)$ and it is $D_{1}$.

We leave the determination of the primitives and the spaces with a circle skeleton as an exercise to the reader.

\section{References}

[1] A Alekseev, B Enriquez, C Torossian, Drinfeld associators, braid groups and explicit solutions of the Kashiwara-Vergne equations, Publ. Math. Inst. Hautes Études Sci. (2010) 143-189 MR2737979

[2] A Alekseev, C Torossian, The Kashiwara-Vergne conjecture and Drinfeld's associators, Ann. of Math. 175 (2012) 415-463 MR2877064

[3] Z Amir-Khosravi, S Sankaran, VasCalc: a Vassiliev invariants calculator Available at http://katlas.math.toronto.edu/drorbn/?title=VasCalc

[4] E Artin, Theory of braids, Ann. of Math. 48 (1947) 101-126 MR0019087

[5] J C Baez, D K Wise, A S Crans, Exotic statistics for strings in 4D BF theory, Adv. Theor. Math. Phys. 11 (2007) 707-749 MR2362007

[6] D Bar-Natan, On the Vassiliev knot invariants, Topology 34 (1995) 423-472 MR1318886

[7] D Bar-Natan, Vassiliev homotopy string link invariants, J. Knot Theory Ramifications 4 (1995) 13-32 MR1321289

[8] D Bar-Natan, Some computations related to Vassiliev invariants (1996) Available at http://www . math . toronto. edu/ drorbn/LOP.html\#Computations

[9] D Bar-Natan, Vassiliev and quantum invariants of braids, from "The interface of knots and physics" (L H Kauffman, editor), Proc. Sympos. Appl. Math. 51, Amer. Math. Soc. (1996) 129-144 MR1372767

[10] D Bar-Natan, Non-associative tangles, from "Geometric topology" (W H Kazez, editor), AMS/IP Stud. Adv. Math. 2, Amer. Math. Soc. (1997) 139-183 MR1470726

[11] D Bar-Natan, On associators and the Grothendieck-Teichmuller group, I, Selecta Math. 4 (1998) 183-212 MR1669949

[12] D Bar-Natan, Algebraic knot theory - a call for action, web document (2006) Available at http://www.math.toronto.edu/ drorbn/papers/AKT-CFA.html 
[13] D Bar-Natan, Finite-type invariants, from "Encyclopedia of Mathematical Physics" (J-P Françoise, G L Naber, T S Tsun, editors), Elsevier, Oxford (2006) 340 - 348

[14] D Bar-Natan, Balloons and hoops and their universal finite-type invariant, BF theory, and an ultimate Alexander invariant, Acta Math. Vietnam. 40 (2015) 271-329 MR3366171

[15] D Bar-Natan, Z Dancso, Finite type invariants of $w$-knotted objects: from Alexander to Kashiwara and Vergne, web version of the first and second papers in one, videos (wClips) and related files Available at http://www.math.toronto.edu/ drorbn/ papers/WKO/

[16] D Bar-Natan, Z Dancso, Finite type invariants of $w$-knotted objects, II: Tangles and the Kashiwara-Vergne problem, preprint (2014) arXiv:1405.1955

[17] D Bar-Natan, S Garoufalidis, L Rozansky, D P Thurston, The Arhus integral of rational homology 3-spheres, I: A highly non trivial flat connection on $S^{3}$, Selecta Math. 8 (2002) 315-339 MR1931167

[18] D Bar-Natan, S Garoufalidis, L Rozansky, D P Thurston, The Arhus integral of rational homology 3-spheres, II: Invariance and universality, Selecta Math. 8 (2002) 341-371 MR1931168

[19] D Bar-Natan, S Garoufalidis, L Rozansky, D P Thurston, The Arhus integral of rational homology 3-spheres, III: Relation with the Le-Murakami-Ohtsuki invariant, Selecta Math. 10 (2004) 305-324 MR2099069

[20] D Bar-Natan, I Halacheva, L Leung, F Roukema, Some dimensions of spaces of finite type invariants of virtual knots, Exp. Math. 20 (2011) 282-287 MR2836253

[21] D Bar-Natan, T T Q Le, D P Thurston, Two applications of elementary knot theory to Lie algebras and Vassiliev invariants, Geom. Topol. 7 (2003) 1-31 MR1988280

[22] D Bar-Natan, S Selmani, Meta-monoids, meta-bicrossed products, and the Alexander polynomial, J. Knot Theory Ramifications 22 (2013) MR3125897

[23] D Bar-Natan, A Stoimenow, The fundamental theorem of Vassiliev invariants, from "Geometry and physics" (J E Andersen, J Dupont, H Pedersen, A Swann, editors), Lecture Notes in Pure and Appl. Math. 184, Dekker, New York (1997) 101-134 MR1423158

[24] V G Bardakov, The virtual and universal braids, Fund. Math. 184 (2004) 1-18 MR2128039

[25] V G Bardakov, P Bellingeri, Combinatorial properties of virtual braids, Topology Appl. 156 (2009) 1071-1082 MR2493369

[26] B Berceanu, Ş Papadima, Universal representations of braid and braid-permutation groups, J. Knot Theory Ramifications 18 (2009) 999-1019 MR2549480

[27] R Bott, C Taubes, On the self-linking of knots, J. Math. Phys. 35 (1994) 5247-5287 MR1295465 
[28] T E Brendle, A Hatcher, Configuration spaces of rings and wickets, Comment. Math. Helv. 88 (2013) 131-162 MR3008915

[29] J S Carter, M Saito, Knotted surfaces and their diagrams, Mathematical Surveys and Monographs 55, Amer. Math. Soc. (1998) MR1487374

[30] A S Cattaneo, P Cotta-Ramusino, J Fröhlich, M Martellini, Topological BF theories in 3 and 4 dimensions, J. Math. Phys. 36 (1995) 6137-6160 MR1355902

[31] A S Cattaneo, P Cotta-Ramusino, M Martellini, Three-dimensional BF theories and the Alexander-Conway invariant of knots, Nuclear Phys. B 436 (1995) 355-382 MR1316372

[32] Z Dancso, On the Kontsevich integral for knotted trivalent graphs, Algebr. Geom. Topol. 10 (2010) 1317-1365 MR2661529

[33] V G Drinfel'd, Quantum groups, from "Proceedings of the International Congress of Mathematicians, I, II” (A M Gleason, editor), Amer. Math. Soc. (1987) 798-820 MR934283

[34] V G Drinfel'd, Quasi-Hopf algebras, Algebra i Analiz 1 (1989) 114-148 MR1047964 In Russian; translated in Leningrad Math. J. 1 (1990) 1419-1457

[35] V G Drinfel'd, On quasitriangular quasi-Hopf algebras and on a group that is closely connected with $\operatorname{Gal}(\overline{\mathbf{Q}} / \mathbf{Q})$, Algebra i Analiz 2 (1990) 149-181 MR1080203 In Russian; translated in Leningrad Math. J. 2 (1991) 829-860

[36] H A Dye, Virtual knots undetected by 1-and 2-strand bracket polynomials, Topology Appl. 153 (2005) 141-160 MR2172041

[37] D B A Epstein, Word processing in groups, A K Peters, Natick, MA (1992)

[38] P Etingof, D Kazhdan, Quantization of Lie bialgebras, I, Selecta Math. 2 (1996) 1-41 MR1403351

[39] R Fenn, R Rimányi, C Rourke, The braid-permutation group, Topology 36 (1997) 123-135 MR1410467

[40] D L Goldsmith, The theory of motion groups, Michigan Math. J. 28 (1981) 3-17 MR600411

[41] M Goussarov, Finite type invariants and n-equivalence of 3-manifolds, C. R. Acad. Sci. Paris Sér. I Math. 329 (1999) 517-522 MR1715131

[42] M Goussarov, M Polyak, O Viro, Finite-type invariants of classical and virtual knots, Topology 39 (2000) 1045-1068 MR1763963

[43] M Gusarov, On n-equivalence of knots and invariants of finite degree, from "Topology of manifolds and varieties" (O Viro, editor), Adv. Soviet Math. 18, Amer. Math. Soc. (1994) 173-192 MR1296895

[44] M Gutiérrez, S Krstić, Normal forms for basis-conjugating automorphisms of a free group, Internat. J. Algebra Comput. 8 (1998) 631-669 MR1682236 
[45] N Habegger, G Masbaum, The Kontsevich integral and Milnor's invariants, Topology 39 (2000) 1253-1289 MR1783857

[46] K Habiro, Claspers and finite type invariants of links, Geom. Topol. 4 (2000) 1-83 MR1735632

[47] K Habiro, T Kanenobu, A Shima, Finite type invariants of ribbon 2-knots, from "Low-dimensional topology" (H Nencka, editor), Contemp. Math. 233, Amer. Math. Soc. (1999) 187-196 MR1701683

[48] K Habiro, A Shima, Finite type invariants of ribbon 2-knots, II, Topology Appl. 111 (2001) 265-287 MR1814229

[49] A Haviv, Towards a diagrammatic analogue of the Reshetikhin-Turaev link invariants, PhD thesis, Hebrew University (2002) arXiv:math.QA/0211031

[50] D Joyce, A classifying invariant of knots, the knot quandle, J. Pure Appl. Algebra 23 (1982) 37-65 MR638121

[51] T Kanenobu, A Shima, Two filtrations of ribbon 2-knots, Topology Appl. 121 (2002) 143-168 MR1903688

[52] M Kashiwara, M Vergne, The Campbell-Hausdorff formula and invariant hyperfunctions, Invent. Math. 47 (1978) 249-272 MR0492078

[53] L H Kauffman, On knots, Annals of Mathematics Studies 115, Princeton Univ. Press (1987) MR907872

[54] L H Kauffman, Virtual knot theory, European J. Combin. 20 (1999) 663-690 MR1721925

[55] L H Kauffman, S Lambropoulou, Virtual braids, Fund. Math. 184 (2004) 159-186 MR2128049

[56] J A Kneissler, The number of primitive Vassiliev invariants up to degree twelve, preprint (1997) arXiv:q-alg/9706022

[57] T Kohno, Vassiliev invariants and de Rham complex on the space of knots, from "Symplectic geometry and quantization" (Y Maeda, H Omori, A Weinstein, editors), Contemp. Math. 179, Amer. Math. Soc. (1994) 123-138 MR1319605

[58] A Kricker, The lines of the Kontsevich integral and Rozansky's rationality conjecture, preprint (2000) arXiv:math/0005284

[59] G Kuperberg, What is a virtual link?, Algebr. Geom. Topol. 3 (2003) 587-591 MR1997331

[60] V Kurlin, Compressed Drinfeld associators, Journal of Algebra 292 (2005) 184-242 MR2166802

[61] T T Q Le, An invariant of integral homology 3-spheres which is universal for all finite type invariants, from "Solitons, geometry, and topology: on the crossroad" (V M Buchstaber, S P Novikov, editors), Amer. Math. Soc. Transl. Ser. 2179 (1997) 75-100 
[62] T Q T Le, J Murakami, Kontsevich's integral for the HOMFLY polynomial and relations between values of multiple zeta functions, Topology Appl. 62 (1995) 193-206 MR1320252

[63] T Q T Le, J Murakami, The universal Vassiliev-Kontsevich invariant for framed oriented links, Compositio Math. 102 (1996) 41-64 MR1394520

[64] T T Q Le, J Murakami, T Ohtsuki, On a universal perturbative invariant of 3manifolds, Topology 37 (1998) 539-574 MR1604883

[65] P Lee, Closed-form associators and braidors in a partly commutative quotient, preprint, University of Toronto (2007) Available at http://individual.utoronto.ca/ PetersKnotPage/FrozenFeet.pdf

[66] P Lee, The pure virtual braid group is quadratic, Selecta Math. 19 (2013) 461-508 MR3090235

[67] P Lee, Proof of a conjectured formula for the Alexander invariant, J. Knot Theory Ramifications 23 (2014) MR3298210

[68] C Lescop, On the cube of the equivariant linking pairing for 3-manifolds of rank one, preprint arXiv:1008.5026

[69] C Lescop, Invariants of knots and 3-manifolds derived from the equivariant linking pairing, from "Chern-Simons gauge theory: 20 years after" (JE Andersen, H U Boden, A Hahn, B Himpel, editors), AMS/IP Stud. Adv. Math. 50, Amer. Math. Soc. (2011) 217-242 MR2809454

[70] L Leung, Combinatorial formulas for classical Lie weight systems on arrow diagrams, preprint, University of Toronto (2008) arXiv:0812.2342

[71] J Lieberum, The Drinfeld associator of gl(1|1), from “Quantum groups” (B Enriquez, editor), IRMA Lect. Math. Theor. Phys. 12, Eur. Math. Soc., Zürich (2008) 39-80 MR2432989

[72] X-S Lin, Power series expansions and invariants of links, from "Geometric topology" (W H Kazez, editor), AMS/IP Stud. Adv. Math. 2, Amer. Math. Soc. (1997) 184-202 MR1470727

[73] W Magnus, A Karrass, D Solitar, Combinatorial group theory: Presentations of groups in terms of generators and relations, Interscience, New York (1966) MR0207802

[74] J McCool, On basis-conjugating automorphisms of free groups, Canad. J. Math. 38 (1986) 1525-1529 MR873421

[75] J W Milnor, J C Moore, On the structure of Hopf algebras, Ann. of Math. 81 (1965) 211-264 MR0174052

[76] G Naot, On Chern-Simons theory with an inhomogeneous gauge group and BF theory knot invariants, J. Math. Phys. 46 (2005) MR2194021 
[77] T Ohtsuki, Finite type invariants of integral homology 3-spheres, J. Knot Theory Ramifications 5 (1996) 101-115 MR1373813

[78] M Polyak, On the algebra of arrow diagrams, Lett. Math. Phys. 51 (2000) 275-291 MR1778064

[79] D Rolfsen, Knots and links, Mathematics Lecture Series 7, Publish or Perish, Houston, TX (1990) MR1277811 Corrected reprint of the 1976 original

[80] F Roukema, Goussarov-Polyak-Viro combinatorial formulas for finite type invariants, preprint (2007) arXiv:0711.4001

[81] S Satoh, Virtual knot presentation of ribbon torus-knots, J. Knot Theory Ramifications 9 (2000) 531-542 MR1758871

[82] D Thurston, Integral expressions for the Vassiliev knot invariants, senior thesis, Harvard University (1995) arXiv:math.QA/9901110

[83] V A Vassiliev, Cohomology of knot spaces, from "Theory of singularities and its applications" (V I Arnol'd, editor), Adv. Soviet Math. 1, Amer. Math. Soc. (1990) 23-69 MR1089670

[84] T Watanabe, Clasper-moves among ribbon 2-knots characterizing their finite type invariants, J. Knot Theory Ramifications 15 (2006) 1163-1199 MR2287439

Department of Mathematics, University of Toronto

Toronto ON M5S 2E4, Canada

Mathematical Sciences Institute, Australian National University

John Dedman Building 27, Union Ln, Canberra ACT 2601, Australia

drorbn@math.toronto.edu, zsuzsanna.dancso@anu.edu.au

http: //www. math.toronto.edu/ drorbn,

http://www.math.toronto.edu/zsuzsi

Received: 12 April 2015 Revised: 1 July 2015 
